\title{
Following the traces *
}

\section{An introduction to conjoint measurement without transitivity and additivity}

\author{
Denis Bouyssou ${ }^{1, *}$ \\ CNRS, LAMSADE, Université Paris Dauphine, Place du Maréchal de Lattre de \\ Tassigny, F-75775 Paris Cedex 16, France. \\ Marc Pirlot ${ }^{2}$ \\ Faculté Polytechnique de Mons, 9, rue de Houdain, B-7000 Mons, Belgium.
}

\begin{abstract}
This paper presents a self-contained introduction to a general conjoint measurement framework for the analysis of nontransitive and/or incomplete binary relations on product sets. It is based on the use of several kinds of marginal traces on coordinates induced by the binary relation.

This framework leads to defining three general families of models depending on the kind of trace that they use. Contrary to most conjoint measurement models, these models do not involve an addition operation. This allows for a simple axiomatic analysis at the cost of very weak uniqueness results.
\end{abstract}

Key words: Multiple criteria analysis, Decision analysis, Measurement theory, Conjoint measurement, Nontransitive preferences

* We wish to thank Roman Słowiński for his invitation to prepare this text syn-
thesizing and consolidating Bouyssou, Pirlot, and Vincke (1997) and Bouyssou and
Pirlot (1999, 2002a,d,f).
* Corresponding author.
Email addresses: bouyssou@lamsade.dauphine.fr (Denis Bouyssou),
marc.pirlot@fpms.ac.be (Marc Pirlot).
1 Part of this work was accomplished while Denis Bouyssou was visiting the Ser-
vice de Mathématique de la Gestion at the Université Libre de Bruxelles (Brussels,
Belgium). The warm hospitality of the Service de Mathématique de la Gestion,
the support of the Belgian Fonds National de la Recherche Scientifique and the
Brussels-Capital Region through a "Research in Brussels" action grant are grate-
fully acknowledged.
2 Part of this work was accomplished while Marc Pirlot was visiting LIP6, Uni- 


\section{Introduction}

Conjoint measurement (Krantz, Luce, Suppes, and Tversky, 1971; Wakker, 1989) studies binary relations defined on product sets. There are many situations in which such binary relations are of central interest. Among them let us mention:

- Multiple criteria decision making using a preference relation comparing alternatives evaluated on several attributes (see Belton and Stewart, 2001; Keeney and Raiffa, 1976; Roy, 1996; von Winterfeldt and Edwards, 1986),

- Decision under uncertainty using a preference relation comparing alternatives evaluated on several states of nature (see Anscombe and Aumann, 1963; Fishburn, 1970b, 1988; Gul, 1992; Savage, 1954; Shapiro, 1979; Wakker, 1984, 1989),

- Consumer theory manipulating preference relations for bundles of several goods (see Debreu, 1959),

- Intertemporal decision making using a preference relation between alternatives evaluated at several moments in time (see Keeney and Raiffa, 1976; Koopmans, 1960, 1972),

- Inequality measurement comparing distributions of wealth across several individuals (see Atkinson, 1970; Ben-Porath and Gilboa, 1994; Ben-Porath, Gilboa, and Schmeidle 1997; Weymark, 1981)

Given a binary relation $\succsim$ on a set $X=X_{1} \times X_{2} \times \cdots \times X_{n}$, the theory of conjoint measurement consists in finding conditions under which it is possible to build a convenient numerical representation of $\succsim$ and to study the uniqueness of this representation. Manipulating numbers is clearly much easier than manipulating binary relations. Furthermore, in most cases, the proofs showing the existence of a numerical representation gives very useful hints on how to build this representation and, thus, assess preferences. Finally, the conditions on $\succsim$ guaranteeing the existence of a numerical representation can be subjected to empirical tests (Krantz et al., 1971). This explains why conjoint measurement has attracted much attention in many different fields of research.

In traditional models of conjoint measurement the binary relation studied is most often supposed to be complete and transitive and the numerical representation is sought to be additive. The central model is the additive utility model such that:

$$
x \succsim y \Leftrightarrow \sum_{i=1}^{n} u_{i}\left(x_{i}\right) \geq \sum_{i=1}^{n} u_{i}\left(y_{i}\right)
$$

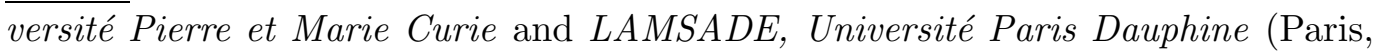
France) thanks to visiting positions in these two institutions and a grant from the Belgian Fonds National de la Recherche Scientifique. Their support is gratefully acknowledged. 
where $u_{i}$ are real-valued functions on the sets $X_{i}$ and it is understood that $x=\left(x_{1}, x_{2}, \ldots, x_{n}\right)$ and $y=\left(y_{1}, y_{2}, \ldots, y_{n}\right)$.

The axiomatic analysis of this model is now quite firmly established (see Debreu, 1960; Krantz et al., 1971; Wakker, 1989) and additive utilities form the basis of many decision analysis techniques (see French, 1993; Keeney and Raiffa, 1976; von Winterfeldt and Edwards, 1986; Wakker, 1989). For recent advances on this model we refer the reader to Fishburn (1992b); Gonzales (1996, 2000, 2003); Karni and Safra (1998); Köbberling (2003); Nakamura (2002).

This central model raises two types of difficulties. First it excludes all relations that would not be complete or transitive whereas the reasonableness of this hypothesis has been challenged by many authors (see May, 1954; Roy, 1996; Tversky, 1969). Second, its axiomatic analysis raises subtle technical difficulties. When $X$ is finite (but of arbitrary cardinality), it is well-known (see Scott, 1964) that its characterization implies using a system of axioms involving a denumerable number of cancellation conditions guaranteeing the existence of solutions to a system of (finitely many) linear inequalities (Jaffray, 1974, extends this approach to sets of arbitray cardinality). Such an axiom system is hardly interpretable and testable. When $X$ is infinite the picture changes provided that conditions are imposed in order to guarantee that the structure of $X$ is "close" to the structure of $\mathbb{R}$ and that $\succsim$ behaves consistently in this continuum. This allows to consider only a limited number of cancellation conditions on $\succsim$. These necessary conditions however interact with the non-necessary structural assumptions imposed on $X$ (e.g. solvability used in Krantz et al. (1971, Chapter 6)), which may obscure their interpretation and test (see Krantz et al. (1971, Chapter 9), Furkhen and Richter (1991)). Furthermore, the analysis of the $n=2$ case has then to be separated from that of the $n \geq 3$ case.

Several authors have forcefully argued in favor of studying conjoint measurement models that would tolerate intransitive and incomplete binary relations (see Bouyssou and Pirlot, 2002d; Fishburn, 1990a, 1991a,b; May, 1954; Roy, 1996; Roy and Bouyssou, 1993; Tversky, 1969). The ones that we propose here will allow us to capture nontransitive and/or incomplete binary relations while being rather simple to analyze from an axiomatic point of view. In order to get a feeling for these various models, it is useful to envisage the various strategies that are likely to be implemented when comparing objects differing on several dimensions (see Dahlstrand and Montgomery, 1984; Montgomery, 1977; Montgomery and Svenson, 1976; Russo and Dosher, 1983; Svenson, 1979; Tversky, 1969).

Consider two alternatives $x$ and $y$ evaluated on a family of $n$ attributes so that $x=\left(x_{1}, x_{2}, \ldots, x_{n}\right)$ and $y=\left(y_{1}, y_{2}, \ldots, y_{n}\right)$. A first strategy that can be used in order to decide whether or not it can be said that " $x$ is at least as 
good as $y$ " consists in trying to measure the "worth" of each alternative on each attribute and then to combine these evaluations adequately. Abandoning all idea of transitivity and completeness, this suggests a model in which:

$$
x \succsim y \Leftrightarrow F\left(u_{1}\left(x_{1}\right), u_{2}\left(x_{2}\right), \ldots, u_{n}\left(x_{n}\right), u_{1}\left(y_{1}\right), u_{2}\left(y_{2}\right), \ldots, u_{n}\left(y_{n}\right)\right) \geq 0
$$

where $u_{i}$ are real-valued functions on the $X_{i}$ and $F$ is a real-valued function on $\prod_{i=1}^{n} u_{i}\left(X_{i}\right)^{2}$. Additional properties on $F$, e.g. its nondecreasingness (resp. nonincreasingness) in its first (resp. last) $n$ arguments, will give rise to a variety of models implementing this first strategy.

A second strategy relies on the idea of measuring "preference differences" separately on each attribute and then combining these (positive or negative) differences in order to know whether the aggregation of these differences leads to an advantage for $x$ over $y$. More formally, this suggests a model in which:

$$
x \succsim y \Leftrightarrow G\left(p_{1}\left(x_{1}, y_{1}\right), p_{2}\left(x_{2}, y_{2}\right), \ldots, p_{n}\left(x_{n}, y_{n}\right)\right) \geq 0
$$

where $p_{i}$ are real-valued functions on $X_{i}^{2}$ and $G$ is a real-valued function on $\prod_{i=1}^{n} p_{i}\left(X_{i}^{2}\right)$. Additional properties on $G$ (e.g. its oddness or its nondecreasingness in each of its arguments) or on $p_{i}$ (e.g. $p_{i}\left(x_{i}, x_{i}\right)=0$ or $p_{i}\left(x_{i}, y_{i}\right)=$ $\left.-p_{i}\left(y_{i}, x_{i}\right)\right)$ will give rise to a variety of models in line with the above strategy.

Of course these two strategies are not incompatible and one may well envisage to use the "worth" of each alternative on each attribute to measure "preference differences". This suggests a model in which:

$x \succsim y \Leftrightarrow H\left(\phi_{1}\left(u_{1}\left(x_{1}\right), u_{1}\left(y_{1}\right)\right), \phi_{2}\left(u_{2}\left(x_{2}\right), u_{2}\left(y_{2}\right)\right), \ldots, \phi_{n}\left(u_{n}\left(x_{n}\right), u_{n}\left(y_{n}\right)\right)\right) \geq 0$

where $u_{i}$ are real-valued functions on $X_{i}, \phi_{i}$ are real-valued functions on $u_{i}\left(X_{i}\right)^{2}$ and $H$ is a real-valued function on $\prod_{i=1}^{n} \phi_{i}\left(u_{i}\left(X_{i}\right)^{2}\right)$.

This paper is devoted to the analysis of several variants of (1.2), (1.3) and (1.4). We shall provide a fairly complete axiomatic analysis of these models and use them as a general framework allowing to understand the characteristics and differences between several aggregation models.

It should be noted that the introduction of intransitivities in conjoint measurement models is not new. A. Tversky (1969) was one of the first to propose such a model generalizing (1.1), known as the additive difference model, in which:

$$
x \succsim y \Leftrightarrow \sum_{i=1}^{n} \Phi_{i}\left(u_{i}\left(x_{i}\right)-u_{i}\left(y_{i}\right)\right) \geq 0
$$

where $\Phi_{i}$ are increasing and odd functions. This model was axiomatized in Fishburn (1992a). More recently, additive nontransitive models were proposed 
in Bouyssou (1986); Fishburn (1990a,b, 1991a, 1992c) and Vind (1991). They are of the following general type:

$$
x \succsim y \Leftrightarrow \sum_{i=1}^{n} p_{i}\left(x_{i}, y_{i}\right) \geq 0
$$

where the $p_{i}$ are real-valued functions on $X_{i}^{2}$ and may have several additional properties (e.g. $p_{i}\left(x_{i}, x_{i}\right)=0$, for all $i \in\{1,2, \ldots, n\}$ and all $x_{i} \in X_{i}$ ).

Comparing (1.2), (1.3) and (1.4) with (1.5) or (1.6) shows that the main particularity of our models lies in the use of a general class of functions instead of just addition and subtraction. This is quite reminiscent of the extension of (1.1) introduced in Krantz et al. (1971, Ch. 7), known as the decomposable transitive model, in which:

$$
x \succsim y \Leftrightarrow F\left(u_{1}\left(x_{1}\right), u_{2}\left(x_{2}\right), \ldots, u_{n}\left(x_{n}\right)\right) \geq F\left(u_{1}\left(y_{1}\right), u_{2}\left(y_{2}\right), \ldots, u_{n}\left(y_{n}\right)\right),
$$

where $F$ is increasing in all its arguments.

The use of very general functional forms, instead of additive ones, will greatly facilitate the axiomatic analysis of our models. It mainly relies on the study of various kinds of traces induced by the preference relation on coordinates and does not require a detailed analysis of tradeoffs between attributes.

The price to pay for such an extension of the scope of conjoint measurement is that the number of parameters that would be needed to assess such models is quite high. Furthermore, none of them is likely to possess any remarkable uniqueness properties. Therefore, although proofs are constructive, these results will not give direct hints on how to devise assessment procedures. The general idea is to use numerical representations as guidelines to understand the consequences of a limited number of cancellation conditions, without imposing any transitivity or completeness requirement on the preference relation and any structural assumptions on the set of objects. They are not studied for their own sake and our results are not intended to provide clues on how to build them.

With few exceptions, this paper does not contain new results but synthesizes results scattered in Bouyssou and Pirlot (1999) and Bouyssou and Pirlot (2002a,d,f); Bouyssou et al. (1997). The presentation of the results is however new, emphasizing the underlying strategy and framework.

We shall suppose throughout the paper that binary relations are defined on countable (i.e. finite or countably infinite) sets, referring the reader to the original papers for the study of the general case. Contrary to what happens with conjoint measurement models involving an addition operation, this restrictive hypothesis will allow us to skip many technical details while maintaining the spirit of the results in the general case. 
This paper is organized as follows. Section 2 presents some background material: we introduce our vocabulary concerning binary relations and recall some well-known facts on traces. Section 3 studies binary relations defined on product sets and introduces various kinds of traces on coordinates. In section 4 we investigate models of type (1.2), using marginal traces on levels. Section 5 studies models of type (1.3) using marginal traces on differences. Section 6 combines these two aspects with the study of models of type (1.4). A final section discusses the results. Examples and technical details are relegated in appendix.

In order to make this paper self-contained, we only omit proofs that are elementary. Therefore, given the variety of models studied, this paper is somewhat long. The reader willing to get a feeling of the results may skip all proofs and remarks without loss of continuity.

\section{Background}

\subsection{Binary relations}

A binary relation $\succsim$ on a set $A$ is a subset of $A \times A$. We write $a \succsim b$ instead of $(a, b) \in \succsim$. A binary relation $\succsim$ on $A$ is said to be:

- reflexive if $[a \succsim a]$,

- complete if $[a \succsim b$ or $b \succsim a]$,

- symmetric if $[a \succsim b] \Rightarrow[b \succsim a]$,

- asymmetric if $[a \succsim b] \Rightarrow \operatorname{Not}[b \succsim a]$,

- transitive if $[a \succsim b$ and $b \succsim c] \Rightarrow[a \succsim c]$,

- Ferrers if

- semi-transitive if

$$
\left.\begin{array}{c}
a \succsim b \\
\text { and } \\
c \succsim d
\end{array}\right\} \Rightarrow\left\{\begin{array}{c}
a \succsim d \\
\text { or } \\
c \succsim b,
\end{array}\right.
$$

$$
\left.\begin{array}{c}
a \succsim b \\
\text { and } \\
b \succsim c
\end{array}\right\} \Rightarrow\left\{\begin{array}{c}
a \succsim d \\
\text { or } \\
d \succsim c,
\end{array}\right.
$$

for all $a, b, c, d \in A$.

The asymmetric (resp. symmetric) part of $\succsim$ is the binary relation $\succ$ (resp. $\sim)$ on $A$ defined letting, for all $a, b \in A, a \succ b \Leftrightarrow[a \succsim b$ and $\operatorname{Not}[b \succsim a]]$ (resp. $a \sim b \Leftrightarrow[a \succsim b$ and $b \succsim a]$ ). A similar convention will hold when $\succsim$ is subscripted and/or superscripted. 
A weak order (resp. an equivalence relation) is a complete and transitive (resp. reflexive, symmetric and transitive) binary relation. An interval order is a complete and Ferrers binary relation; a semiorder is a semi-transitive interval order. If $\succsim$ is an equivalence on $A, A / \succsim$ will denote the set of equivalence classes of $\succsim$ on $A$.

\subsection{Traces of binary relations}

The idea that any binary relation generates various reflexive and transitive binary relations called traces dates back at least to the pioneering work of Luce (1956). The use of traces have proved especially useful in the study of preference structures tolerating intransitive indifference such as semiorders or interval orders (see Aleskerov and Monjardet, 2002; Fishburn, 1985; Pirlot and Vincke, 1997).

Definition 2.1 (Traces of binary relations). Let $\succsim$ be a binary relation on a set $A$. We associate to $\succsim$ three binary relations on $A$, called traces, letting, for all $a, b \in A$ :

Left Trace $a \succsim^{+} b \Leftrightarrow[b \succsim c \Rightarrow a \succsim c]$,

Right Trace $a \succsim^{-} b \Leftrightarrow[c \succsim a \Rightarrow c \succsim b]$,

Trace $a \succsim^{ \pm} b \Leftrightarrow\left[a \succsim^{+} b\right.$ and $\left.a \succsim^{-} b\right]$.

Following our conventions, $\sim^{+}$and $\succ^{+}$will denote the symmetric and asymmetric parts of $\succsim^{+}$, the same holding for $\succsim^{-}$and $\succsim^{ \pm}$. Useful connections between $\succsim$ and its traces are summarized below for the ease of future reference. All of them are straightforward consequences of the preceding definition.

\section{Proposition 2.2 (Properties of traces).}

(1) $\sim^{+}, \sim^{-}$and $\sim^{ \pm}$are equivalence relations (reflexive, symmetric and transitive).

(2) $\succsim^{+}$, $\succsim^{-}$and $\succsim^{ \pm}$are reflexive and transitive binary relations.

(3) For all $a, b, c, d \in A$ :

$$
\begin{aligned}
{\left[a \succsim b, b \succsim^{-} c\right] } & \Rightarrow a \succsim c, \\
{\left[a \succsim b, c \succsim^{+} a\right] } & \Rightarrow c \succsim b, \\
{\left[d \succsim^{ \pm} a, b \succsim^{ \pm} c\right] } & \Rightarrow\left\{\begin{array}{l}
a \succsim b \Rightarrow d \succsim c, \\
a n d \\
a \succ b \Rightarrow d \succsim c,
\end{array}\right. \\
{\left[d \sim^{ \pm} a, b \sim^{ \pm} c\right] } & \Rightarrow\left\{\begin{array}{l}
a \succsim b \Leftrightarrow d \succsim c, \\
a n d \\
a \succ b \Leftrightarrow d \succ c .
\end{array}\right.
\end{aligned}
$$


The following proposition summarizes a number of well-known facts about traces (see Fishburn, 1985; Monjardet, 1978; Pirlot and Vincke, 1997; Roubens and Vincke, 1985).

\section{Proposition 2.3 (Completeness of global traces).}

(1) $\succsim^{+}$is complete $\Leftrightarrow \succsim^{-}$is complete $\Leftrightarrow \succsim$ is Ferrers.

(2) $\succsim^{ \pm}$is complete $\Leftrightarrow \succsim$ is Ferrers and semi-transitive.

For a detailed analysis of the rôle of traces in various domains of preference modelling we refer to Aleskerov and Monjardet (2002); Doignon, Monjardet, Roubens, and Vincke (1988); Laslier (1997); Monjardet (1978); Pirlot and Vincke (1997); Roubens and Vincke (1985).

\subsection{General numerical representations}

We present here very general numerical representations of binary relations that will serve later as guidelines. Although the "results" in this section are part of the folklore of binary relations (see Ebert, 1985), we outline their proof, the logic of which being useful in the sequel.

Let $\succsim$ be a binary relation on a countable (i.e. finite or countably infinite) set $A$. It is clearly always possible to build a, trivial, numerical representation of $\succsim$ such that:

$$
a \succsim b \Leftrightarrow \mathcal{T}(a, b) \geq 0
$$

where $\mathcal{T}$ is a real-valued function on $A^{2}$ defined letting, for all $a, b \in A$ :

$$
\mathcal{T}(a, b)= \begin{cases}+1 & \text { if } a \succsim b \\ -1 & \text { otherwise }\end{cases}
$$

It is possible to even further specify the trivial numerical representation $(\mathcal{T})$ as shown below.

Proposition 2.4 (Trivial numerical representation). Let $\succsim$ be a binary relation on a countable set $A$. There is a real-valued function $u$ on $A$ and $a$ real-valued function $\mathcal{F}$ on $u(A)^{2}$ such that, for all $a, b \in A$ :

$$
a \succsim b \Leftrightarrow \mathcal{F}(u(a), u(b)) \geq 0,
$$

PROOF. The relation $\sim^{ \pm}$is an equivalence. Since $A$ is countable (in fact, as soon as the cardinality of $A / \sim^{ \pm}$is not "too large"), there is a real-valued 
function $u$ on $A$ such that, for all $a, b \in A$ :

$$
a \sim^{ \pm} b \Leftrightarrow u(a)=u(b) .
$$

Take any function $u$ satisfying $(2.5)$ and define $\mathcal{F}$ letting, for all $a, b \in A$ :

$$
\mathcal{F}(u(a), u(b))= \begin{cases}+1 & \text { if } a \succsim b, \\ -1 & \text { otherwise. }\end{cases}
$$

We have to show that $\mathcal{F}$ is well-defined, i.e. that $[u(a)=u(c)$ and $u(b)=u(d)]$ implies $[a \succsim b \Leftrightarrow c \succsim d]$. This follows from (2.4).

Remark 2.5 (Uniqueness). The reader will have noticed that the above proof shows a little more than the mere statement of proposition 2.4. We have in fact shown that there always exists a representation in model $(\mathcal{F} 0)$ in which $u$ represents $\sim^{ \pm}$. This is by no means necessary however. In fact, since $A$ is countable there is a one-to-one function $v$ between $A$ and some countable subset of $\mathbb{R}$. Any such one-to-one function $v$ may clearly be used instead of a function $u$ representing $\sim^{ \pm}$. Since in that case $[v(a)=v(c)$ and $v(b)=v(d)]$ implies $[a=c$ and $b=d]$, using (2.6) always leads to a well-defined function $\mathcal{F}$. This trivial representation is therefore defiantly irregular (see Roberts, 1979, ch. 2). Observe finally that any function $\mathcal{G}$ assigning arbitrary nonnegative values to ordered pairs $(u(a), u(b))$ such that $a \succsim b$ and arbitrary negative values otherwise may be used instead of $\mathcal{F}$.

It is not difficult to extend the above trivial representation to the general uncountable case. All that is required is that the cardinal of $A$ should not be "too large", i.e., there should be enough real numbers to distinguish elements of $A$ that have to be distinguished. This will be the case as soon as there is a one-to-one correspondence between $A / \sim^{ \pm}$and some subset of $\mathbb{R}$.

We now consider nontrivial numerical representations obtained imposing additional constraints on the trivial model $(\mathcal{F} 0)$.

Proposition 2.6 (General models). The function $\mathcal{F}$ in model $(\mathcal{F} 0)$ can be chosen so that, for all $x, y \in X$,

(1) $\mathcal{F}(u(x), u(x)) \geq 0$ iff $\succsim$ is reflexive, $($ model $(\mathcal{F} 1))$.

(2) $\mathcal{F}(u(x), u(y))=-\mathcal{F}(u(y), u(x))$ iff $\succsim$ is complete (model $(\mathcal{F} 2)$ ).

(3) $\mathcal{F}$ is increasing in its first argument and decreasing in its second argument iff $\succsim$ is Ferrers and semi-transitive (model $(\mathcal{F} 3)$ ).

(4) $\mathcal{F}(u(x), u(y))=-\mathcal{F}(u(y), u(x))$ and $\mathcal{F}$ is nondecreasing in its first argument and nonincreasing in its second argument iff $\succsim$ is a semiorder $(\operatorname{model}(\mathcal{F} 4))$.

(5) $\mathcal{F}(u(x), u(y))=-\mathcal{F}(u(y), u(x))$ and $\mathcal{F}$ is increasing in its first argument and decreasing in its second argument iff $\succsim$ is a weak order (model $(\mathcal{F} 5)$ ). 
In this case, it is always possible to take $\mathcal{F}(\alpha, \beta)=\alpha-\beta$ (model $(\mathcal{U} 0))$.

PROOF. Part 1 is obvious in view of proposition 2.4.

Part 2. The necessity of completeness is clear. In order to show sufficiency, take any function $u$ satisfying $(2.5)$ and define $\mathcal{F}$ letting, for all $a, b \in A$ :

$$
\mathcal{F}(u(a), u(b))=\left\{\begin{aligned}
+1 & \text { if } a \succ b \\
0 & \text { if } a \sim b \\
-1 & \text { otherwise }
\end{aligned}\right.
$$

Using the completeness of $\succsim$ and (2.4), it is easy to see that $\mathcal{F}$ is well-defined and skew symmetric.

Part 3. The necessity of Ferrers and semi-transitivity is easily established using the properties of $\mathcal{F}$. Let us, for instance, show that $\succsim$ is semi-transitive. Suppose that $a \succsim b$ and $b \succsim c$. Hence $\mathcal{F}(u(a), u(b)) \geq 0$ and $\mathcal{F}(u(b), u(c)) \geq 0$. If $u(b) \geq u(d)$ then $\mathcal{F}(u(a), u(d)) \geq \mathcal{F}(u(a), u(b)) \geq 0$ so that $a \succsim d$. Otherwise we have $u(d)>u(b)$, which implies $\mathcal{F}(u(d), u(c))>\mathcal{F}(u(b), u(c)) \geq 0$ so that $d \succsim c$.

In order to show sufficiency, remember from part 2 of proposition 2.3 that, when $\succsim$ is Ferrers and semi-transitive, $\succsim^{ \pm}$is a weak order. Since $A$ is countable, there is a real-valued function $u$ such that, for all $a, b \in A$ :

$$
a \succsim^{ \pm} b \Leftrightarrow u(a) \geq u(b) .
$$

Using any function $u$ satisfying (2.8), define $\mathcal{F}$ letting, for all $a, b \in A$,

$$
\mathcal{F}(u(a), u(b))= \begin{cases}+\exp (u(a)-u(b)) & \text { if } a \succsim b, \\ -\exp (u(b)-u(a)) & \text { otherwise. }\end{cases}
$$

That $\mathcal{F}$ is well-defined follows from (2.4). Its monotonicity properties follow from (2.3) and its definition.

Part 4. The necessity of completeness, Ferrers and semi-transitivity is easily established. We show sufficiency. Since $\succsim$ is Ferrers and semi-transitive and $A$ is countable, there is a function $u$ satisfying (2.8). Using any such function $u$, define $\mathcal{F}$ as in (2.7). That $\mathcal{F}$ is well-defined and skew symmetric follows from (2.4), since $\sim^{ \pm}$is the symmetric part of $\succsim^{ \pm}$. The monotonicity properties of $\mathcal{F}$ follow from $(2.3)$.

Part 5. Necessity. Suppose that $a \succsim b$ and $b \succsim c$. Hence $\mathcal{F}(u(a), u(b)) \geq 0$ and $\mathcal{F}(u(b), u(c)) \geq 0$. Since $\mathcal{F}$ is skew symmetric, we know that $\mathcal{F}(u(c), u(b)) \leq 0$. Using the increasingness of $\mathcal{F}, \mathcal{F}(u(a), u(b)) \geq 0$ and $\mathcal{F}(u(c), u(b)) \leq 0$ imply 
$u(a) \geq u(c)$. Since $\mathcal{F}(u(a), u(a))=0$, because $\mathcal{F}$ is skew symmetric, we have $\mathcal{F}(u(a), u(c)) \geq 0$ so that $a \succsim c$. Hence, $\succsim$ is transitive.

Sufficiency. Since $\succsim$ is a weak order and $A$ is countable, there is a function $u$ such that, for all $a, b \in A$ :

$$
a \succsim b \Leftrightarrow u(a) \geq u(b)
$$

Using any such function $u$, define $\mathcal{F}$ letting, for all $a, b \in A, \mathcal{F}(u(a), u(b))=$ $u(a)-u(b)$. The well-definedness of $\mathcal{F}$ easily follows from the fact that $\succsim$ is a weak order.

Remark 2.7 (Uniqueness). A word on the uniqueness of the representations in proposition 2.6 is in order. Observe that in parts 1 and 2, any one-to-one function $v$ between $A$ and some countable subset of $\mathbb{R}$ may be used instead of $u$ representing $\sim^{ \pm}$. The only difference between the two parts being in the flexibility for the choice of $\mathcal{F}$.

For parts 3 and 4, we have shown that $u$ may always be chosen so as to represent $\succsim^{ \pm}$. Again, this is by no means necessary. Any real-valued function $v$ on $A$ such that, for all, $a, b \in A$,

$$
a \succ^{ \pm} b \Rightarrow v(a)>v(b)
$$

may also clearly be used with the same function $\mathcal{F}$ as in the above proof.

For part 5 , the uniqueness depends on whether one wishes a representation in model $(\mathcal{F} 5)$ or in model $(\mathcal{U} 0)$. The uniqueness properties of $u$ in model $(\mathcal{U} 0)$ are well-known. Clearly, model $(\mathcal{F} 5)$ offers much more flexibility. Observe that (2.10) is a necessary condition for $u$ to be used in such a representation. It is no more sufficient however since it might happen that $a \sim^{ \pm} b$ and $a \sim c$. In such a situation, we must guarantee that $u(a)=u(b)$ otherwise the increasingness of $\mathcal{F}$ would lead to $c \succ b$ or $b \succ c$ (depending on whether $u(b)<u(a)$ or $u(b)>u(a))$ whereas $a \sim^{ \pm} b$ and $a \sim c$ imply, using (2.4), $b \sim c$.

In all parts, the possible degrees of freedom in the choice of $\mathcal{F}$ are obvious.

Again, the extension to the general uncountable case should be clear. For parts 1 and 2, it suffices to require that there is a one-to-one correspondence between $A / \sim^{ \pm}$and some subset of $\mathbb{R}$. For all other parts, it is necessary and sufficient to require that the weak order $\succsim^{ \pm}$has a numerical representation (i.e., $A$ must have a countable order dense subset w.r.t. $\succsim^{ \pm}$) since the weak order induced by $u$ refines $\succsim^{ \pm}$.

The numerical representations envisaged so far are summarized in table 1. 
Table 1

General numerical representations

\begin{tabular}{|c|c|c|}
\hline Models & Definition & Conditions \\
\hline$(\mathcal{T})$ & $a \succsim b \Leftrightarrow \mathcal{T}(a, b) \geq 0$ & \\
\hline$\Uparrow$ & & $\varnothing$ \\
\hline$(\mathcal{F} 0)$ & $a \succsim b \Leftrightarrow \mathcal{F}(u(a), u(b)) \geq 0$ & \\
\hline$(\mathcal{F} 1)$ & $(\mathcal{F} 0)$ with $\mathcal{F}(\alpha, \alpha) \geq 0$ & refl. \\
\hline$(\mathcal{F} 2)$ & $(\mathcal{F} 0)$ with $\mathcal{F}(\alpha, \beta)=-\mathcal{F}(\beta, \alpha)$ & cpl. \\
\hline$(\mathcal{F} 3)$ & $(\mathcal{F} 0)$ with $\mathcal{F}(\nearrow \nearrow, \searrow \searrow)$ & $\begin{array}{c}\text { Ferrers and } \\
\text { semi-transitivity }\end{array}$ \\
\hline$(\mathcal{F} 4)$ & $(\mathcal{F} 2)$ with $\mathcal{F}(\nearrow, \searrow)$ & semiorder \\
\hline$(\mathcal{F} 5)$ & $(\mathcal{F} 2)$ with $\mathcal{F}(\nearrow \nearrow, \searrow \searrow)$ & \\
\hline$\Uparrow$ & & weak order \\
\hline$(\mathcal{U} 0)$ & $a \succsim b \Leftrightarrow u(a) \geq u(b)$ & \\
\hline
\end{tabular}

\section{Binary relations on product sets}

In the rest of this paper, we consider a countable set $X=\prod_{i=1}^{n} X_{i}$ with $n \geq 2$. Elements $x, y, z, \ldots$ of $X$ will be interpreted as alternatives evaluated on a set $N=\{1,2, \ldots, n\}$ of attributes. A typical binary relation of $X$ is still denoted as $\succsim$ with $\sim$ and $\succ$ as symmetric and asymmetric parts. Again, a similar convention holds when $\succsim$ is superscripted and/or subscripted. It is useful to interpret $\succsim$ as an "at least as good as" preference relation between multi-attributed alternatives.

For any nonempty subset $J$ of the set of attributes $N$, we denote by $X_{J}$ (resp. $X_{-J}$ ) the set $\prod_{i \in J} X_{i}$ (resp. $\prod_{i \notin J} X_{i}$ ). With customary abuse of notation, $\left(x_{J}, y_{-J}\right)$ will denote the element $w \in X$ such that $w_{i}=x_{i}$ if $i \in J$ and $w_{i}=y_{i}$ otherwise. When $J=\{i\}$ we shall simply write $X_{-i}$ and $\left(x_{i}, y_{-i}\right)$. We say that $\succsim$ is marginally complete for $i \in N$ if $\left(x_{i}, a_{-i}\right) \succsim\left(y_{i}, a_{-i}\right)$ or $\left(y_{i}, a_{-i}\right) \succsim$ $\left(x_{i}, a_{-i}\right)$, for all $x_{i}, y_{i} \in X_{i}$ and all $a_{-i} \in X_{-i}$, i.e. if no incomparability occurs when comparing alternatives differing only on attribute $i \in N$. 


\subsection{Independence and marginal preferences}

In conjoint measurement, one starts with a preference relation $\succsim$ on $X$. It is then important to investigate how this information enables to define preference relations on attributes or subsets of attributes. Let $J \subseteq N$ be a nonempty set of attributes. We define the marginal relation ${ }_{J}$ induced on $X_{J}$ by $\succsim$ letting, for all $x_{J}, y_{J} \in X_{J}$ :

$$
x_{J} \succsim_{J} y_{J} \Leftrightarrow\left(x_{J}, z_{-J}\right) \succsim\left(y_{J}, z_{-J}\right), \text { for all } z_{-J} \in X_{-J},
$$

with asymmetric (resp. symmetric) part $\succ_{J}\left(\right.$ resp. $\left.\sim_{J}\right)$. Note that if $\succsim$ is reflexive (resp. transitive), the same will be true for $\succsim_{J}$. This is clearly not true for completeness however.

We define two other binary relations induced by $\succsim$ on $X_{J}$, letting for all $x_{J}, y_{J} \in X_{J}$,

$$
x_{J} \mathcal{S}_{J} y_{J} \Leftrightarrow\left(x_{J}, z_{-J}\right) \succsim\left(y_{J}, z_{-J}\right), \text { for some } z_{-J} \in X_{-J},
$$

and

$$
x_{J} \mathcal{P}_{J} y_{J} \Leftrightarrow\left(x_{J}, z_{-J}\right) \succ\left(y_{J}, z_{-J}\right) \text {, for some } z_{-J} \in X_{-J} .
$$

Definition 3.1 (Independence and separability). Consider a binary relation $\succsim$ on a set $X=\prod_{i=1}^{n} X_{i}$ and let $J \subseteq N$ be a nonempty subset of attributes. We say that $\succsim$ is:

(1) independent for $J$ if $\mathcal{S}_{J} \subseteq \succsim_{J}$,

(2) separable for $J$ if $\mathcal{P}_{J}$ is asymmetric.

If $\succsim$ is independent (resp. separable) for all nonempty subsets of $N$, we say that $\succsim$ is independent (resp. separable). If $\succsim$ is independent (resp. separable) for all subsets containing a single attribute, we say that $\succsim$ is weakly independent (resp. weakly separable).

Independence is a classical notion in conjoint measurement. It states that common evaluations on some attributes do not influence preference. Whereas independence implies weak independence, it is well-know that the converse is not true (see Wakker, 1989).

Independence implies separability but not vice versa. Separability is a weakening of independence that can be motivated considering aggregation models based on "Max" or "Min". It forbids strict reversals of preference when varying common evaluations on some attribute.

Remark 3.2 (Completeness of $\succsim_{i}$ ). Let us observe that when $\succsim$ is complete and independent for $i \in N$ then $\succsim_{i}$ is clearly complete. It is not difficult 
to see that $\succsim_{i}$ is complete if and only if $\succsim$ is marginally complete and weakly separable for $i \in N$. The marginal preference relation $\succsim_{i}$ reflects the comparison of $x_{i}$ and $y_{i}$ when they are adjoined with the same evaluations on $X_{-i}$. This does not exploit all the information contained in $\succsim$. The marginal traces on levels will do so.

Remark 3.3 (Terminology). Weak independence is called "weak separability" in Wakker (1989). Other authors (see Blackorby, Primont, and Russell, 1978; Färe and Primont, 1981; Mak, 1984) use "separability" instead of independence and "weak separability" instead of separability.

\subsection{Marginal traces on levels}

The definitions and results from section 2.2 clearly apply when $\succsim$ is a binary relation on a product set $X=\prod_{i=1}^{n} X_{i}$. Hence, the binary relation $\succsim$ has a left trace (resp. right trace and trace) $\succsim^{+}$(resp. $\succsim^{-}$and $\succsim^{ \pm}$) that is reflexive and transitive.

Consider an attribute $i \in N$. Sticking to the notation introduced above, $\succsim_{i}^{+}$ (resp. $\succsim_{i}^{-}$and $\succsim_{i}^{ \pm}$) will denote the marginal preference relation induced on $X_{i}$ by $\succsim^{+}$(resp. $\succsim^{-}$and $\succsim^{ \pm}$), i.e.

$$
\begin{aligned}
& x_{i} \succsim_{i}^{+} y_{i} \Leftrightarrow\left[\left(x_{i}, z_{-i}\right) \succsim^{+}\left(y_{i}, z_{-i}\right), \text { for all } z_{-i} \in X_{-i}\right], \\
& x_{i} \succsim_{i}^{-} y_{i} \Leftrightarrow\left[\left(x_{i}, z_{-i}\right) \succsim^{-}\left(y_{i}, z_{-i}\right), \text { for all } z_{-i} \in X_{-i}\right], \\
& x_{i} \succsim_{i}^{ \pm} y_{i} \Leftrightarrow\left[\left(x_{i}, z_{-i}\right) \succsim^{ \pm}\left(y_{i}, z_{-i}\right), \text { for all } z_{-i} \in X_{-i}\right] .
\end{aligned}
$$

Since, by construction, $\succsim^{+}, \succsim^{-}$and $\succsim^{ \pm}$are reflexive and transitive, the same is true for $\succsim_{i}^{+}, \succsim_{i}^{-}$and $\succsim_{i}^{+}$. As shown in the following lemma, $\succsim_{i}^{+}$(resp. $\succsim_{i}^{-}$ and $\succsim_{i}^{ \pm}$), the marginal relation induced on $X_{i}, i \in N$, by the global trace $\succsim^{+}$ (resp. $\succsim_{i}^{-}$and $\succsim_{i}^{ \pm}$) can also be usefully interpreted as a marginal trace on levels on attribute $i \in N$.

Lemma 3.4 (Marginal traces on levels induced by traces). For all $i \in N$, all $x_{i}, y_{i} \in X_{i}$, all $a_{-i} \in X_{-i}$ and all $z \in X$ :

(1) $x_{i} \succsim_{i}^{+} y_{i} \Leftrightarrow\left[\left(y_{i}, a_{-i}\right) \succsim z \Rightarrow\left(x_{i}, a_{-i}\right) \succsim z\right]$,

(2) $x_{i} \succsim_{i}^{-} y_{i} \Leftrightarrow\left[z \succsim\left(x_{i}, a_{-i}\right) \Rightarrow z \succsim\left(y_{i}, a_{-i}\right)\right]$,

(3) $x_{i} \succsim_{i}^{ \pm} y_{i} \Leftrightarrow\left\{\begin{array}{l}\left(y_{i}, a_{-i}\right) \succsim z \Rightarrow\left(x_{i}, a_{-i}\right) \succsim z, \\ \text { and } \\ z \succsim\left(x_{i}, a_{-i}\right) \Rightarrow z \succsim\left(y_{i}, a_{-i}\right) \text {. }\end{array}\right.$

PROOF. We give the proof of part 1, the proof of the other parts being similar. By definition we have: $x_{i} \succsim_{i}^{+} y_{i} \Leftrightarrow\left[\left(x_{i}, a_{-i}\right) \succsim^{+}\left(y_{i}, a_{-i}\right)\right.$, for all 
$\left.a_{-i} \in X_{-i}\right] \Leftrightarrow\left[\left(y_{i}, a_{-i}\right) \succsim z \Rightarrow\left(x_{i}, a_{-i}\right) \succsim z\right.$, for all $a_{-i} \in X_{-i}$ and all $z \in X]$.

Remark 3.5 (Marginal traces on levels and marginal preferences). The above result should clarify the similarities and differences between the marginal traces on levels $\succsim_{i}^{ \pm}$and the marginal preferences $\succsim_{i}$. They are both defined on $X_{i}$. However marginal traces use all information contained in $\succsim$ in order to compare $x_{i}$ with $y_{i}$ including how they compare, when adjoined with the same evaluations on $X_{-i}$, with every other alternatives. On the contrary the definition of $\succsim_{i}$ only relies on the comparison between $x_{i}$ with $y_{i}$ when adjoined with the same evaluations on $X_{-i}$. No other alternative is involved here.

As before, the symmetric and asymmetric parts of $\succsim_{i}^{+}$are respectively denoted $\sim_{i}^{+}$and $\succ_{i}^{+}$, the same convention applying to $\succsim_{i}^{-}$and $\succsim_{i}^{ \pm}$(although it is clearly possible to define marginal traces on a subset of attributes other than singletons, we do not envisage this possibility in this paper).

As in proposition 2.2, there are many interesting connections between marginal traces and $\succsim$. We list some of them in the following lemma, leaving its easy proof as an exercise for the reader.

Lemma 3.6 (Properties of marginal traces on levels). For all $i \in N$ and $x, y, z, w \in X$ :

$$
\begin{gathered}
{\left[x \succsim y, z_{i} \succsim_{i}^{+} x_{i}\right] \Rightarrow\left(z_{i}, x_{-i}\right) \succsim y,} \\
{\left[x \succsim y, y_{i} \succsim_{i}^{-} w_{i}\right] \Rightarrow x \succsim\left(w_{i}, y_{-i}\right),} \\
{\left[z_{i} \succsim_{i}^{ \pm} x_{i}, y_{i} \succsim_{i}^{ \pm} w_{i}\right] \Rightarrow\left\{\begin{array}{l}
x \succsim y \Rightarrow\left(z_{i}, x_{-i}\right) \succsim\left(w_{i}, y_{-i}\right), \\
\text { and } \\
x \succ y \Rightarrow\left(z_{i}, x_{-i}\right) \succ\left(w_{i}, y_{-i}\right),
\end{array}\right.} \\
{\left[z_{i} \sim_{i}^{ \pm} x_{i}, y_{i} \sim_{i}^{ \pm} w_{i}, \text { for all } i \in N\right] \Rightarrow\left\{\begin{array}{l}
x \succsim y \Leftrightarrow z \succsim w, \\
\text { and } \\
x \succ y \Leftrightarrow z \succ w .
\end{array}\right.}
\end{gathered}
$$

It is clear that the marginal traces $\succsim_{i}^{+}, \succsim_{i}^{-}$and $\succsim_{i}^{ \pm}$need not be complete. Interesting consequences will arise when this is the case. This is explored in section 4 .

\subsection{Marginal traces on differences}

Wakker $(1988,1989)$ has powerfully shown how the consideration of induced relations comparing "preference differences" on each attribute may illuminate 
the analysis of conjoint measurement models. We follow the same path in this section.

Given a binary relation $\succsim$ on $X$, we define the binary relation $\succsim_{i}^{*}$ on $X_{i}^{2}$ letting, for all $x_{i}, y_{i}, z_{i}, w_{i} \in X_{i}$,

$$
\begin{aligned}
\left(x_{i}, y_{i}\right) \succsim_{i}^{*}\left(z_{i}, w_{i}\right) \text { iff } & \\
& \quad\left[\text { for all } a_{-i}, b_{-i} \in X_{-i},\left(z_{i}, a_{-i}\right) \succsim\left(w_{i}, b_{-i}\right) \Rightarrow\left(x_{i}, a_{-i}\right) \succsim\left(y_{i}, b_{-i}\right)\right] .
\end{aligned}
$$

Intuitively, if $\left(x_{i}, y_{i}\right) \succsim_{i}^{*}\left(z_{i}, w_{i}\right)$, it seems reasonable to conclude that the preference difference between $x_{i}$ and $y_{i}$ is not smaller than the preference difference between $z_{i}$ and $w_{i}$. Notice that, by construction, $\succsim_{i}^{*}$ is reflexive and transitive.

Contrary to our intuition concerning preference differences, the definition of $\succsim_{i}^{*}$ does not imply that the two "opposite" differences $\left(x_{i}, y_{i}\right)$ and $\left(y_{i}, x_{i}\right)$ are linked. Henceforth we introduce the binary relation $\succsim_{i}^{* *}$ on $X_{i}^{2}$ letting, for all $x_{i}, y_{i}, z_{i}, w_{i} \in X_{i}$,

$$
\left(x_{i}, y_{i}\right) \succsim_{i}^{* *}\left(z_{i}, w_{i}\right) \text { iff }\left[\left(x_{i}, y_{i}\right) \succsim_{i}^{*}\left(z_{i}, w_{i}\right) \text { and }\left(w_{i}, z_{i}\right) \succsim_{i}^{*}\left(y_{i}, x_{i}\right)\right] .
$$

It is easy to see that $\succsim_{i}^{* *}$ is reflexive, transitive and reversible, i.e. $\left(x_{i}, y_{i}\right) \succsim_{i}^{* *}$ $\left(z_{i}, w_{i}\right) \Leftrightarrow\left(w_{i}, z_{i}\right) \succsim_{i}^{* *}\left(y_{i}, x_{i}\right)$.

The relations $\succsim_{i}^{*}$ and $\succsim_{i}^{* *}$ both appear to capture the idea of comparison of preference differences between elements of $X_{i}$ induced by the relation $\succsim$. For the sake of easy reference, we note a few useful connections between $\succsim_{i}^{*}, \succsim_{i}^{* *}$ and $\succsim$ in the following lemma.

Lemma 3.7 (Properties of marginal traces on differences). For all $x, y \in X$ and all $z_{i}, w_{i} \in X_{i}$,

$$
\begin{aligned}
& \succsim \text { is independent iff }\left(x_{i}, x_{i}\right) \sim_{i}^{*}\left(y_{i}, y_{i}\right) \text { for all } i \in N \text {, } \\
& {\left[x \succsim y \text { and }\left(z_{i}, w_{i}\right) \succsim_{i}^{*}\left(x_{i}, y_{i}\right)\right] \Rightarrow\left(z_{i}, x_{-i}\right) \succsim\left(w_{i}, y_{-i}\right) \text {, }} \\
& {\left[\left(z_{i}, w_{i}\right) \sim_{i}^{*}\left(x_{i}, y_{i}\right) \text { for all } i \in N\right] \Rightarrow[x \succsim y \Leftrightarrow z \succsim w] \text {, }} \\
& {\left[x \succ y \text { and }\left(z_{i}, w_{i}\right) \succsim_{i}^{* *}\left(x_{i}, y_{i}\right)\right] \Rightarrow\left(z_{i}, x_{-i}\right) \succ\left(w_{i}, y_{-i}\right) \text {, }} \\
& {\left[\left(z_{i}, w_{i}\right) \sim_{i}^{* *}\left(x_{i}, y_{i}\right) \text { for all } i \in N\right] \Rightarrow\left\{\begin{array}{l}
{[x \succsim y \Leftrightarrow z \succsim w]} \\
\text { and } \\
{[x \succ y \Leftrightarrow z \succ w] .}
\end{array}\right.}
\end{aligned}
$$

PROOF. (3.5). It is clear that $[\succsim$ is independent $] \Leftrightarrow[\succsim$ is independent for $N \backslash\{i\}$, for all $i \in N]$. Observe that $[\succsim$ is independent for $N \backslash\{i\}$, for all $i \in N] \Leftrightarrow\left[\left(x_{i}, a_{-i}\right) \succsim\left(x_{i}, b_{-i}\right) \Leftrightarrow\left(y_{i}, a_{-i}\right) \succsim\left(y_{i}, b_{-i}\right)\right.$, for all $x_{i}, y_{i} \in X_{i}$ and all $\left.a_{-i}, b_{-i} \in X_{-i}\right] \Leftrightarrow\left[\left(x_{i}, x_{i}\right) \sim_{i}^{*}\left(y_{i}, y_{i}\right)\right.$ for all $\left.x_{i}, y_{i} \in X_{i}\right]$. 
(3.6) is obvious from the definition of $\succsim_{i}^{*}$. By induction, (3.7) is immediate.

(3.8). Using (3.6), we know that $\left(z_{i}, x_{-i}\right) \succsim\left(w_{i}, y_{-i}\right)$. Suppose that $\left(w_{i}, y_{-i}\right)$ $\succsim\left(z_{i}, x_{-i}\right)$. Since $\left(z_{i}, w_{i}\right) \succsim_{i}^{* *}\left(x_{i}, y_{i}\right)$ implies $\left(y_{i}, x_{i}\right) \succsim_{i}^{*}\left(w_{i}, z_{i}\right)$, (3.6) implies $y \succsim x$, a contradiction. (3.9) is immediate from (3.8).

It is clear that the marginal traces on differences $\succsim_{i}^{*}$ and $\succsim_{i}^{* *}$ need not be complete. Interesting consequences will arise when this is the case. This is explored in section 5 .

\subsection{Relations between traces on levels and traces on differences}

All the marginal traces envisaged up to this point $\left(\succsim_{i}^{+}, \succsim_{i}^{-}, \succsim_{i}^{ \pm}, \succsim_{i}^{*}\right.$ and $\left.\succsim_{i}^{* *}\right)$ are induced by the same relation $\succsim$. Clearly they are not unrelated. The following lemma shows that they relate exactly as could be expected.

Lemma 3.8 (Relations between marginal traces). For all $i \in N$ and all $x_{i}, y_{i} \in X_{i}$,

(1) $x_{i} \succsim_{i}^{+} y_{i} \Leftrightarrow\left[\left(x_{i}, w_{i}\right) \succsim_{i}^{*}\left(y_{i}, w_{i}\right)\right.$, for all $\left.w_{i} \in X_{i}\right]$,

(2) $x_{i} \succsim_{i}^{-} y_{i} \Leftrightarrow\left[\left(w_{i}, y_{i}\right) \succsim_{i}^{*}\left(w_{i}, x_{i}\right)\right.$, for all $\left.w_{i} \in X_{i}\right]$,

(3) $x_{i} \succsim_{i}^{ \pm} y_{i} \Leftrightarrow\left[\left(x_{i}, w_{i}\right) \succsim_{i}^{* *}\left(y_{i}, w_{i}\right)\right.$, for all $\left.w_{i} \in X_{i}\right]$,

(4) $\left[\ell_{i} \succsim_{i}^{+} x_{i}\right.$ and $\left.\left(x_{i}, y_{i}\right) \succsim_{i}^{*}\left(z_{i}, w_{i}\right)\right] \Rightarrow\left(\ell_{i}, y_{i}\right) \succsim_{i}^{*}\left(z_{i}, w_{i}\right)$,

(5) $\left[y_{i} \succsim_{i}^{-} \ell_{i}\right.$ and $\left.\left(x_{i}, y_{i}\right) \succsim_{i}^{*}\left(z_{i}, w_{i}\right)\right] \Rightarrow\left(x_{i}, \ell_{i}\right) \succsim_{i}^{*}\left(z_{i}, w_{i}\right)$,

(6) $\left[z_{i} \succsim_{i}^{+} \ell_{i}\right.$ and $\left.\left(x_{i}, y_{i}\right) \succsim_{i}^{*}\left(z_{i}, w_{i}\right)\right] \Rightarrow\left(x_{i}, y_{i}\right) \succsim_{i}^{*}\left(\ell_{i}, w_{i}\right)$,

(7) $\left[\ell_{i} \succsim_{i}^{-} w_{i}\right.$ and $\left.\left(x_{i}, y_{i}\right) \succsim_{i}^{*}\left(z_{i}, w_{i}\right)\right] \Rightarrow\left(x_{i}, y_{i}\right) \succsim_{i}^{*}\left(z_{i}, \ell_{i}\right)$,

(8) $\left[x_{i} \sim_{i}^{+} z_{i}\right.$ and $\left.y_{i} \sim_{i}^{-} w_{i}\right] \Rightarrow\left(x_{i}, y_{i}\right) \sim_{i}^{*}\left(z_{i}, w_{i}\right)$,

(9) $\left[x_{i} \sim_{i}^{ \pm} z_{i}\right.$ and $\left.y_{i} \sim_{i}^{ \pm} w_{i}\right] \Rightarrow\left(x_{i}, y_{i}\right) \sim_{i}^{* *}\left(z_{i}, w_{i}\right)$,

PROOF. Part 1. By definition, $\left[x_{i} \succsim_{i}^{+} y_{i}\right] \Leftrightarrow\left[\left(y_{i}, a_{-i}\right) \succsim z \Rightarrow\left(x_{i}, a_{-i}\right) \succsim z\right]$ $\Leftrightarrow\left[\left(x_{i}, z_{i}\right) \succsim_{i}^{*}\left(y_{i}, z_{i}\right)\right.$, for all $\left.z_{i} \in X_{i}\right]$. The proof of part 2 is similar. Part 3 easily follows from parts 1 and 2 .

Part 4. By definition, $\left(x_{i}, y_{i}\right) \succsim_{i}^{*}\left(z_{i}, w_{i}\right)$ iff $\left[\left(z_{i}, a_{-i}\right) \succsim\left(w_{i}, b_{-i}\right) \Rightarrow\left(x_{i}, a_{-i}\right) \succsim\right.$ $\left.\left(y_{i}, b_{-i}\right)\right]$. Since $\ell_{i} \succsim_{i}^{+} x_{i},\left(x_{i}, a_{-i}\right) \succsim\left(y_{i}, b_{-i}\right) \Rightarrow\left(\ell_{i}, a_{-i}\right) \succsim\left(y_{i}, b_{-i}\right)$. Therefore $\left(\ell_{i}, y_{i}\right) \succsim_{i}^{*}\left(z_{i}, w_{i}\right)$. The proof of parts 5,6 and 7 is similar. Part 8 easily follows from parts 4, 5, 6, 7 since $\succsim_{i}^{*}$ is reflexive. Part 9 is immediate from part 8 . 


\subsection{Trivial numerical representations on product sets}

Arbitrary binary relations on product sets have trivial numerical representations of many different kinds (see Bouyssou and Pirlot, 2002a,d,f). We present three of them below that will be easily compared with the general representations introduced above. We often abuse notation in the sequel, writing $\left[u_{i}\left(x_{i}\right)\right]$ instead of $\left(u_{1}\left(x_{1}\right), u_{2}\left(x_{2}\right), \ldots, u_{n}\left(x_{n}\right)\right)$. This should not cause confusion.

Proposition 3.9 (Trivial representations on product sets). Let $\succsim$ be $a$ binary relation on a countable set $X=\prod_{i=1}^{n} X_{i}$.

(1) There are real-valued functions $u_{i}$ on $X_{i}$ and a real valued function $F$ on $\left[\prod_{i=1}^{n} u_{i}\left(X_{i}\right)\right]^{2}$ such that, for all $x, y \in X$,

$$
x \succsim y \Leftrightarrow F\left(\left[u_{i}\left(x_{i}\right)\right] ;\left[u_{i}\left(y_{i}\right)\right]\right) \geq 0 .
$$

(2) There are real-valued functions $p_{i}$ on $X_{i}^{2}$ and a real valued function $G$ on $\prod_{i=1}^{n} p_{i}\left(X_{i}^{2}\right)$ such that, for all $x, y \in X$,

$$
x \succsim y \Leftrightarrow G\left(\left[p_{i}\left(x_{i}, y_{i}\right)\right]\right) \geq 0
$$

(3) There is a real-valued function $u_{i}$ on $X_{i}$, a real-valued function $\phi_{i}$ on $u_{i}\left(X_{i}\right)^{2}$ and a real-valued function $H$ on $\prod_{i=1}^{n} \phi_{i}\left(u_{i}\left(X_{i}\right)^{2}\right)$ such that, for all $x, y \in X$,

$$
x \succsim y \Leftrightarrow H\left(\left[\phi_{i}\left(u_{i}\left(x_{i}\right), u_{i}\left(y_{i}\right)\right)\right]\right) \geq 0
$$

PROOF. Part 1. Let $i \in N$. By construction, $\sim_{i}^{ \pm}$is an equivalence being a reflexive, symmetric and transitive binary relation. Since $X_{i}$ is countable, we know that there is a real-valued function $u_{i}$ on $X_{i}$ such that, for all $x_{i}, y_{i} \in X_{i}$ :

$$
x_{i} \sim_{i}^{ \pm} y_{i} \Leftrightarrow u\left(x_{i}\right)=u_{i}\left(y_{i}\right)
$$

On each $i \in N$, take any function $u_{i}$ satisfying (3.10). Define $F$ on $\left[\prod_{i=1}^{n} u_{i}\left(X_{i}\right)\right]^{2}$ letting:

$$
F\left(\left[u_{i}\left(x_{i}\right)\right] ;\left[u_{i}\left(y_{i}\right)\right]\right)= \begin{cases}+1 & \text { if } x \succsim y \\ -1 & \text { otherwise. }\end{cases}
$$

The well-definedness of $F$ follows from (3.4).

Part 2. Let $i \in N$. By construction, $\sim_{i}^{* *}$ is an equivalence being a reflexive, symmetric and transitive binary relation. Since $X_{i}$ is countable, we know that there is a real-valued function $p_{i}$ on $X_{i}^{2}$ such that, for all $x_{i}, y_{i}, z_{i}, w_{i} \in X_{i}$ :

$$
\left(x_{i}, y_{i}\right) \sim_{i}^{* *}\left(z_{i}, w_{i}\right) \Leftrightarrow p_{i}\left(x_{i}, y_{i}\right)=p_{i}\left(z_{i}, w_{i}\right)
$$


On each $i \in N$, take any function $p_{i}$ satisfying (3.12). Define $G$ on $\prod_{i=1}^{n} p_{i}\left(X_{i}^{2}\right)$ letting:

$$
G\left(\left[p_{i}\left(x_{i}, y_{i}\right)\right]\right)=\left\{\begin{array}{l}
+1 \text { if } x \succsim y, \\
-1 \text { otherwise. }
\end{array}\right.
$$

Using (3.9), it is easy to show that $G$ is well-defined.

Part 3. Take any function $u_{i}$ satisfying (3.10) and any function $p_{i}$ satisfying (3.12). Define $\phi_{i}$ on $u_{i}\left(X_{i}\right)^{2}$ letting, for all $x_{i}, y_{i} \in X_{i}$,

$$
\phi_{i}\left(u_{i}\left(x_{i}\right), u_{i}\left(y_{i}\right)\right)=p_{i}\left(x_{i}, y_{i}\right) .
$$

Let us show that $\phi_{i}$ is well-defined i.e. that $u_{i}\left(x_{i}\right)=u_{i}\left(z_{i}\right)$ and $u_{i}\left(y_{i}\right)=u_{i}\left(w_{i}\right)$ imply $p_{i}\left(x_{i}, y_{i}\right)=p_{i}\left(z_{i}, w_{i}\right)$. By construction, we have $x_{i} \sim_{i}^{ \pm} z_{i}$ and $y_{i} \sim_{i}^{ \pm}$ $w_{i}$ so that, by part 9 of lemma $3.8,\left(x_{i}, y_{i}\right) \sim_{i}^{* *}\left(z_{i}, w_{i}\right)$. Therefore we have $p_{i}\left(x_{i}, y_{i}\right)=p_{i}\left(z_{i}, w_{i}\right)$, as required.

Define $H$ on $\prod_{i=1}^{n} \phi_{i}\left(u_{i}\left(X_{i}\right), u_{i}\left(X_{i}\right)\right)$ letting:

$$
H\left(\left[\phi_{i}\left(u_{i}\left(x_{i}\right), u_{i}\left(y_{i}\right)\right)\right]\right)=\left\{\begin{array}{l}
+1 \text { if } x \succsim y \\
-1 \text { otherwise }
\end{array}\right.
$$

Using (3.7), it is easy to show that $H$ is well-defined.

Remark 3.10 (Uniqueness). As was the case in section 2.3, these various trivial representations are defiantly irregular. A word on uniqueness may clarify things and avoid misunderstandings.

Consider first model $(L 0)$. Since $X_{i}$ is countable, consider any one-to-one correspondence $v_{i}$ between $X_{i}$ and some countable subset of $\mathbb{R}$. Any such correspondence on each attribute may be used instead of the functions $u_{i}$ representing $\sim_{i}^{ \pm}$used in the above proof. Clearly any $F$ assigning arbitrary nonnegative values to $2 n$-tuples $\left(\left[u_{i}\left(x_{i}\right)\right] ;\left[u_{i}\left(y_{i}\right)\right]\right)$ such that $x \succsim y$ and arbitrary negative values otherwise is an acceptable choice.

In model $(D 0)$ any one-to-one function $q_{i}$ between $X_{i}^{2}$ and some countable subset of $\mathbb{R}$ may clearly be used instead of the functions $p_{i}$ representing $\sim_{i}^{* *}$ used in the above proof. Notice that, in the particular case in which, for all $i \in N$ and all $x_{i}, y_{i} \in X_{i},\left(x_{i}, x_{i}\right) \sim_{i}^{* *}\left(y_{i}, y_{i}\right)$, it is always possible to use a skew symmetric function $q_{i}$ (i.e. $q_{i}\left(x_{i}, y_{i}\right)=-q_{i}\left(y_{i}, x_{i}\right)$, for all $x_{i}, y_{i} \in X_{i}$ ) on each attribute. Clearly any function $G$ assigning arbitrary nonnegative values to $n$-tuples $\left[p_{i}\left(x_{i}, y_{i}\right)\right]$ such that $x \succsim y$ and arbitrary negative values otherwise, is an acceptable choice.

If possible, the representation is even more irregular in model (L0D0). Any one-to-one correspondence $v_{i}$ between $X_{i}$ and some countable subset of $\mathbb{R}$ may be used instead of $u_{i}$ representing $\sim_{i}^{ \pm}$used in the above proof. Furthermore 
any one-to-one correspondence $\varphi_{i}$ between $v_{i}\left(X_{i}\right)^{2}$ and some countable subset of $\mathbb{R}$ may be used instead of $\phi_{i}$ representing $\sim_{i}^{* *}$ as done in the above proof. Note, in particular, that it is always possible to choose $\varphi_{i}$ in such a way that it is increasing in its first argument and decreasing in its second argument. Furthermore, as above, if, for all $i \in N$ and all $x_{i}, y_{i} \in X_{i}$, $\left(x_{i}, x_{i}\right) \sim_{i}^{* *}\left(y_{i}, y_{i}\right)$ it is always possible to choose a skew symmetric representation $\varphi_{i}$ (i.e. $\varphi_{i}\left(u_{i}\left(x_{i}\right), u_{i}\left(y_{i}\right)\right)=-\varphi_{i}\left(u_{i}\left(y_{i}\right), u_{i}\left(x_{i}\right)\right)$. Again, any function $H$ assigning arbitrary nonnegative values to $n$-tuples $\left[\phi_{i}\left(u_{i}\left(x_{i}\right), u_{i}\left(y_{i}\right)\right)\right]$ such that $x \succsim y$ and arbitrary negative values otherwise, is an acceptable choice.

Remark 3.11 (Extension to the general case). The above results easily extend to the general uncountable case. In each case, it suffices to require that there are enough real numbers to distinguish the elements that have to be distinguished. This amounts to requiring for model $(D 0)$ that there is a one-toone correspondence between $X_{i}^{2} / \sim_{i}^{* *}$ and some subset of $\mathbb{R}$. For the other two models, the, stronger, requirement that there is a one-to-one correspondence between $X_{i} / \sim_{i}^{ \pm}$and some subset of $\mathbb{R}$ is clearly necessary and sufficient.

We summarize the various trivial models envisaged to this point in table 2 .

Table 2

Trivial numerical representations on product sets

\begin{tabular}{|c|c|}
\hline Models & Definition \\
\hline $\begin{array}{c}(L 0) \\
\Uparrow\end{array}$ & $x \succsim y \Leftrightarrow F\left(\left[u_{i}\left(x_{i}\right)\right] ;\left[u_{i}\left(y_{i}\right)\right]\right) \geq 0$ \\
\hline $\begin{array}{c}(D 0) \\
\mathbb{1}\end{array}$ & $x \succsim y \Leftrightarrow G\left(\left[p_{i}\left(x_{i}, y_{i}\right)\right]\right) \geq 0$ \\
\hline$(L 0 D 0)$ & $x \succsim y \Leftrightarrow H\left(\left[\phi_{i}\left(u_{i}\left(x_{i}\right), u_{i}\left(y_{i}\right)\right)\right]\right) \geq 0$ \\
\hline
\end{tabular}

Our strategy in the sequel is quite similar to the one used in section 2.3. We shall start with each one of the three trivial models $(L 0),(D 0)$ or $(L 0 D 0)$ and study the consequences of imposing additional constraints on these models. We start with model $(L 0)$.

\section{Models using marginal traces on levels ${ }^{3}$}

\subsection{Complete marginal traces on levels}

As was the case with the Ferrers and semi-transitivity conditions when studying traces, we envisage here conditions that will guarantee that marginal traces on levels are complete and, hence, weak orders. As with interval orders and

$\overline{3}$ This section is based on Bouyssou and Pirlot (2002f) 
semiorders, these conditions will prove useful to analyze the underlying structures and to build numerical representations.

Definition 4.1 (Conditions $A C 1, A C 2$ and $A C 3$ ). Let $\succsim$ be a binary relation on a set $X=\prod_{i=1}^{n} X_{i}$. For $i \in N$, this relation is said to satisfy:

$A C 1_{i}$ if

$A C 2_{i}$ if

$$
\left.\begin{array}{c}
x \succsim y \\
\text { and } \\
z \succsim w
\end{array}\right\} \Rightarrow\left\{\begin{array}{l}
\left(z_{i}, x_{-i}\right) \succsim y \\
\text { or } \\
\left(x_{i}, z_{-i}\right) \succsim w
\end{array}\right.
$$

$$
\left.\begin{array}{c}
x \succsim y \\
\text { and } \\
z \succsim w
\end{array}\right\} \Rightarrow\left\{\begin{array}{l}
x \succsim\left(w_{i}, y_{-i}\right) \\
\text { or } \\
z \succsim\left(y_{i}, w_{-i}\right)
\end{array}\right.
$$

$A C 3_{i}$ if

$$
\left.\begin{array}{r}
z \succsim\left(x_{i}, a_{-i}\right) \\
\text { and } \\
\left(x_{i}, b_{-i}\right) \succsim y
\end{array}\right\} \Rightarrow\left\{\begin{array}{l}
z \succsim\left(w_{i}, a_{-i}\right) \\
\text { or } \\
\left(w_{i}, b_{-i}\right) \succsim y
\end{array}\right.
$$

for all $x, y, z, w \in X$, all $a_{-i}, b_{-i} \in X_{-i}$ and all $x_{i}, w_{i} \in X_{i}$.

We say that $\succsim$ satisfies $A C 1$ (resp. $A C 2, A C 3$ ) if it satisfies $A C 1_{i}$ (resp. $A C 2_{i}$, $A C 3_{i}$ ) for all $i \in N$. We sometimes write $A C 123$ as shorthand for $A C 1, A C 2$ and $A C 3$.

These three conditions are transparent variations on the theme of the Ferrers ( $A C 1$ and $A C 2$ ) and semi-transitivity $(A C 3)$ conditions that are made possible by the product structure of $X$. The rationale for the name " $A C$ " is that these conditions are "intrA-attribute Cancellation" conditions.

Condition $A C 1_{i}$ suggests that the elements of $X_{i}$ (instead of the elements of $X$ had the original Ferrers condition been invoked) can be linearly ordered considering "upward dominance": if $x_{i}$ "upward dominates" $z_{i}$ then $\left(z_{i}, c_{-i}\right) \succsim w$ entails $\left(x_{i}, c_{-i}\right) \succsim w$. Condition $A C 2_{i}$ has a similar interpretation considering now "downward dominance". Condition $A C 3_{i}$ ensures that the linear arrangements of the elements of $X_{i}$ obtained considering upward and downward dominance are not incompatible.

Conditions $A C 1, A C 2$ and $A C 3$ were first introduced in Bouyssou and Pirlot (1999); Bouyssou et al. (1997) and later used in Greco, Matarazzo, and Słowiński (2002). The strong links between $A C 1, A C 2, A C 3$ and marginal traces are noted in the following:

Lemma 4.2 (Completeness of marginal traces on levels). We have:

(1) $\succsim_{i}^{+}$is complete iff $A C 1_{i}$ holds, 
(2) $\succsim_{i}^{-}$is complete iff $A C 2_{i}$ holds,

(3) $\left[\operatorname{Not}\left[x_{i} \succsim_{i}^{+} y_{i}\right] \Rightarrow y_{i} \succsim_{i}^{-} x_{i}\right]$ iff $A C 3_{i}$ holds,

(4) $\succsim_{i}^{ \pm}$is complete iff $A C 1_{i}, A C 2_{i}$ and $A C 3_{i}$ hold,

(5) For a binary relation $\succsim$ on $X=\prod_{i=1}^{n} X_{i}$, completeness, $A C 1_{i}, A C 2_{i}$ and $A C 3_{i}$ are independent conditions.

PROOF. Part 1 is proved observing that the negation of $A C 1_{i}$ is equivalent to the negation of the completeness of $\succsim_{i}^{+}$. The proof of part 2 is similar.

Part 3. Suppose that $\operatorname{Not}\left[x_{i} \succsim_{i}^{+} y_{i}\right]$ so that $z \succsim\left(x_{i}, a_{-i}\right)$ and $\operatorname{Not}[z \succsim$ $\left.\left(y_{i}, a_{-i}\right)\right]$, for some $z \in X$ and some $a_{-i} \in X_{-i}$. If $\left(x_{i}, b_{-i}\right) \succsim w$ then $A C 3_{i}$ implies $\left(y_{i}, b_{-i}\right) \succsim w$ or $z \succsim\left(y_{i}, a_{-i}\right)$. Since, by hypothesis, $\operatorname{Not}\left[z \succsim\left(y_{i}, a_{-i}\right)\right]$, we must have $\left(y_{i}, b_{-i}\right) \succsim w$, so that $y_{i} \succsim_{i}^{-} x_{i}$. The reverse implication is proved observing that the negation of $A C 3_{i}$ is equivalent to $\operatorname{Not}\left[y_{i} \succsim_{i}^{+} x_{i}\right]$ and $\operatorname{Not}\left[x_{i} \succsim_{i}^{-} y_{i}\right]$ for some $x_{i}, y_{i} \in X_{i}$. Part 4 immediately results from parts 1,2 and 3.

Part 5: see examples A.1 to A.3 in appendix A.1.

Comparing lemma 4.2 with proposition 2.3 shows an important difference between traces and marginal traces on levels: in the latter case, the right trace may be complete without implying the completeness of the left trace. Hence, the use of three conditions $(A C 1, A C 2$ and $A C 3)$ when studying marginal traces on levels instead of the two classical conditions (Ferrers and semitransitivity) used when studying traces.

The combination of our three conditions $(A C 1, A C 2$ and $A C 3)$ implies that the marginal traces on levels induced by $\succsim$ are weak orders. Unsurprisingly, this implies that marginal relations $\succsim_{i}$ do have special properties even when they differ from marginal traces (which is the general case). We summarize them in the following:

\section{Proposition 4.3 (Properties of marginal preferences).}

(1) If $\succsim$ is reflexive and either $A C 1_{i}$ or $A C 2_{i}$ holds then $\succsim$ is marginally complete and weakly separable for $i \in N$.

(2) If $\succsim$ is reflexive and either $A C 1_{i}$ or $A C 2_{i}$ holds then $\succsim_{i}$ is an interval order.

(3) If, in addition, $\succsim$ satisfies $A C 3_{i}$ then $\succsim_{i}$ is a semiorder.

PROOF. Part 1 . We give the proof using $A C 1_{i}$, the proof using $A C 2_{i}$ being similar. Using the reflexivity of $\succsim$, we know that $\left(x_{i}, a_{-i}\right) \succsim\left(x_{i}, a_{-i}\right)$ and $\left(y_{i}, a_{-i}\right) \succsim\left(y_{i}, a_{-i}\right)$. Since $A C 1_{i}$ holds, $\succsim_{i}^{+}$is complete so that $x_{i} \succsim_{i}^{+} y_{i}$ or 


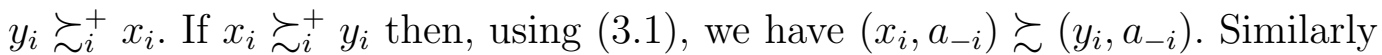
if $y_{i} \succsim_{i}^{+} x_{i}$ then $\left(y_{i}, a_{-i}\right) \succsim\left(x_{i}, a_{-i}\right)$. Hence, $\succsim$ is marginally complete for $i \in N$.

Suppose now that $\succsim$ is not weakly separable for $i \in N$. Then we have $\left(x_{i}, a_{-i}\right) \succ$ $\left(y_{i}, a_{-i}\right)$ and $\left(y_{i}, b_{-i}\right) \succ\left(x_{i}, b_{-i}\right)$, for some $x_{i}, y_{i} \in X_{i}$ and some $a_{-i}, b_{-i} \in X_{-i}$. Since $\succsim$ is reflexive, we have $\left(y_{i}, a_{-i}\right) \succsim\left(y_{i}, a_{-i}\right)$ and $\left(x_{i}, b_{-i}\right) \succsim\left(x_{i}, b_{-i}\right)$. This would imply $\operatorname{Not}\left[x_{i} \succsim_{i}^{+} y_{i}\right]$ and $\operatorname{Not}\left[y_{i} \succsim_{i}^{+} x_{i}\right]$, violating $A C 1_{i}$. Hence, $\succsim$ is weakly separable for $i \in N$.

Part 2 . We know from part 1 that $\succsim$ is marginally complete and weakly separable for $i \in N$. Hence, $\succsim_{i}$ is complete. It remains to prove that $\succsim_{i}$ is Ferrers. Suppose that $x_{i} \succsim_{i} y_{i}$ and $z_{i} \succsim_{i} w_{i}$. Since $A C 1_{i}$ holds, we know that either $x_{i} \succsim_{i}^{+} z_{i}$ or $z_{i} \succsim_{i}^{+} x_{i}$. If $x_{i} \succsim_{i}^{+} z_{i}, z_{i} \succsim_{i} w_{i}$ implies, using the definition of $\succsim_{i}$ and part 1 of lemma 3.4, $x_{i} \succsim_{i} w_{i}$. Similarly if $z_{i} \succsim_{i}^{+} x_{i}, x_{i} \succsim_{i} y_{i}$ implies $z_{i} \succsim_{i} y_{i}$. Hence, $\succsim_{i}$ is Ferrers. The proof using $A C 2_{i}$ is similar.

Part 3. In view of part 2 above, all we have to show is that $\succsim_{i}$ is semi-transitive. Suppose that $x_{i} \succsim_{i} y_{i}$ and $y_{i} \succsim_{i} z_{i}$. Using $A C 1_{i}$, we know that either $w_{i} \succsim_{i}^{+} y_{i}$ or $y_{i} \succ_{i}^{+} w_{i}$. If $w_{i} \succsim_{i}^{+} y_{i}, y_{i} \succsim_{i} z_{i}$ implies, using the definition of $\succsim_{i}$ and part 1 of lemma 3.4, $y_{i} \succsim_{i} z_{i}$. Suppose now that $y_{i} \succ_{i}^{+} w_{i}$. Using $A C 3_{i}$ and part 3 of lemma 4.2, we know that $w_{i} \succsim_{i}^{-} y_{i}$. Using the definition of $\succsim_{i}$ and part 2 of lemma 3.4, $x_{i} \succsim_{i} y_{i}$ and $w_{i} \succsim_{i}^{-} y_{i}$ imply $x_{i} \succsim_{i} w_{i}$. Hence, $\succsim_{i}$ is semi-transitive. The proof using $A C 2_{i}$ is similar.

\subsection{Strict responsiveness to traces on levels}

Keeping in mind the classical constant threshold numerical representation for finite semiorders (see Pirlot and Vincke, 1997; Scott and Suppes, 1958), it is clear that, in general, in a semiorder we may have $x \succsim y, y \succ^{ \pm} z$ and $x \sim z$. Hence, $\succsim$ may not be strictly responsive to $\succ^{ \pm}$even when $\succsim$ and $\succsim^{ \pm}$are complete. Indeed, it is easy to see that a semiorder for which

$$
\left[x \succsim y \text { and } y \succ^{ \pm} z\right] \Rightarrow x \succ z
$$

must be a weak order.

Considering marginal traces on levels, it is now possible to envisage binary relations that are strictly responsive to each of their marginal traces without implying that they are (semi-)transitive or Ferrers.

Definition 4.4 (Conditions $T A C 1, T A C 2$ ). We say that $\succsim$ satisfies: 
$T A C 1_{i}$ if

$$
\left.\begin{array}{l}
\left(x_{i}, a_{-i}\right) \underset{ }{\text { and }} \\
y \succsim\left(z_{i}, a_{-i}\right) \\
\text { and } \\
\left(z_{i}, b_{-i}\right) \succsim w
\end{array}\right\} \Rightarrow\left(x_{i}, b_{-i}\right) \succsim w,
$$

$T A C 2_{i}$ if

$$
\left.\begin{array}{c}
\left(x_{i}, a_{-i}\right) \succsim y \\
\text { and } \\
y \succsim\left(z_{-i}, a_{-i}\right) \\
\text { and } \\
w \succsim\left(x_{i}, b_{-i}\right)
\end{array}\right\} \Rightarrow w \succsim\left(z_{i}, b_{-i}\right),
$$

for all $x_{i}, z_{i} \in X_{i}$, all $a_{-i}, b_{-i} \in X_{-i}$ and all $y, w \in X$.

We say that $\succsim$ satisfies $T A C 1$ (resp. TAC2) if it satisfies $T A C 1_{i}\left(\right.$ resp. $T A C 2_{i}$ ) for all $i \in N$. We sometimes write TAC12 instead of TAC1 and TAC2.

The first two conditions in the premise of $T A C 1_{i}$ and $T A C 2_{i}$ (the rationale for the names being that TAC1 and TAC2 are "intrA-attribute Cancellation" involving Three conditions in their premise) suggest that the level $x_{i}$ is not worse than the level $z_{i}$. TAC $1_{i}$ (resp. TAC $2_{i}$ ) then implies than $x_{i}$ should upward dominate (resp. downward dominate) $z_{i}$.

Lemma 4.5 (Strict responsiveness to marginal traces on levels). If is a complete binary relation on $X=\prod_{i=1}^{n} X_{i}$ then:

(1) $T A C 1_{i} \Rightarrow\left[A C 1_{i}\right.$ and $\left.A C 3_{i}\right]$

(2) $T A C 2_{i} \Rightarrow\left[A C 2_{i}\right.$ and $\left.A C 3_{i}\right]$

(3) $\mathrm{TAC} 1_{i}$ is equivalent to the completeness of $\succsim_{i}^{+}$and the following condition:

$$
\left[x \succsim y \text { and } z_{i} \succ_{i}^{+} x_{i}\right] \Rightarrow\left(z_{i}, x_{-i}\right) \succ y \text {. }
$$

(4) $T A C 2_{i}$ is equivalent to the completeness of $\succsim_{i}^{-}$and the following condition:

$$
\left[x \succsim y \text { and } y_{i} \succ_{i}^{-} w_{i}\right] \Rightarrow x \succ\left(w_{i}, y_{-i}\right) .
$$

(5) If $T A C 1_{i}$ or $T A C 2_{i}$ hold then $\succsim$ is independent for $\{i\}$ and $\succsim_{i}$ is a weakorder. Furthermore when TAC12 $2_{i}$ holds then $\succsim_{i}=\succsim_{i}^{ \pm}$.

(6) In the class of complete relations, TAC1 and TAC2 are independent conditions.

(7) There are weakly independent semiorders verifying TAC1 and TAC2 that are not weak orders.

PROOF. Part 1. Let us first show that when $\succsim$ is complete, $T A C 1_{i} \Rightarrow$ $A C 1_{i}$. Suppose that $A C 1_{i}$ is violated so that $\left(x_{i}, a_{-i}\right) \succsim y,\left(z_{i}, b_{-i}\right) \succsim w$, 
$\operatorname{Not}\left[\left(z_{i}, a_{-i}\right) \succsim y\right]$ and $\operatorname{Not}\left[\left(x_{i}, b_{-i}\right) \succsim w\right]$. Since $\succsim$ is complete, we know that $y \succsim\left(z_{i}, a_{-i}\right)$. Using $T A C 1_{i},\left(x_{i}, a_{-i}\right) \succsim y, y \succsim\left(z_{i}, a_{-i}\right)$ and $\left(z_{i}, b_{-i}\right) \succsim w$ imply $\left(x_{i}, b_{-i}\right) \succsim w$, a contradiction. Hence $A C 1_{i}$ holds and $\succsim_{i}^{+}$is complete.

Similarly suppose that $A C 3_{i}$ is violated so that $z \succsim\left(x_{i}, a_{-i}\right),\left(x_{i}, b_{-i}\right) \succsim y$, $\operatorname{Not}\left[z \succsim\left(w_{i}, a_{-i}\right)\right]$ and $\operatorname{Not}\left[\left(w_{i}, b_{-i}\right) \succsim y\right]$. Since $\succsim$ is complete, we know that $\left(w_{i}, a_{-i}\right) \succsim z$. Using TAC $1_{i},\left(w_{i}, a_{-i}\right) \succsim z, z \succsim\left(x_{i}, a_{-i}\right)$ and $\left(x_{i}, b_{-i}\right) \succsim y$ imply $\left(w_{i}, b_{-i}\right) \succsim y$, a contradiction. The proof of part 2 is similar.

Part 3. $[\Rightarrow]$. We know from part 1 that $A C 1_{i}$ holds so that $\succsim_{i}^{+}$is complete. Suppose now, in contradiction with (4.2) that $x \succsim y, z_{i} \succ_{i}^{+} x_{i}$ and $y \succsim$ $\left(z_{i}, x_{-i}\right)$. We know that $\operatorname{Not}\left[x_{i} \succsim_{i}^{+} z_{i}\right]$, so that $\left(z_{i}, a_{-i}\right) \succsim w$ and $w \succ\left(x_{i}, a_{-i}\right)$, for some $w \in X$ and some $a_{-i} \in X_{-i}$. Using TAC1 $1_{i}, x \succsim y, y \succsim\left(z_{i}, x_{-i}\right)$ and $\left(z_{i}, a_{-i}\right) \succsim w$ imply $\left(x_{i}, a_{-i}\right) \succsim w$, a contradiction.

$[\Leftarrow]$. Suppose that $T A C 1_{i}$ is violated so that $\left(x_{i}, a_{-i}\right) \succsim y, y \succsim\left(z_{i}, a_{-i}\right)$ $\left(z_{i}, b_{-i}\right) \succsim w$ and $w \succ\left(x_{i}, b_{-i}\right)$. This implies $\operatorname{Not}\left[x_{i} \succsim_{i}^{+} z_{i}\right]$. Since $\succsim_{i}^{+}$is complete, we have $z_{i} \succ_{i}^{+} x_{i}$. Using (4.2), $\left(x_{i}, a_{-i}\right) \succsim y$ and $z_{i} \succ_{i}^{+} x_{i}$ would imply $\left(z_{i}, a_{-i}\right) \succ y$, a contradiction.

The proof of part 4 is similar.

Part 5. We give the proof using $T A C 1_{i}$, the proof using $T A C 2_{i}$ being similar. Suppose that $\left(x_{i}, a_{-i}\right) \succsim\left(y_{i}, a_{-i}\right)$ and $\operatorname{Not}\left[\left(x_{i}, b_{-i}\right) \succsim\left(y_{i}, b_{-i}\right)\right]$, for some $x_{i}, y_{i} \in X_{i}$ and some $a_{-i}, b_{-i} \in X_{-i}$. Since $\succsim$ is complete, we know that $\left(y_{i}, b_{-i}\right) \succsim\left(y_{i}, b_{-i}\right)$. Thus, since we know from part 1 that $\succsim_{i}^{+}$is complete, we have $y_{i} \succ_{i}^{+} x_{i}$. Using (4.2), $y_{i} \succ_{i}^{+} x_{i}$ and $\left(x_{i}, a_{-i}\right) \succsim\left(y_{i}, a_{-i}\right)$ would imply $\left(y_{i}, a_{-i}\right) \succ\left(y_{i}, a_{-i}\right)$, a contradiction. Hence, $\succsim$ is independent for $\{i\}$.

Using the reflexivity of $\succsim$, and (3.1), we have: $x_{i} \succsim_{i}^{+} y_{i} \Rightarrow x_{i} \succsim_{i} y_{i}$. Let us show that $x_{i} \succ_{i}^{+} y_{i} \Rightarrow x_{i} \succ_{i} y_{i}$, which will complete the proof since $\succsim_{i}^{+}$is complete. Suppose that $x_{i} \succ_{i}^{+} y_{i}$. Since $\succsim$ is reflexive, we have $\left(y_{i}, a_{-i}\right) \succsim\left(y_{i}, a_{-i}\right)$, for all $a_{-i} \in X_{-i}$. Using (4.2), we obtain $\left(x_{i}, a_{-i}\right) \succ\left(y_{i}, a_{-i}\right)$, for all $a_{-i} \in X_{-i}$. We thus have $x_{i} \succ_{i} y_{i}$. Note that when both $T A C 1_{i}$ and $T A C 2_{i}$ hold, the above reasoning shows that $\succsim_{i}^{ \pm}=\succsim_{i}$.

Parts 6 and 7: see examples A.4 and A.5 in appendix A.1.

As soon as $\succsim$ is complete, the conjunction of $T A C 1_{i}$ and $T A C 2_{i}$ is therefore exactly what is needed to ensure the strict responsiveness of $\succsim$ with respect to $\succ_{i}^{ \pm}$. It also implies that $\succsim$ is independent for $\{i\}$ and that $\succsim_{i}$ is a weak order that is identical to $\succsim_{i}^{ \pm}$. It does not imply however that $\succsim$ is (semi-)transitive or Ferrers. Using (4.2) and (4.3) will facilitate the test of $T A C 1_{i}$ and $T A C 2_{i}$. 


\subsection{Marginal traces on levels and numerical representations}

\subsubsection{Marginal traces and numerical representations}

In model $(L 0)$, the rôle of $u_{i}$ is merely to attach a number to each equivalence class of $X_{i} / \sim_{i}^{ \pm}$while $F$ passively recodes as +1 's and -1 's the presence or absence of $\succsim$ for every possible combination of elements of $X_{i} / \sim_{i}^{ \pm}$. Clearly, as was the case in section 2.3, the situation changes as soon as additional properties are imposed on $F$.

Starting from the trivial model $(L 0)$ in which:

$$
x \succsim y \Leftrightarrow F\left(\left[u_{i}\left(x_{i}\right)\right] ;\left[u_{i}\left(y_{i}\right)\right]\right) \geq 0,
$$

we envisage:

- $(L 1)$ adding to $(L 0)$ the fact that $F\left(\left[u_{i}\left(x_{i}\right)\right] ;\left[u_{i}\left(x_{i}\right)\right]\right) \geq 0$,

- (L2) adding to $(L 1)$ the fact that $F\left(\left[u_{i}\left(x_{i}\right)\right] ;\left[u_{i}\left(y_{i}\right)\right]\right)=-F\left(\left[u_{i}\left(y_{i}\right)\right] ;\left[u_{i}\left(x_{i}\right)\right]\right)$.

Furthermore, in each of $(L 0),(L 1)$ and $(L 2)$, we envisage here the consequences of supposing that $F$ is nondecreasing (resp. increasing) in its first $n$ arguments and nonincreasing (resp. decreasing) in its last $n$ arguments. These eight models are defined in table 3 .

Table 3

Models involving traces on levels

$$
\begin{gathered}
x \succsim y \Leftrightarrow F\left(\left[u_{i}\left(x_{i}\right)\right] ;\left[u_{i}\left(y_{i}\right)\right]\right) \geq 0 \\
(L 0) \text { with } F\left(\left[u_{i}\left(x_{i}\right)\right] ;\left[u_{i}\left(x_{i}\right)\right]\right) \geq 0
\end{gathered}
$$$$
\text { (L0) with } F\left(\left[u_{i}\left(x_{i}\right)\right] ;\left[u_{i}\left(y_{i}\right)\right]\right)=-F\left(\left[u_{i}\left(y_{i}\right)\right] ;\left[u_{i}\left(x_{i}\right)\right]\right)
$$

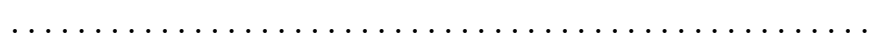

(L0) with $F$ nondecreasing, nonincreasing,

$$
(L 0) \text { with } F \text { increasing, decreasing, }
$$

$(L 1)$ with $F$ nondecreasing, nonincreasing,

$$
(L 1) \text { with } F \text { increasing, decreasing, }
$$

(L2) with $F$ nondecreasing, nonincreasing, (L2) with $F$ increasing, decreasing,

The implications between these various models are clear from their definitions. They are summarized in figure 1 .

\section{Lemma 4.6 (Necessary conditions).}

(1) Model (L1) implies that $\succsim$ is reflexive,

(2) Model (L2) implies that $\succsim$ is complete, 
Figure 1. Implication between models involving traces on levels

$$
\begin{array}{cccccc}
(L 8) & \Rightarrow & (L 6) & \Rightarrow & (L 4) & (\nearrow \nearrow, \searrow \searrow \\
\Downarrow & & \Downarrow & & \Downarrow & \\
(L 7) & \Rightarrow & (L 5) & \Rightarrow & (L 3) & (\nearrow, \searrow) \\
\Downarrow & & \Downarrow & & \Downarrow & \\
(L 2) & \Rightarrow & (L 1) & \Rightarrow & (L 0) & \varnothing \\
& & & & & \\
F(\alpha, \beta)= & & F(\alpha, \alpha) \geq 0 & & \varnothing & \\
-F(\beta, \alpha) & & & & &
\end{array}
$$

$(\nearrow \nearrow, \searrow)$ : increasing (resp. decreasing) in first (resp. last) $n$ arguments $(\nearrow, \searrow)$ : nondecreasing (resp. nonincreasing) in first (resp. last) $n$ arguments

(3) Model (L3) implies AC1, AC2, AC3,

(4) Model (L8) implies TAC1 and TAC2.

PROOF. Parts 1 and 2 are obvious.

Part 3. The fact that $A C 1, A C 2$ and $A C 3$ hold is easily shown using the properties of $F$. We take the case of $A C 3$. Suppose that $\left(x_{i}, a_{-i}\right) \succsim y$ and $w \succsim\left(x_{i}, b_{-i}\right)$ so that, abusing notation, $F\left(\left[u_{i}\left(x_{i}\right),\left(u_{j}\left(a_{j}\right)\right)_{j \neq i}\right] ;\left[u_{i}\left(y_{i}\right)\right]\right) \geq 0$ and $F\left(\left[u_{i}\left(w_{i}\right)\right] ;\left[u_{i}\left(x_{i}\right),\left(u_{j}\left(b_{j}\right)_{j \neq i}\right)\right]\right) \geq 0$.

If $u_{i}\left(z_{i}\right)>u_{i}\left(x_{i}\right)$ then $F\left(\left[u_{i}\left(z_{i}\right),\left(u_{j}\left(a_{j}\right)_{j \neq i}\right)\right] ;\left[u_{i}\left(y_{i}\right)\right]\right) \geq 0$ so that $\left(z_{i}, a_{-i}\right) \succsim y$. Otherwise $u_{i}\left(x_{i}\right) \geq u_{i}\left(z_{i}\right)$ leads to $F\left(\left[u_{i}\left(w_{i}\right)\right] ;\left[u_{i}\left(z_{i}\right),\left(u_{j}\left(b_{j}\right)_{j \neq i}\right)\right]\right) \geq 0$ so that $w \succsim\left(z_{i}, b_{-i}\right)$.

Part 4. Suppose that $\left(x_{i}, a_{-i}\right) \succsim y, y \succsim\left(z_{i}, a_{-i}\right),\left(z_{i}, b_{-i}\right) \succsim w$ and $\operatorname{Not}\left[\left(x_{i}, b_{-i}\right) \succsim\right.$ $w]$. Using the increasingness of $F$ in its first $n$ arguments, the last two conditions imply that $u_{i}\left(z_{i}\right)>u_{i}\left(x_{i}\right)$. But $\left(x_{i}, a_{-i}\right) \succsim y$ and $u_{i}\left(z_{i}\right)>u_{i}\left(x_{i}\right)$ imply $\left(z_{i}, a_{-i}\right) \succ y$, a contradiction. Hence the necessity of TAC1. The necessity is TAC2 is proved similarly.

We are now in position to characterize all our models involving traces on levels.

Theorem 4.7 (Models using traces on levels). Let $\succsim$ be a binary relation on a countable set $X=\prod_{i=1}^{n} X_{i}$.

(1) Model (L1) holds iff $\succsim$ is reflexive.

(2) Model (L2) holds iff $\succsim$ is complete.

(3) Model (L4) holds iff $\succsim$ satisfies $A C 1, A C 2$ and $A C 3$.

(4) Model (L6) holds iff $\succsim$ is reflexive and satisfies $A C 1, A C 2$ and $A C 3$.

(5) Model (L7) holds iff $\succsim$ is complete and satisfies AC1, AC2 and AC3.

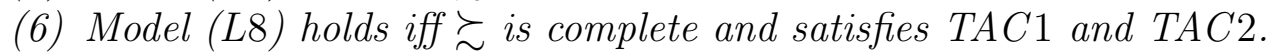


PROOF. For all parts, necessity results from lemma 4.6 and the implications between our models. We show sufficiency.

Part 1 is immediate from part 1 of proposition 3.9.

Part 2. Take, on each $i \in N$, a function $u_{i}$ satisfying (3.10) and define $F$ on $\left[\prod_{i=1}^{n} u_{i}\left(X_{i}\right)\right]^{2}$ letting:

$$
F\left(\left[u_{i}\left(x_{i}\right)\right] ;\left[u_{i}\left(y_{i}\right)\right]\right)=\left\{\begin{aligned}
+1 & \text { if } x \succ y \\
0 & \text { if } x \sim y \\
-1 & \text { otherwise }
\end{aligned}\right.
$$

The well-definedness of $F$ follows from (3.4).

Part 3. Since $A C 1, A C 2$ and $A C 3$ hold, we know from part 4 of lemma 4.2 that $\succsim_{i}^{ \pm}$is a weak order. Since $X_{i}$ is countable, there is a real-valued function $u_{i}$ on $X_{i}$ such that, for all $x_{i}, y_{i} \in X_{i}$ :

$$
x_{i} \succsim_{i}^{ \pm} y_{i} \Leftrightarrow u_{i}\left(x_{i}\right) \geq u_{i}\left(y_{i}\right)
$$

Take, on each $i \in N$, a function $u_{i}$ satisfying (4.5) and define $F$ on $\left[\prod_{i=1}^{n} u_{i}\left(X_{i}\right)\right]^{2}$ letting:

$$
F\left(\left[u_{i}\left(x_{i}\right)\right] ;\left[u_{i}\left(y_{i}\right)\right]= \begin{cases}+\exp \left(\sum_{i=1}^{n}\left(u_{i}\left(x_{i}\right)-u_{i}\left(y_{i}\right)\right)\right) & \text { if } x \succsim y, \\ -\exp \left(\sum_{i=1}^{n}\left(u_{i}\left(y_{i}\right)-u_{i}\left(x_{i}\right)\right)\right) & \text { otherwise. }\end{cases}\right.
$$

The well-definedness of $F$ results from (3.4). The monotonicity properties of $F$ follow from (3.3) and its definition. The proof of part 4 is immediate from part 3.

Part 5. Take, on each $i \in N$, a function $u_{i}$ satisfying (4.5) and define $F$ on $\left[\prod_{i=1}^{n} u_{i}\left(X_{i}\right)\right]^{2}$ letting:

$$
F\left(\left[u_{i}\left(x_{i}\right)\right] ;\left[u_{i}\left(y_{i}\right)\right]\right)= \begin{cases}+\exp \left(\sum_{i=1}^{n}\left(u_{i}\left(x_{i}\right)-u_{i}\left(y_{i}\right)\right)\right) & \text { if } x \succ y \\ 0 & \text { if } x \sim y \\ -\exp \left(\sum_{i=1}^{n}\left(u_{i}\left(y_{i}\right)-u_{i}\left(x_{i}\right)\right)\right) & \text { otherwise }\end{cases}
$$

The well-definedness of $F$ results from (3.4). It is skew symmetric by construction since $\succsim$ is complete. Let us show that $F$ is nondecreasing in its first $n$ arguments. Suppose that $u_{i}\left(z_{i}\right)>u_{i}\left(x_{i}\right)$ so that $z_{i} \succ_{i}^{ \pm} x_{i}$. If $x \succ y$, we know, using (3.3), that $\left(z_{i}, x_{-i}\right) \succ y$ and the conclusion follows from the definition of $F$. If $x \sim y$, we have, using $(3.3),\left(z_{i}, x_{-i}\right) \succsim y$ and the conclusion follows from the definition of $F$. If $\operatorname{Not}[x \succsim y]$ we have either $\left(z_{i}, x_{-i}\right) \succ y,\left(z_{i}, x_{-i}\right) \sim y$, or $\operatorname{Not}\left[\left(z_{i}, x_{-i}\right) \succsim y\right]$. In either case, the conclusion follows from the definition of $F$. The proof that $F$ is nonincreasing in its last $n$ argument is similar and is omitted. 
Part 6. In view of the proof of part 5 above, since $T A C 1$ and $T A C 2$ imply $A C 1$, $A C 2$ and $A C 3$ when $\succsim$ is complete, sufficiency follows from lemma 4.5.

\section{Corollary 4.8 (Links between models).}

(1) Models (L4) and (L3) are equivalent.

(2) Models (L6) and (L5) are equivalent.

(3) Model (L7) $\Rightarrow$ Model (L6).

PROOF. All parts directly results from lemma 4.6 and theorem 4.7.

\subsubsection{Remarks}

Remark 4.9. A somewhat weaker form (using nondecreasingness and nonincreasingness) of part 4 of theorem 4.7 was noted in Greco et al. (2002) using our conditions $A C 1, A C 2$ and $A C 3$.

Remark 4.10 (Skew symmetry and the rôle of 0). It should be observed that increasingness and nondecreasingness are equivalent in our models except in the case of a "skew symmetric" function $F$ (i.e., such that $F\left(\left[u_{i}\left(x_{i}\right)\right]\right.$; $\left.\left.\left[u_{i}\left(y_{i}\right)\right]\right)=-F\left(\left[u_{i}\left(y_{i}\right)\right] ;\left[u_{i}\left(x_{i}\right)\right]\right)\right)$. When $F$ is skew symmetric, the value " 0 " plays a special rôle. This leads to distinguish the increasing case from the nondecreasing one, as in proposition 2.6 with semiorders and weak orders. This will often be the case below.

Remark 4.11 (Uniqueness). It should be clear that the numerical representations envisaged in this section (see theorem 4.7) do not possess any remarkable uniqueness properties. It is not difficult however to analyze them. We take the case of model $(L 4)$.

Our proof shows that it is always possible to use functions $u_{i}$ such that:

$$
x_{i} \succsim_{i}^{ \pm} y_{i} \Leftrightarrow u_{i}\left(x_{i}\right) \geq u_{i}\left(y_{i}\right)
$$

This defines what could be called a regular representation of $(L 4)$. From the proof of theorem 4.7, it is clear that any $u_{i}$ satisfying (4.8) may be used, i.e. we may apply, independently on each attribute, any increasing transformation to the functions $u_{i}$ without altering the representation.

Other choices for $u_{i}$ are possible however. Let us show that any function $u_{i}$ such that:

$$
x_{i} \succ_{i}^{ \pm} y_{i} \Rightarrow u_{i}\left(x_{i}\right)>u_{i}\left(y_{i}\right),
$$

can be used in a representation of model $(L 4)$. 
The necessity of (4.9) is clear since $x_{i} \succ_{i}^{ \pm} y_{i}$ implies either $x_{i} \succ_{i}^{+} y_{i}$ or $x_{i} \succ_{i}^{-} y_{i}$. In the first case, we know that $\left(x_{i}, a_{-i}\right) \succsim z$ and $\operatorname{Not}\left[\left(y_{i}, a_{-i}\right) \succsim z\right]$, for some $z \in X$ and some $a_{-i} \in X_{-i}$. In the second case, we obtain $w \succsim\left(y_{i}, b_{-i}\right)$ and $\operatorname{Not}\left[w \succsim\left(x_{i}, b_{-i}\right)\right]$, for some $w \in X$ and some $b_{-i} \in X_{-i}$. Using the increasingness of $F$, either case implies $u_{i}\left(x_{i}\right)>u_{i}\left(y_{i}\right)$.

Conversely, it is clear that if $u_{i}$ satisfies (4.9) then

$$
u_{i}\left(x_{i}\right)=u_{i}\left(y_{i}\right) \Rightarrow x_{i} \sim_{i}^{ \pm} y_{i},
$$

so that defining $F$ using (4.6) as in the proof of theorem 4.7 leads to a welldefined function being increasing in its first $n$ arguments and decreasing in its last $n$ arguments.

It should be noted that any nonnegative (resp. negative) real-valued function $f$ (resp. $g$ ) on $\mathbb{R}^{2 n}$ that is increasing in its first $n$ arguments and decreasing in its last $n$ arguments when restricted to $\left[\prod_{i=1}^{n} u_{i}\left(X_{i}\right)\right]^{2}$ may be used to define $F$ letting $F\left(\left[u_{i}\left(x_{i}\right)\right] ;\left[u_{i}\left(y_{i}\right)\right]\right)=f\left(\left[u_{i}\left(x_{i}\right)\right] ;\left[u_{i}\left(y_{i}\right)\right]\right)$ if $x \succsim y$ and $F\left(\left[u_{i}\left(x_{i}\right)\right] ;\left[u_{i}\left(y_{i}\right)\right]\right)=g\left(\left[u_{i}\left(x_{i}\right)\right] ;\left[u_{i}\left(y_{i}\right)\right]\right)$ otherwise. It is not difficult to see that only such functions may be used.

We have therefore described the set of all possible numerical representations in model $(L 4)$. It is not difficult to adapt the above reasoning to cover all the models envisaged in this section (see Bouyssou and Pirlot, 2002f, for details).

Remark 4.12 (Weak orders). When $\succsim$ is a weak order, marginal traces on levels are confounded with marginal preferences. Hence, the above results can be greatly simplified. It is easy to see that, in this case,

- $[\succsim$ is weakly separable $] \Leftrightarrow[\succsim$ satisfies $A C 1] \Leftrightarrow[\succsim$ satisfies $A C 2] \Leftrightarrow[\succsim$ satisfies $A C 3]$,

- $[\succsim$ is weakly independent $] \Leftrightarrow[\succsim$ satisfies $T A C 12]$.

Using these observations, it is easy to show that, when $\succsim$ is a weak order on a countable set $X=\prod_{i=1}^{n} X_{i}$, it always has a representation such that:

$$
x \succsim y \Leftrightarrow U\left(u_{1}\left(x_{1}\right), \ldots, u_{n}\left(x_{n}\right)\right) \geq U\left(u_{1}\left(y_{1}\right), \ldots, u_{n}\left(y_{n}\right)\right) .
$$

Furthermore, $U$ can be chosen so that it is nondecreasing in all its arguments (resp. increasing) if and only if $\succsim$ is weakly separable (resp. weakly independent).

Whereas the increasing case is well-known Krantz et al. (1971, theorem 7.1), the result in the nondecreasing case generalizes a result obtained in Blackorby et al. (1978) in the case in which $X \subseteq \mathbb{R}^{n}$.

Remark 4.13 (Extension to the general case). Most of our results are 
technically simple. Their extension to the general case (i.e. removing the hypothesis that $X$ is countable) do not raise any serious difficulty beyond the well-known one of guaranteeing that equivalences and/or weak orders have a numerical representation (see Bouyssou and Pirlot, 2002f).

Remark 4.14 (Left and right traces on levels). For the sake of conciseness, we do not investigate here all possible models involving marginal traces on levels. Let us simply mention that the case in which $A C 1$ and $A C 2$ hold but not $A C 3$ is of particular interest. The similarity of this case with that of interval orders (see Fishburn, 1970a, 1973, 1985) should be clear at this point. Most of our results can easily be modified to cover this case using a representation of the type:

$$
x \succsim y \Leftrightarrow F\left(\left[v_{i}\left(x_{i}\right)\right] ;\left[w_{i}\left(y_{i}\right)\right]\right) \geq 0,
$$

leaving room for relations in which the marginal traces $\succsim_{i}^{+}$and $\succsim_{i}^{-}$may not be compatible.

Remark 4.15 (Other extensions). We restricted our attention in this paper to the analysis of conditions $A C 1_{i}, A C 2_{i}, A C 3_{i}, T A C 1_{i}$ and $T A C 2_{i}$ when imposed on all $i \in N$. As observed in Greco et al. (2002), this might be overly restrictive. It is not difficult however to study the, rather awkward, models that obtain when these conditions are only imposed on some, but not all attributes. They amount to supposing that $F$ is increasing (resp. decreasing) in some, but not necessarily all, of its $n$ first (resp. last arguments).

Similarly, it is easy to generalize our conditions to subsets of attributes more general than a singleton. The study of the resulting models certainly deserves attention. In fact, when aggregating attributes, it might well happen that attributes interact in such a way that weak separability is violated (remember the famous example of the choice a dinner involving two attributes: the main course and the wine). This would forbid the use of $A C 1$ or $A C 2$ as done here. Imposing these conditions on the groups of "strongly interacting" attributes might however lead to useful models. Such models would be in the spirit of the process of "building criteria" by sub-aggregation as described in Bouyssou (1990); Roy (1996).

The main results in this section are summarized in table 4 and figure 2 .

\section{Models using marginal traces on differences ${ }^{4}$}

$\overline{4}$ This section is based on Bouyssou and Pirlot (2002d) 
Table 4

Main results using traces on levels

\begin{tabular}{|c|c|c|}
\hline Models & Definition & Conditions \\
\hline$(L 0)$ & $x \succsim y \Leftrightarrow F\left(\left[u_{i}\left(x_{i}\right)\right],\left[u_{i}\left(y_{i}\right)\right]\right) \geq 0$ & $\varnothing$ \\
\hline$(L 1)$ & $(L 0)$ with $F\left(\left[u_{i}\left(x_{i}\right)\right],\left[u_{i}\left(x_{i}\right)\right]\right)=0$ & refl. \\
\hline$(L 2)$ & $\begin{array}{c}(L 1) \text { with } \\
F\left(\left[u_{i}\left(x_{i}\right)\right] ;\left[u_{i}\left(y_{i}\right)\right]\right)=-F\left(\left[u_{i}\left(y_{i}\right)\right] ;\left[u_{i}\left(x_{i}\right)\right]\right)\end{array}$ & cpl. \\
\hline$(L 3) \Leftrightarrow(L 4)$ & $(L 0)$ with $F(\nearrow \nearrow, \searrow \searrow)$ & $A C 123$ \\
\hline$(L 5) \Leftrightarrow(L 6)$ & $(L 1)$ with $F(\nearrow \nearrow, \searrow \searrow)$ & refl., $A C 123$ \\
\hline$(L 7)$ & $(L 2)$ with $F(\nearrow, \searrow)$ & cpl., $A C 123$ \\
\hline$(L 8)$ & $(L 2)$ with $F(\nearrow \nearrow, \searrow)$ & cpl., TAC12 \\
\hline \multicolumn{3}{|c|}{ 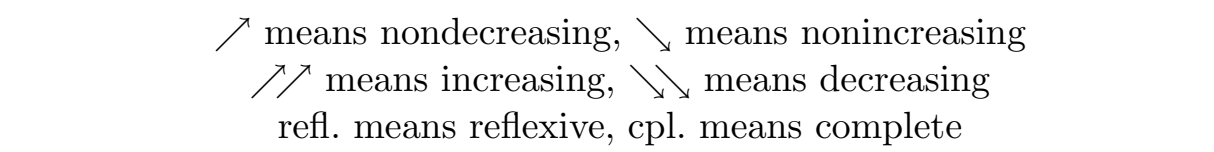 } \\
\hline
\end{tabular}

Figure 2. Implication between models resulting from theorem 4.7

$$
\begin{array}{ccc}
(L 8) & & \\
\Downarrow \\
(L 7)
\end{array} \quad \Rightarrow \quad(L 2)
$$

All models imply $(L 0)$

This section follows the same path as in section 4 replacing marginal traces on levels by marginal traces on differences.

\subsection{Complete marginal traces on differences}

Definition 5.1 (Conditions $R C 1$ and $R C 2$ ). Let $\succsim$ be a binary relation on a set $X=\prod_{i=1}^{n} X_{i}$. For $i \in N$, this relation is said to satisfy:

$R C 1_{i}$ if

$$
\left.\begin{array}{c}
\left(x_{i}, a_{-i}\right) \succsim\left(y_{i}, b_{-i}\right) \\
\text { and } \\
\left(z_{i}, c_{-i}\right) \succsim\left(w_{i}, d_{-i}\right)
\end{array}\right\} \Rightarrow\left\{\begin{array}{l}
\left(x_{i}, c_{-i}\right) \succsim\left(y_{i}, d_{-i}\right) \\
\text { or } \\
\left(z_{i}, a_{-i}\right) \succsim\left(w_{i}, b_{-i}\right),
\end{array}\right.
$$


$R C 2_{i}$ if

$$
\left.\begin{array}{c}
\left(x_{i}, a_{-i}\right) \succsim\left(y_{i}, b_{-i}\right) \\
\text { and } \\
\left(y_{i}, c_{-i}\right) \succsim\left(x_{i}, d_{-i}\right)
\end{array}\right\} \Rightarrow\left\{\begin{array}{l}
\left(z_{i}, a_{-i}\right) \succsim\left(w_{i}, b_{-i}\right) \\
\text { or } \\
\left(w_{i}, c_{-i}\right) \succsim\left(z_{i}, d_{-i}\right),
\end{array}\right.
$$

for all $x_{i}, y_{i}, z_{i}, w_{i} \in X_{i}$ and all $a_{-i}, b_{-i}, c_{-i}, d_{-i} \in X_{-i}$. We say that $\succsim$ satisfies $R C 1$ (resp. $R C 2$ ) if it satisfies $R C 1_{i}$ (resp. $R C 2_{i}$ ) for all $i \in N$. We sometimes write $R C 12$ as shorthand for $R C 1$ and $R C 2$.

Condition $R C 1_{i}$ suggests that either $\left(x_{i}, y_{i}\right)$ is a larger preference difference than $\left(z_{i}, w_{i}\right)$ or vice versa. Indeed, it is easy to see that supposing $\operatorname{Not}\left[\left(x_{i}, y_{i}\right) \succsim_{i}^{*}\right.$ $\left.\left(z_{i}, w_{i}\right)\right]$ and $\operatorname{Not}\left[\left(z_{i}, w_{i}\right) \succsim_{i}^{*}\left(x_{i}, y_{i}\right)\right]$ leads to a violation of $R C 1_{i}$. Hence, $R C 1_{i}$ is equivalent to supposing the completeness of $\succsim_{i}^{*}$. Similarly, $R C 2_{i}$ suggests that the two opposite differences $\left(x_{i}, y_{i}\right)$ and $\left(y_{i}, x_{i}\right)$ are linked. In terms of the relation $\succsim_{i}^{*}$, it says that if the preference difference between $x_{i}$ and $y_{i}$ is not at least as large as the preference difference between $z_{i}$ and $w_{i}$ then the preference difference between $y_{i}$ and $x_{i}$ should be at least as large as the preference difference between $w_{i}$ and $z_{i}$. We summarize these observations in the following lemma; we omit its straightforward proof.

Lemma 5.2 (Complete Traces on differences). We have:

(1) $\left[\succsim_{i}^{*}\right.$ is complete $] \Leftrightarrow R C 1_{i}$,

(2) $R C 2_{i} \Leftrightarrow$ [for all $\left.x_{i}, y_{i}, z_{i}, w_{i} \in X_{i}, N o t\left[\left(x_{i}, y_{i}\right) \succsim_{i}^{*}\left(z_{i}, w_{i}\right)\right] \Rightarrow\left(y_{i}, x_{i}\right) \succsim_{i}^{*}\left(w_{i}, z_{i}\right)\right]$,

(3) $\left[\succsim_{i}^{* *}\right.$ is complete $] \Leftrightarrow\left[R C 1_{i}\right.$ and $\left.R C 2_{i}\right]$.

Condition $R C 1$ was introduced in Bouyssou (1986) under the name "weak cancellation". Technically $R C 1_{i}$ amounts to defining a biorder, in the sense of Ducamp and Falmagne (1969) and Doignon, Ducamp, and Falmagne (1984), between the sets $X_{i}^{2}$ and $X_{-i}^{2}$. The extension of condition $R C 1$ to subsets of attributes is central in Vind (1991) where this condition is called "independence". Condition $R C 2$ was first proposed in Bouyssou et al. (1997). We note below a number of other important facts about $R C 1$ and $R C 2$.

\section{Lemma 5.3 (Consequences of $R C 1$ and $R C 2$ ).}

(1) If $\succsim$ satisfies $R C 1_{i}$ then it is weakly separable for $i \in N$,

(2) If $\succsim$ satisfies $R C 2$ then it is independent and either reflexive or irreflexive,

(3) Reflexivity, independence and $R C 1$ are independent conditions,

(4) In the class of complete relations, $R C 1$ and $R C 2$ are independent conditions.

PROOF. Part 1. Suppose that $\left(x_{i}, a_{-i}\right) \succ\left(x_{i}, b_{-i}\right)$ and $\left(y_{i}, b_{-i}\right) \succ\left(y_{i}, a_{-i}\right)$. This implies $\left(x_{i}, a_{-i}\right) \succsim\left(x_{i}, b_{-i}\right)$ and $\left(y_{i}, b_{-i}\right) \succsim\left(y_{i}, a_{-i}\right)$ so that $R C 1_{i}$ implies either $\left(y_{i}, a_{-i}\right) \succsim\left(y_{i}, b_{-i}\right)$ or $\left(x_{i}, b_{-i}\right) \succsim\left(x_{i}, a_{-i}\right)$, a contradiction. 
Part 2. If $\left(x_{i}, a_{-i}\right) \succsim\left(x_{i}, b_{-i}\right), R C 2_{i}$ implies $\left(y_{i}, a_{-i}\right) \succsim\left(y_{i}, b_{-i}\right)$ for all $y_{i} \in X_{i}$ so that $\succsim$ is independent. It is clear that an independent relation is either reflexive or irreflexive.

Part 3. In order to show that these three properties are completely independent, we need $2^{3}=8$ examples. It is easy to build a relation $\succsim$ that does not satisfy $R C 1$ and is neither reflexive nor independent (e.g. take $X=$ $\{a, b\} \times\{z, w\}$ and let $\succsim$ be an empty relation on $X$ except that $(a, z) \succsim(b, z)$ and $(b, w) \succsim(a, w))$. Any relation $\succsim$ satisfying the additive utility model satisfies the three properties. The other six examples are provided in appendix A.2.

Part 4 . Any relation $\succsim$ satisfying the additive utility model is complete and satisfies both $R C 1$ and $R C 2$. We provide in appendix A.2 the three remaining examples.

\subsection{Strict responsiveness to traces on differences}

Definition 5.4 (Condition $T C$ ). Let $\succsim$ be a binary relation on a set $X=$ $\prod_{i=1}^{n} X_{i}$. For $i \in N$, this relation is said to satisfy:

$T C_{i}$ if

$$
\left.\begin{array}{l}
\left(x_{i}, a_{-i}\right) \succsim\left(y_{i}, b_{-i}\right) \\
\text { and } \\
\left(z_{i}, b_{-i}\right) \succsim\left(w_{i}, a_{-i}\right) \\
\text { and } \\
\left(w_{i}, c_{-i}\right) \succsim\left(z_{i}, d_{-i}\right)
\end{array}\right\} \Rightarrow\left(x_{i}, c_{-i}\right) \succsim\left(y_{i}, d_{-i}\right),
$$

for all $x_{i}, y_{i}, z_{i}, w_{i} \in X_{i}$ and all $a_{-i}, b_{-i}, c_{-i}, d_{-i} \in X_{-i}$. We say that $\succsim$ satisfies $T C$ if it satisfies $T C_{i}$ for all $i \in N$.

Condition $T C_{i}$ (Triple Cancellation) is a classical cancellation condition that has been often used (see Krantz et al., 1971; Wakker, 1989) in the analysis of the additive utility model (1.1). As shown below, it implies both $R C 1$ and $R C 2$ when $\succsim$ is complete. We refer to Wakker $(1988,1989)$ for a detailed analysis of $T C$ including its interpretation in terms of difference of preference.

As soon as $\succsim$ is complete, the following lemma shows that $T C_{i}$ is exactly what is needed to ensure the strict responsiveness of $\succsim$ w.r.t. $\succsim_{i}^{* *}$.

\section{Lemma 5.5 (Strict responsiveness to marginal traces on differences).}

(1) If $\succsim$ is complete, $T C_{i}$ implies $R C 1_{i}$ and $R C 2_{i}$, 
(2) If $T C_{i}$ holds and $\succsim ~ i s ~ c o m p l e t e, ~\left[x \succsim y\right.$ and $\left.\left(z_{i}, w_{i}\right) \succ_{i}^{* *}\left(x_{i}, y_{i}\right)\right] \Rightarrow$ $\left(z_{i}, x_{-i}\right) \succ\left(w_{i}, y_{-i}\right)$.

PROOF. Part 1. In contradiction with $R C 1_{i}$, suppose that $\left(x_{i}, a_{-i}\right) \succsim\left(y_{i}, b_{-i}\right)$, $\left(z_{i}, c_{-i}\right) \succsim\left(w_{i}, d_{-i}\right), \operatorname{Not}\left[\left(z_{i}, a_{-i}\right) \succsim\left(w_{i}, b_{-i}\right)\right]$ and $\operatorname{Not}\left[\left(x_{i}, c_{-i}\right) \succsim\left(y_{i}, d_{-i}\right)\right]$. Since $\succsim$ is complete, we have $\left(w_{i}, b_{-i}\right) \succ\left(z_{i}, a_{-i}\right)$. Using $T C_{i},\left(x_{i}, a_{-i}\right) \succsim$ $\left(y_{i}, b_{-i}\right),\left(w_{i}, b_{-i}\right) \succ\left(z_{i}, a_{-i}\right)$ and $\left(z_{i}, c_{-i}\right) \succsim\left(w_{i}, d_{-i}\right)$ imply $\left(x_{i}, c_{-i}\right) \succsim\left(y_{i}, d_{-i}\right)$, a contradiction.

Similarly suppose, in contradiction with $R C 2_{i}$ that $\left(x_{i}, a_{-i}\right) \succsim\left(y_{i}, b_{-i}\right),\left(y_{i}, c_{-i}\right)$ $\succsim\left(x_{i}, d_{-i}\right), \operatorname{Not}\left[\left(z_{i}, a_{-i}\right) \succsim\left(w_{i}, b_{-i}\right)\right]$ and $\operatorname{Not}\left[\left(w_{i}, c_{-i}\right) \succsim\left(z_{i}, d_{-i}\right)\right]$. Since $\succsim$ is complete, we know that $\left(w_{i}, b_{-i}\right) \succ\left(z_{i}, a_{-i}\right)$. Using $T C_{i},\left(w_{i}, b_{-i}\right) \succ\left(z_{i}, a_{-i}\right)$, $\left(x_{i}, a_{-i}\right) \succsim\left(y_{i}, b_{-i}\right)$ and $\left(y_{i}, c_{-i}\right) \succsim\left(x_{i}, d_{-i}\right)$ imply $\left(w_{i}, c_{-i}\right) \succsim\left(z_{i}, d_{-i}\right)$, a contradiction.

Part 2. Using (3.6), we get $\left(z_{i}, x_{-i}\right) \succsim\left(w_{i}, y_{-i}\right)$. Suppose that $\left(w_{i}, y_{-i}\right) \succsim\left(z_{i}\right.$, $\left.x_{-i}\right)$. From part 1 and lemma 5.2, we know that $\succsim_{i}^{* *}$ is complete. We thus have $\left(z_{i}, w_{i}\right) \succ_{i}^{* *}\left(x_{i}, y_{i}\right) \Leftrightarrow \operatorname{Not}\left[\left(x_{i}, y_{i}\right) \succsim_{i}^{* *}\left(z_{i}, w_{i}\right)\right] \Leftrightarrow\left[\operatorname{Not}\left[\left(x_{i}, y_{i}\right) \succsim_{i}^{*}\left(z_{i}, w_{i}\right)\right]\right.$ or $\left.\operatorname{Not}\left[\left(w_{i}, z_{i}\right) \succsim_{i}^{*}\left(y_{i}, x_{i}\right)\right]\right]$. In the first case we have $\operatorname{Not}\left[\left(x_{i}, c_{-i}\right) \succsim\left(y_{i}\right.\right.$, $\left.\left.d_{-i}\right)\right]$ and $\left(z_{i}, c_{-i}\right) \succsim\left(w_{i}, d_{-i}\right)$, for some $c_{-i}, d_{-i} \in X_{-i}$. Using $T C_{i}, x \succsim y$, $\left(w_{i}, y_{-i}\right) \succsim\left(z_{i}, x_{-i}\right)$ and $\left(z_{i}, c_{-i}\right) \succsim\left(w_{i}, d_{-i}\right)$ imply $\left(x_{i}, c_{-i}\right) \succsim\left(y_{i}, d_{-i}\right)$, a contradiction. The other case is similar.

\subsection{Marginal traces on differences and numerical representations}

Starting from the trivial model $(D 0)$, introduced in section 3.5 , in which:

$$
x \succsim y \Leftrightarrow G\left(\left[p_{i}\left(x_{i}, y_{i}\right)\right]\right) \geq 0
$$

we envisage:

- model $(D 1)$ adding to $(D 0)$ the fact that $p_{i}\left(x_{i}, x_{i}\right)=0$, for all $i \in N$ and all $x_{i} \in X_{i}$,

- model $(D 2)$ adding to $(D 1)$ the skew symmetry of each $p_{i}$, i.e. $p_{i}\left(x_{i}, y_{i}\right)=$ $-p_{i}\left(y_{i}, x_{i}\right)$, for all $i \in N$ and all $x_{i}, y_{i} \in X_{i}$,

- model (D3) adding to (D2) the oddness of $G$, i.e. $G(\mathbf{x})=-G(-\mathbf{x})$, abusing notation in an obvious way.

As before, we furthermore envisage the consequences of supposing that in each of the above four models ((D0), (D1), (D2) and $(D 3)), G$ is nondecreasing or increasing in each of its arguments. This leads to a total of 12 different models, the definition of which being summarized in table 5 . 
Table 5

Models involving traces on differences

\begin{tabular}{|c|c|}
\hline $\begin{array}{l}(D 0) \\
(D 1) \\
(D 2) \\
(D 3)\end{array}$ & $\begin{array}{c}x \succsim y \Leftrightarrow G\left(\left[p_{i}\left(x_{i}, y_{i}\right)\right]\right) \geq 0 \\
(D 0) \text { with } p_{i}\left(x_{i}, x_{i}\right)=0 \\
(D 1) \text { with } p_{i}\left(x_{i}, y_{i}\right)=-p_{i}\left(y_{i}, x_{i}\right) \\
(D 2) \text { with } G \text { odd }\end{array}$ \\
\hline $\begin{array}{l}(D 4) \\
(D 8)\end{array}$ & $\begin{array}{l}(D 0) \text { with } G \text { nondecreasing } \\
(D 0) \text { with } G \text { increasing }\end{array}$ \\
\hline $\begin{array}{l}(D 5) \\
(D 9)\end{array}$ & $\begin{array}{l}(D 1) \text { with } G \text { nondecreasing } \\
(D 1) \text { with } G \text { increasing }\end{array}$ \\
\hline $\begin{array}{l}(D 6) \\
(D 10)\end{array}$ & $\begin{array}{l}(D 2) \text { with } G \text { nondecreasing } \\
(D 2) \text { with } G \text { increasing }\end{array}$ \\
\hline $\begin{array}{l}(D 7) \\
(D 11)\end{array}$ & $\begin{array}{c}\text { (D3) with } G \text { nondecreasing } \\
(D 3) \text { with } G \text { increasing }\end{array}$ \\
\hline
\end{tabular}

The implications between these various models are clear from their definitions. They are summarized in figure 3. The following lemma takes note of the rela-

Figure 3. Implication between models involving traces on differences

\begin{tabular}{|c|c|c|c|c|c|c|c|}
\hline$(D 11)$ & $\Rightarrow$ & $(D 10)$ & $\Rightarrow$ & $(D 9)$ & $\Rightarrow$ & $(D 8)$ & inc. \\
\hline$\Downarrow$ & & $\Downarrow$ & & $\Downarrow$ & & $\Downarrow$ & \\
\hline$(D 7)$ & $\Rightarrow$ & $(D 6)$ & $\Rightarrow$ & $(D 5)$ & $\Rightarrow$ & $(D 4)$ & nondec \\
\hline $\begin{array}{c}\Downarrow \\
(D 3)\end{array}$ & $\Rightarrow$ & $\begin{array}{c}\Downarrow \\
(D 2)\end{array}$ & $\Rightarrow$ & $\begin{array}{c}\Downarrow \\
(D 1)\end{array}$ & $\Rightarrow$ & $\begin{array}{c}\Downarrow \\
(D 0)\end{array}$ & $\varnothing$ \\
\hline$G$ odd & & $p_{i}$ sk-sym. & & $p_{i}\left(x_{i}, x_{i}\right)=0$ & & $\varnothing$ & \\
\hline
\end{tabular}

inc. means increasing in all arguments nondec. means nondecreasing in all arguments sk-sym. means skew symmetric

tions between these models and our conditions involving traces on differences.

\section{Lemma 5.6 (Necessary conditions).}

(1) Model (D1) implies that $\succsim$ is independent,

(2) Model (D3) implies that $\succsim$ is complete,

(3) Model (D4) implies RC1,

(4) Model (D6) implies RC2,

(5) Model (D11) implies TC, 
PROOF. Part 1. In model $(D 1)$, we have $p_{i}\left(x_{i}, x_{i}\right)=0$, so that $\left(x_{i}, a_{-i}\right) \succsim$ $\left(x_{i}, b_{-i}\right) \Leftrightarrow G\left(0,\left(p_{j}\left(a_{j}, b_{j}\right)\right)_{j \neq i}\right) \geq 0 \Leftrightarrow\left(y_{i}, a_{-i}\right) \succsim\left(y_{i}, b_{-i}\right)$. Hence $\succsim$ is independent.

Part 2. By definition of model $(D 3), N o t[x \succsim y] \Leftrightarrow G\left(\left[p_{i}\left(x_{i}, y_{i}\right)\right]\right)<0$ so that, using the skew symmetry of the $p_{i}$ 's and the oddness of $G, G\left(\left[p_{i}\left(y_{i}, x_{i}\right)\right]\right)>0$ which implies $y \succsim x$. Hence $\succsim$ is complete.

Part 3. Suppose that $\left(x_{i}, a_{-i}\right) \succsim\left(y_{i}, b_{-i}\right)$ and $\left(z_{i}, c_{-i}\right) \succsim\left(w_{i}, d_{-i}\right)$. Using model (D4) we have:

$$
\begin{aligned}
& G\left(p_{i}\left(x_{i}, y_{i}\right),\left(p_{j}\left(a_{j}, b_{j}\right)\right)_{j \neq i}\right) \geq 0 \text { and } \\
& G\left(p_{i}\left(z_{i}, w_{i}\right),\left(p_{j}\left(c_{j}, d_{j}\right)\right)_{j \neq i}\right) \geq 0 .
\end{aligned}
$$

If $p_{i}\left(x_{i}, y_{i}\right) \geq p_{i}\left(z_{i}, w_{i}\right)$, then using the nondecreasingness of $G$, we have $G\left(p_{i}\left(x_{i}, y_{i}\right),\left(p_{j}\left(c_{j}, d_{j}\right)\right)_{j \neq i}\right) \geq 0$ so that $\left(x_{i}, c_{-i}\right) \succsim\left(y_{i}, d_{-i}\right)$. If $p_{i}\left(z_{i}, w_{i}\right)>$ $p_{i}\left(x_{i}, y_{i}\right)$, we have $G\left(p_{i}\left(z_{i}, w_{i}\right),\left(p_{j}\left(a_{j}, b_{j}\right)\right)_{j \neq i}\right) \geq 0$ so that $\left(z_{i}, a_{-i}\right) \succsim\left(w_{i}, b_{-i}\right)$. Therefore $R C 1$ holds.

Part 4. Suppose that $\left(x_{i}, a_{-i}\right) \succsim\left(y_{i}, b_{-i}\right)$ and $\left(y_{i}, c_{-i}\right) \succsim\left(x_{i}, d_{-i}\right)$. Using model (D6) we have:

$$
\begin{aligned}
& G\left(p_{i}\left(x_{i}, y_{i}\right),\left(p_{j}\left(a_{j}, b_{j}\right)\right)_{j \neq i}\right) \geq 0 \text { and } \\
& G\left(p_{i}\left(y_{i}, x_{i}\right),\left(p_{j}\left(c_{j}, d_{j}\right)\right)_{j \neq i}\right) \geq 0 .
\end{aligned}
$$

If $p_{i}\left(x_{i}, y_{i}\right) \geq p_{i}\left(z_{i}, w_{i}\right)$, the skew symmetry of $p_{i}$ implies $p_{i}\left(w_{i}, z_{i}\right) \geq p_{i}\left(y_{i}, x_{i}\right)$ so that $\left(w_{i}, c_{-i}\right) \succsim\left(z_{i}, d_{-i}\right)$ using the nondecreasingness of $G$. Similarly, if $p_{i}\left(z_{i}, w_{i}\right)>p_{i}\left(x_{i}, y_{i}\right)$ we have, using the nondecreasingness of $G,\left(z_{i}, a_{-i}\right) \succsim$ $\left(w_{i}, b_{-i}\right)$. Therefore, $R C 2$ holds.

Part 5. Suppose that $\left(x_{i}, a_{-i}\right) \succsim\left(y_{i}, b_{-i}\right),\left(z_{i}, b_{-i}\right) \succsim\left(w_{i}, a_{-i}\right),\left(w_{i}, c_{-i}\right) \succsim\left(z_{i}\right.$, $\left.d_{-i}\right)$ and $\operatorname{Not}\left[\left(x_{i}, c_{-i}\right) \succsim\left(y_{i}, d_{-i}\right)\right]$. Using model $(D 11)$ we know that:

$$
\begin{aligned}
& G\left(p_{i}\left(x_{i}, y_{i}\right),\left(p_{j}\left(a_{j}, b_{j}\right)\right)_{j \neq i}\right) \geq 0, \\
& G\left(p_{i}\left(z_{i}, w_{i}\right),\left(p_{j}\left(b_{j}, a_{j}\right)\right)_{j \neq i}\right) \geq 0, \\
& G\left(p_{i}\left(w_{i}, z_{i}\right),\left(p_{j}\left(c_{j}, d_{j}\right)\right)_{j \neq i}\right) \geq 0 \text { and } \\
& G\left(p_{i}\left(x_{i}, y_{i}\right),\left(p_{j}\left(c_{j}, d_{j}\right)\right)_{j \neq i}\right)<0 .
\end{aligned}
$$

Using the oddness of $G$, its increasingness and the skew symmetry of the $p_{i}$ 's, the first two inequalities imply $p_{i}\left(x_{i}, y_{i}\right) \geq p_{i}\left(w_{i}, z_{i}\right)$ whereas the last two imply that $p_{i}\left(x_{i}, y_{i}\right)<p_{i}\left(w_{i}, z_{i}\right)$, a contradiction.

We are now in position to characterize our models involving traces on differences. 
Theorem 5.7 (Models using traces on differences). Let $\succsim$ be a binary relation on a countable set $X=\prod_{i=1}^{n} X_{i}$.

(1) Model (D2) holds iff $\succsim$ is independent,

(2) Model (D3) holds iff $\succsim$ is complete and independent,

(3) Model (D8) holds iff $\succsim$ satisfies RC1,

(4) Model (D9) holds iff $\succsim$ is independent and satisfies $R C 1$,

(5) Model (D10) holds iff $\succsim$ satisfies $R C 1$ and $R C 2$,

(6) Model (D7) holds iff $\succsim$ is complete and satisfies $R C 1$ and $R C 2$,

(7) Model (D11) holds iff $\succsim$ is complete and satisfies TC.

PROOF. Necessity of all parts results from lemma 5.6 and the implications between our models. We show sufficiency.

Part 1. Since $\succsim$ is independent, we know that, for all $i \in N$ and all $x_{i}, y_{i} \in X_{i}$, $\left(x_{i}, x_{i}\right) \sim_{i}^{*}\left(y_{i}, y_{i}\right)$ and, thus, $\left(x_{i}, x_{i}\right) \sim_{i}^{* *}\left(y_{i}, y_{i}\right)$. Since, $X_{i}$ is countable, there is a one-to-one correspondence $p_{i}$ between $X_{i}^{2} \backslash\left\{\left(x_{i}, x_{i}\right): x_{i} \in X_{i}\right\}$ and some countable subset of $\mathbb{R}$. It can always be chosen so that, for all $x_{i}, y_{i} \in X_{i}$, $p_{i}\left(x_{i}, y_{i}\right)=-p_{i}\left(y_{i}, x_{i}\right)$. Let $p_{i}\left(x_{i}, x_{i}\right)=0$, for all $x_{i} \in X_{i}$. By construction, $p_{i}$ is skew symmetric. Furthermore, we have:

$$
p_{i}\left(x_{i}, y_{i}\right)=p_{i}\left(z_{i}, w_{i}\right) \Leftrightarrow\left\{\begin{array}{c}
x_{i}=y_{i} \text { and } z_{i}=w_{i} \\
\text { or } \\
x_{i}=z_{i} \text { and } y_{i}=w_{i} .
\end{array}\right.
$$

Consider, on all $i \in N$, a function $p_{i}$ satisfying (5.1) and define $G$ on $\prod_{i=1}^{n} p_{i}\left(X_{i}^{2}\right)$ letting:

$$
G\left(\left[p_{i}\left(x_{i}, y_{i}\right)\right]\right)= \begin{cases}+1 & \text { if } x \succsim y \\ -1 & \text { otherwise. }\end{cases}
$$

Since, by hypothesis, $\left(x_{i}, x_{i}\right) \sim_{i}^{*}\left(y_{i}, y_{i}\right)$, it is easy to see that that $G$ is welldefined.

Part 2. Consider functions $p_{i}$ defined as in part 1 and define $G$ on $\prod_{i=1}^{n} p_{i}\left(X_{i}^{2}\right)$ letting:

$$
G\left(\left[p_{i}\left(x_{i}, y_{i}\right)\right]\right)=\left\{\begin{aligned}
+1 & \text { if } x \succ y \\
0 & \text { if } x \sim y \\
-1 & \text { otherwise }
\end{aligned}\right.
$$

Since, by hypothesis, $\left(x_{i}, x_{i}\right) \sim_{i}^{*}\left(y_{i}, y_{i}\right)$, it is easy to see that $G$ is well-defined. It is odd since $\succsim$ is complete.

Part 3. Since $R C 1$ holds, we know that $\succsim_{i}^{*}$ is a weak order. Since $X_{i}$ is countable, there is a real-valued function $p_{i}$ on $X_{i}^{2}$ such that:

$$
\left(x_{i}, y_{i}\right) \succsim_{i}^{*}\left(z_{i}, w_{i}\right) \Leftrightarrow p_{i}\left(x_{i}, y_{i}\right) \geq p_{i}\left(z_{i}, w_{i}\right) .
$$


Given such a particular numerical representation $p_{i}$ of $\succsim_{i}^{*}$ for $i=1,2, \ldots, n$, define $G$ on $p_{i}\left(X_{i}^{2}\right)$ as follows:

$$
G\left(\left[p_{i}\left(x_{i}, y_{i}\right)\right]\right)= \begin{cases}+\exp \left(\sum_{i=1}^{n} p_{i}\left(x_{i}, y_{i}\right)\right) & \text { if } x \succsim y \\ -\exp \left(-\sum_{i=1}^{n} p_{i}\left(x_{i}, y_{i}\right)\right) & \text { otherwise. }\end{cases}
$$

The well-definedness of $G$ follows from (3.7) and the definition of the $p_{i}$ 's. To show that $G$ is increasing, suppose that $p_{i}\left(z_{i}, w_{i}\right)>p_{i}\left(x_{i}, y_{i}\right)$, i.e. that $\left(z_{i}, w_{i}\right) \succ_{i}^{*}\left(x_{i}, y_{i}\right)$. If $x \succsim y$, we know from (3.6) that $\left(z_{i}, x_{-i}\right) \succsim\left(w_{i}, y_{-i}\right)$ and the conclusion follows from the definition of $G$. If $\operatorname{Not}[x \succsim y]$ we have either $\operatorname{Not}\left[\left(z_{i}, x_{-i}\right) \succsim\left(w_{i}, y_{-i}\right)\right]$ or $\left(z_{i}, x_{-i}\right) \succsim\left(w_{i}, y_{-i}\right)$. In either case, the conclusion follows from the definition of $G$.

Part 4 immediately follows from part 3 . Indeed, when $\succsim$ is independent, $\left(x_{i}, x_{i}\right) \sim_{i}^{*}\left(y_{i}, y_{i}\right)$, for all $i \in N$ and all $x_{i}, y_{i} \in X_{i}$. It is therefore always possible to choose a function $p_{i}$ satisfying (5.4) so that $p_{i}\left(x_{i}, x_{i}\right)=0$.

Part 5. Since $R C 1_{i}$ and $R C 2_{i}$ hold, we know from lemma 5.3 that $\succsim_{i}^{* *}$ is complete so that it is a weak order. This implies that $\succsim_{i}^{*}$ is a weak order and, since $X_{i}$ is countable, there is a real-valued function $q_{i}$ on $X_{i}^{2}$ satisfying (5.4). Given a particular numerical representation $q_{i}$ of $\succsim_{i}^{*}$, let $p_{i}\left(x_{i}, y_{i}\right)=$ $q_{i}\left(x_{i}, y_{i}\right)-q_{i}\left(y_{i}, x_{i}\right)$. It is obvious that $p_{i}$ is skew symmetric and represents $\succsim_{i}^{* *}$. Define $G$ as in (5.5). Its well-definedness results from (3.9). To show that $G$ is increasing, suppose that $p_{i}\left(z_{i}, w_{i}\right)>p_{i}\left(x_{i}, y_{i}\right)$, i.e. that $\left(z_{i}, w_{i}\right) \succ_{i}^{* *}\left(x_{i}, y_{i}\right)$. By construction, this implies $\left(z_{i}, w_{i}\right) \succsim_{i}^{*}\left(x_{i}, y_{i}\right)$. The increasingness of $G$ is therefore proved as in part 3 using (3.6).

Part 6. Define the functions $p_{i}$ as in part 5 and define $G$ letting

$$
G\left(\left[p_{i}\left(x_{i}, y_{i}\right)\right]\right)= \begin{cases}+\exp \left(\sum_{i=1}^{n} p_{i}\left(x_{i}, y_{i}\right)\right) & \text { if } x \succ y \\ 0 & \text { if } x \sim y \\ -\exp \left(-\sum_{i=1}^{n} p_{i}\left(x_{i}, y_{i}\right)\right) & \text { otherwise }\end{cases}
$$

Since $\succsim$ is complete, $G$ is odd. It is well-defined in view of (3.9) and the definition of the $p_{i}$ 's. Its nondecreasingness follows from (3.6) and (3.8).

Part 7. Since $T C$ holds and $\succsim$ is complete, we know from part 1 of lemma 5.5 that $R C 1$ and $R C 2$ hold. Define $p_{i}$ and $G$ as in part 6 . The increasingness of $F$ follows from part 2 of lemma 5.5.

\section{Corollary 5.8 (Links between models).}

(1) Models (D1) and (D2) are equivalent.

(2) Models (D4) and (D8) are equivalent.

(3) Models (D5) and (D9) are equivalent. 
(4) Models (D6) and (D10) are equivalent.

(5) Model (D7) $\Rightarrow$ Model (D10).

PROOF. All parts result directly from lemma 5.6 and theorem 5.7.

\subsection{Remarks}

Remark 5.9 (Goldstein (1991)). Models (D8) and (D4) were introduced by Goldstein (1991) as particular cases of his "decomposable thresholds models". He already noted their equivalence.

Remark 5.10 (Equivalence between models). Some care must be exercised when adding additional conditions to equivalent models. These additions may turn equivalent models into distinct ones. An example of this situation occurred in theorem 5.7. While models $(D 1)$ and $(D 2)$ are equivalent, this is no more the case when it is required that $G$ is nondecreasing in all its arguments. Models (D5) and (D6) are indeed distinct.

Remark 5.11 (Marginal preferences). The nontransitivity and/or noncompleteness of $\succsim$ combined with that of $\succsim_{i}$ may obscure some features of models involving traces on differences. For the sake of completeness, we sum up a few useful observations in the following proposition.

Proposition 5.12 (Properties of models using differences). Let $\succsim$ be a binary relation on $X=\prod_{i=1}^{n} X_{i}$ and $J \subseteq N$.

(1) If $\succsim$ satisfies model (D5) then: $\left[x_{i} \succ_{i} y_{i}\right.$ for all $\left.i \in J \subseteq N\right] \Rightarrow \operatorname{Not}\left[y_{J}{ }_{J}\right.$ $\left.x_{J}\right]$.

(2) If $\succsim$ satisfies model (D6) then:

- $\succsim_{i}$ is complete,

- $\left[x_{i} \succ_{i} y_{i}\right.$ for all $\left.i \in J \subseteq N\right] \Rightarrow\left[x_{J} \succ_{J} y_{J}\right]$.

(3) If $\succsim$ satisfies model (D11) then:

- $\left[x_{i} \succsim_{i} y_{i}\right.$ for all $\left.i \in J \subseteq N\right] \Rightarrow\left[x_{J} \succsim_{J} y_{J}\right]$,

- $\left[x_{i} \succsim_{i} y_{i}\right.$ for all $i \in J \subseteq N, x_{j} \succ_{j} y_{j}$, for some $\left.j \in J\right] \Rightarrow\left[x_{J} \succ_{J} y_{J}\right]$.

PROOF. Part 1. Using obvious notation, $x_{i} \succ_{i} y_{i}$ implies $N o t\left[y_{i} \succsim_{i} x_{i}\right]$ so that $G\left(p_{i}\left(y_{i}, x_{i}\right), \mathbf{0}\right)<0$. Since $G(\mathbf{0}) \geq 0$, we know that $p_{i}\left(y_{i}, x_{i}\right)<0$, using the nondecreasingness of $G$. Select any $j \in J$. Starting from $G\left(p_{j}\left(y_{j}, x_{j}\right), \mathbf{0}\right)$ $<0$, using the nondecreasingness of $G$ and the fact that $p_{i}\left(y_{i}, x_{i}\right)<0$, for all $i \in J$, we obtain $G\left(\left(p_{j}\left(y_{j}, x_{j}\right)\right)_{i \in J}, \mathbf{0}\right)<0$. This implies $N o t\left[y_{J} \succsim_{J} x_{J}\right]$.

Part 2. $\operatorname{Not}\left[x_{i} \succsim_{i} y_{i}\right]$ and $\operatorname{Not}\left[y_{i} \succsim_{i} x_{i}\right] \operatorname{imply} G\left(p_{i}\left(x_{i}, y_{i}\right), \mathbf{0}\right)<0$ and $G\left(p_{i}\left(y_{i}\right.\right.$, 
$\left.\left.x_{i}\right), \mathbf{0}\right)<0$. Since $G(\mathbf{0}) \geq 0$ and $G$ is nondecreasing, this implies $p_{i}\left(x_{i}, y_{i}\right)<0$ and $p_{i}\left(y_{i}, x_{i}\right)<0$, which contradicts the skew symmetry of $p_{i}$. Hence $\succsim_{i}$ is complete.

Observe that $x_{i} \succ_{i} y_{i}$ is equivalent to $G\left(p_{i}\left(x_{i}, y_{i}\right), \mathbf{0}\right) \geq 0$ and $G\left(p_{i}\left(y_{i}, x_{i}\right), \mathbf{0}\right)$ $<0$. Since $G(\mathbf{0}) \geq 0$, we know that $p_{i}\left(y_{i}, x_{i}\right)<0$, using the nondecreasingness of $G$. The skew symmetry of $p_{i}$ implies $p_{i}\left(x_{i}, y_{i}\right)>0>p_{i}\left(y_{i}, x_{i}\right)$ and the desired property easily follows using the nondecreasingness of $G$.

Part 3. Since $G$ is increasing and odd, we have $x_{i} \succsim_{i} y_{i} \Leftrightarrow p_{i}\left(x_{i}, y_{i}\right) \geq 0$. The desired properties easily follow from the increasingness of $G$ and $G(\mathbf{0})=0$.

Except for model (D11), the monotonicity properties of our models linking $\succsim$ and $\succsim_{i}$ may seem disappointing. Such properties should however be analyzed keeping in mind that we are dealing with possibly nontransitive and/or incomplete preferences. In such a framework, some "obvious properties" may not always be desirable. For example, when the relations $\sim_{i}$ are not transitive, it may not be reasonable to impose that:

$$
\left[x_{i} \sim_{i} y_{i} \text { for all } i \in J\right] \Rightarrow\left[x_{J} \sim_{J} y_{J}\right],
$$

which would forbid any interaction between separately non-noticeable differences on each attribute (on this point see Gilboa and Lapson (1995) or Pirlot and Vincke (1997)). Clearly, if nice monotonicity properties are looked for, one should use traces (see lemmas 3.7 and 5.5).

Remark 5.13 (Interpretation of $T C$ ). It is not difficult to show that, when $\succsim$ is complete, $\left[R C 1, R C 2\right.$ and $\left(x \sim y\right.$ and $\left(z_{i}, w_{i}\right) \succ_{i}^{* *}\left(x_{i}, y_{i}\right) \Rightarrow\left(z_{i}, x_{-i}\right) \succ$ $\left.\left.\left(w_{i}, y_{-i}\right)\right)\right] \Leftrightarrow T C$. This offers an additional interpretation of $T C$ and shows that the only difference between $(D 11)$ and $(D 7)$ is the possible failure in $(D 7)$ of "strict monotonicity" with respect to $\succ_{i}^{* *}$ for pairs such that $x \sim y$.

Remark 5.14 (Uniqueness). As in section 4.3, the uniqueness properties of the representations exhibited in theorem 5.7 are very weak. Again, numerical representations are only used here as guidelines to investigate the consequences of some particular conditions on $\succsim$ and not as a basis to derive assessment procedures.

Let us analyze the uniqueness properties of model $(D 8)$. Our proof shows that it is always possible to use functions $p_{i}$ such that:

$$
\left(x_{i}, y_{i}\right) \succsim_{i}^{*}\left(z_{i}, w_{i}\right) \Leftrightarrow p_{i}\left(x_{i}, y_{i}\right) \geq p_{i}\left(z_{i}, w_{i}\right) .
$$

This could be called a regular representation of model $(D 8)$. From the proof of theorem 5.7, it is clear that any $p_{i}$ satisfying (5.7) may be used, i.e. we may 
apply, independently on each attribute, any increasing transformation to the functions $p_{i}$ without altering the representation.

Other choices for $p_{i}$ are possible however. Let us show that any function $p_{i}$ such that:

$$
\left(x_{i}, y_{i}\right) \succ_{i}^{*}\left(z_{i}, w_{i}\right) \Rightarrow p_{i}\left(x_{i}, y_{i}\right)>p_{i}\left(z_{i}, w_{i}\right),
$$

can be used in a representation of model (D8).

The necessity of (5.8) is clear since $\left(x_{i}, y_{i}\right) \succ_{i}^{*}\left(z_{i}, w_{i}\right)$ implies $\left(x_{i}, a_{-i}\right) \succsim$ $\left(y_{i}, b_{-i}\right)$ and $\operatorname{Not}\left[\left(z_{i}, a_{-i}\right) \succsim\left(w_{i}, b_{-i}\right)\right]$, for some $a_{-i}, b_{-i} \in X_{-i}$. Using the increasingness of $G$ in model $(D 8)$ this implies $p_{i}\left(x_{i}, y_{i}\right)>p_{i}\left(z_{i}, w_{i}\right)$.

Conversely, it is clear that if $p_{i}$ satisfies (5.8) then

$$
p_{i}\left(x_{i}, y_{i}\right)=p_{i}\left(z_{i}, w_{i}\right) \Rightarrow\left(x_{i}, y_{i}\right) \sim_{i}^{*}\left(z_{i}, w_{i}\right)
$$

so that defining $G$ using (5.5) as in the proof of theorem 5.7 leads to a welldefined function being increasing in its arguments.

It should be noted that any function $f$ (resp. $g$ ) from $\mathbb{R}^{n}$ into $[0,+\infty$ ) (resp. $(-\infty, 0))$ that is increasing in all arguments when restricted to $\prod_{i=1}^{n} p_{i}\left(X_{i}^{2}\right)$ may be used in order to define $G$ letting:

$$
G\left(\left[p_{i}\left(x_{i}, y_{i}\right)\right]\right)= \begin{cases}f\left(\left[p_{i}\left(x_{i}, y_{i}\right)\right]\right) & \text { if } x \succsim y \\ g\left(\left[p_{i}\left(x_{i}, y_{i}\right)\right]\right) & \text { otherwise. }\end{cases}
$$

It is furthermore clear that only such functions may be used.

We have therefore described the set of all possible numerical representations in model $(D 8)$. We shall need below the exact statement of the degrees of freedom we have for choosing the functions $p_{i}$ in our models. This is summarized in the next lemma.

\section{Lemma 5.15 (Uniqueness of $p_{i}$ ).}

(1) Let $\succsim$ satisfy model (D8). A real-valued function $p_{i}$ on $X_{i}^{2}$ may be used in the representation of $\succsim$ in model (D8) iff

$$
\left(z_{i}, w_{i}\right) \succ_{i}^{*}\left(x_{i}, y_{i}\right) \Rightarrow p_{i}\left(z_{i}, w_{i}\right)>p_{i}\left(x_{i}, y_{i}\right)
$$

(2) Let $\succsim$ satisfy model (D9). A real-valued function $p_{i}$ on $X_{i}^{2}$ may be used in the representation of $\succsim$ in model (D9) iff

$$
\begin{gathered}
p_{i}\left(x_{i}, x_{i}\right)=0 \text { and } \\
\left(z_{i}, w_{i}\right) \succ_{i}^{* *}\left(x_{i}, y_{i}\right) \Rightarrow p_{i}\left(z_{i}, w_{i}\right)>p_{i}\left(x_{i}, y_{i}\right) .
\end{gathered}
$$


(3) Let $\succsim$ satisfy model (D7) or (D10). A real-valued function $p_{i}$ on $X_{i}^{2}$ may be used in the representation of $\succsim$ in model (D7) or (D10) iff

$$
\begin{gathered}
p_{i}\left(x_{i}, y_{i}\right)=-p_{i}\left(y_{i}, x_{i}\right) \text { and } \\
\left(z_{i}, w_{i}\right) \succ_{i}^{* *}\left(x_{i}, y_{i}\right) \Rightarrow p_{i}\left(z_{i}, w_{i}\right)>p_{i}\left(x_{i}, y_{i}\right) .
\end{gathered}
$$

(4) Let $\succsim$ satisfy model (D11). A real-valued function $p_{i}$ on $X_{i}^{2}$ may be used in the representation of $\succsim$ in model (D11) iff

$$
\begin{aligned}
& p_{i}\left(x_{i}, y_{i}\right)=-p_{i}\left(y_{i}, x_{i}\right), \\
& \left(z_{i}, w_{i}\right) \succ_{i}^{* *}\left(x_{i}, y_{i}\right) \Rightarrow p_{i}\left(z_{i}, w_{i}\right)>p_{i}\left(x_{i}, y_{i}\right) \text { and } \\
& \left.\begin{array}{l}
\left(z_{i}, w_{i}\right) \sim_{i}^{* *}\left(x_{i}, y_{i}\right) \text { and } \\
\exists a_{-i}, b_{-i} \in X_{-i} \text { s.t. }\left(x_{i}, a_{-i}\right) \sim\left(y_{i}, b_{-i}\right)
\end{array}\right\} \Rightarrow p_{i}\left(z_{i}, w_{i}\right)=p_{i}\left(x_{i}, y_{i}\right) .
\end{aligned}
$$

PROOF. The observations preceding the statement of the lemma prove part 1. The proof of parts 2 and 3 follows from that of part 1.

Part 4. The necessity of (5.16) and (5.17) is clear. Suppose that (5.18) is violated. One would then have $\left(z_{i}, w_{i}\right) \sim_{i}^{* *}\left(x_{i}, y_{i}\right),\left(x_{i}, a_{-i}\right) \sim\left(y_{i}, b_{-i}\right)$ for some $a_{-i}, b_{-i} \in X_{-i}$ and $p_{i}\left(z_{i}, w_{i}\right) \neq p_{i}\left(x_{i}, y_{i}\right)$. Since $G$ is strictly increasing, $G\left(p_{i}\left(z_{i}, w_{i}\right),\left(p_{j}\left(a_{j}, b_{j}\right)\right)_{j \neq i}\right) \neq 0$ while $\left(z_{i}, a_{-i}\right) \sim\left(w_{i}, b_{-i}\right)$, a contradiction.

Sufficiency. Consider, on each $i \in N$, any function $p_{i}$ satisfying (5.16), (5.17) and (5.18) and define $G$ as in (5.6). The well-definedness of $G$ follows from (5.16) and (3.9) since $p_{i}\left(z_{i}, w_{i}\right)=p_{i}\left(x_{i}, y_{i}\right) \Rightarrow\left(z_{i}, w_{i}\right) \sim_{i}^{* *}\left(x_{i}, y_{i}\right)$. For proving increasingness, suppose $p_{i}\left(z_{i}, w_{i}\right)>p_{i}\left(x_{i}, y_{i}\right)$. This implies that $\left(z_{i}, w_{i}\right) \succsim_{i}^{* *}$ $\left(x_{i}, y_{i}\right)$. If $x \succ y,(3.8)$ implies $\left(z_{i}, x_{-i}\right) \succ\left(w_{i}, y_{-i}\right)$ and the conclusion follows from the definition of $G$. If $x \sim y$ we have $G\left(\left[p_{i}\left(x_{i}, y_{i}\right)\right]\right)=0$. Consider two cases. If $\left(z_{i}, w_{i}\right) \succ_{i}^{* *}\left(x_{i}, y_{i}\right)$, then part 2 of lemma 5.5 implies $\left(z_{i}, x_{-i}\right) \succ$ $\left(w_{i}, y_{-i}\right)$ and the conclusion follows from the definition of $G$. If $\left(z_{i}, w_{i}\right) \sim_{i}^{* *}$ $\left(x_{i}, y_{i}\right)$ then, by $(3.9)$ we obtain $\left(z_{i}, x_{-i}\right) \sim\left(w_{i}, y_{-i}\right)$, violating (5.18). Finally, the case $\operatorname{Not}[x \succsim y]$ is dealt with like as in the proof of theorem 5.7. This completes the proof.

Remark 5.16 ( $n=2$ case). It is easy to see that $R C 1_{i}$ amounts to defining a biorder (see Doignon et al., 1984; Ducamp and Falmagne, 1969) between the sets $X_{i}^{2}$ and $X_{-i}^{2}$. Therefore $R C 1_{i}$ on its own implies, when $X$ is finite or countably infinite, the existence of two real-valued functions $p_{i}$ and $P_{-i}$ respectively on $X_{i}^{2}$ and $X_{-i}^{2}$ such that, for all $x, y \in X, x \succsim y$ iff $p_{i}\left(x_{i}, y_{i}\right)+$ $P_{-i}\left(x_{-i}, y_{-i}\right) \geq 0$ (see Ducamp and Falmagne, 1969, Proposition 3).

Therefore models using traces on differences closely relate to ordinal measurement when $n=2$. 
In a similar vein, Bouyssou (1986, Theorem 1) noted an interesting implication of $T C_{i}$ on its own. When $X$ is countable, $T C_{i}$ implies the existence of two realvalued skew symmetric functions $p_{i}$ and $P_{-i}$ respectively on $X_{i}^{2}$ and $X_{-i}^{2}$ such that, for all $x, y \in X, x \succsim y \Leftrightarrow p_{i}\left(x_{i}, y_{i}\right)+P_{-i}\left(x_{-i}, y_{-i}\right) \geq 0$. This result can easily be extended to sets of arbitrary cardinality (see remark 5.18). When $n=2$ this offers an alternative to Fishburn (1991a, theorem B).

Remark 5.17 (Extensions to subsets). The obvious extension of $R C 1$ to subsets of attributes is the main necessary condition used by Vind (1991) together with topological assumptions on $X$ to axiomatize a model such that:

$$
x \succsim y \Leftrightarrow \sum_{i=1}^{n} p_{i}\left(x_{i}, y_{i}\right) \geq 0
$$

with $p_{i}\left(x_{i}, x_{i}\right)=0$.

Similarly, it is easy to see that the extension of $T C$ to subsets of attributes is necessary for a model of type (5.19) with all $p_{i}$ 's being skew symmetric. A complete axiomatic treatment of this model may be found in Fishburn (1990a,b, 1991a).

It should be noted that our use here of a function $G$ instead of a sum allows to considerably simplify our analysis. Clearly as soon as additivity is required, axioms involve either a denumerable scheme of conditions guaranteeing the existence of solutions to a set of linear equations or a limited number of conditions together with unnecessary structural assumptions on the set of alternatives (e.g. solvability). We refer to Bouyssou and Pirlot (2002d) for a thorough comparison between models involving traces on differences and their additive specializations as well as an evaluation of the contribution of theorem 5.7 to the general theory of conjoint measurement.

Remark 5.18 (The general case). The results in this section can easily be extended to cover the general case. This requires the addition of, necessary, conditions guaranteeing that the equivalences and weak orders encountered in this section have a numerical representation.

The main results in this section are summarized in table 6 and figure 4 .

\section{Models using marginal traces on levels and on differences ${ }^{5}$}

This section studies models based on traces on differences in which the magnitude of differences may be modelled through traces on levels. We have already

$\overline{5}$ This section is based on Bouyssou and Pirlot (2002a) 
Table 6

Main results using traces on differences

\begin{tabular}{|c|c|c|}
\hline Models & Definition & Conditions \\
\hline$(D 0)$ & $x \succsim y \Leftrightarrow G\left(\left[p_{i}\left(x_{i}, y_{i}\right)\right]\right) \geq 0$ & $\varnothing$ \\
\hline$(D 1)$ & $\begin{array}{c}(D 0) \text { with } p_{i}\left(x_{i}, x_{i}\right)=0 \\
(D 0 \ldots \\
\end{array}$ & \\
\hline $\begin{array}{c}\mathbb{1} \\
(D 2)\end{array}$ & $(D 0)$ with $p_{i}$ skew symmetric & ind. \\
\hline$(D 3)$ & $\begin{array}{c}(D 0) \text { with } p_{i} \text { skew symmetric } \\
\text { and } G \text { odd }\end{array}$ & cpl., ind. \\
\hline$(D 8) \Leftrightarrow(D 4)$ & $\begin{array}{l}(D 0) \text { with } G(I /) \\
\end{array}$ & $R C 1$ \\
\hline$(D 9) \Leftrightarrow(D 5)$ & $(D 1)$ with $G(\nearrow \nearrow)$ & $R C 1$, ind. \\
\hline$(D 10) \Leftrightarrow(D 6)$ & $(D 2)$ with $G(\nearrow \nearrow)$ & $R C 12$ \\
\hline$(D 7)$ & $\begin{array}{c}(D 3) \text { with } G(\nearrow) \\
(\nearrow, \ldots \ldots \ldots \ldots \ldots\end{array}$ & cpl., $R C 12$ \\
\hline$(D 11)$ & (D3) with $G(\nearrow \nearrow)$ & cpl., $T C$ \\
\hline
\end{tabular}

Figure 4. Implication between models resulting from theorem 5.7

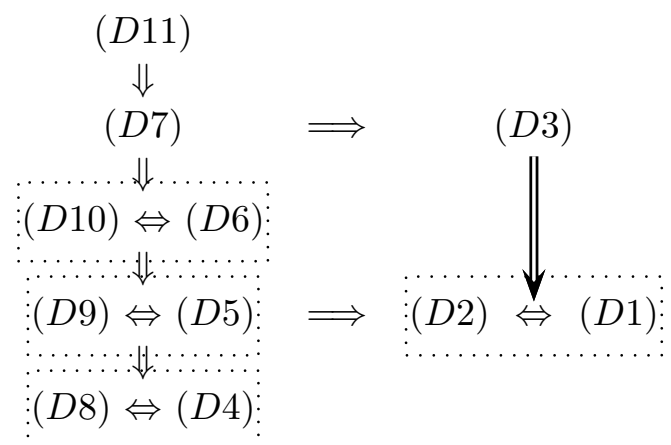

$$
\text { All models imply }(D 0)
$$

encountered the trivial model $(L 0 D 0)$ in which:

$$
x \succsim y \Leftrightarrow H\left(\left[\phi_{i}\left(u_{i}\left(x_{i}\right), u_{i}\left(y_{i}\right)\right)\right]\right) \geq 0,
$$

This model may be seen as a particular case of model $(D 0)$ in which the term $p_{i}\left(x_{i}, y_{i}\right)$ is substituted with the term $\phi_{i}\left(u_{i}\left(x_{i}\right), u_{i}\left(y_{i}\right)\right)$. Alternatively it may be seen as a generalization of the additive difference model (1.5) replacing addition and subtraction by more general functions.

To each of the 12 models $(D 0)$ to $(D 11)$ studied in section 5 corresponds a 
model in which the term $p_{i}\left(x_{i}, y_{i}\right)$ is substituted with $\phi_{i}\left(u_{i}\left(x_{i}\right), u_{i}\left(y_{i}\right)\right)$. This defines models $(L 0 D 0)$ to $(L 0 D 11)$.

In order to bring the functions $\phi_{i}$ "closer" to a subtraction, we envisage two variants of each of these models. In the first one, we impose that $\phi_{i}$ are nondecreasing in their first argument and nonincreasing in their second argument. This defines models $(L 1 D 0)$ to $(L 1 D 11)$. In the other variant we impose that $\phi_{i}$ are increasing in their first argument and decreasing in their second argument. This defines models $(L 2 D 0)$ to $(L 2 D 11)$. The definition of all these models is summarized in table 7 . We have thus defined a total of $3 \times 12=36$ models involving differences and levels. We study them in this section.

Table 7

Models involving traces on levels and on differences

$$
\begin{aligned}
& (L 0 D 0) \quad x \succsim y \Leftrightarrow H\left(\left[\phi_{i}\left(u_{i}\left(x_{i}\right), u_{i}\left(y_{i}\right)\right)\right]\right) \geq 0 \\
& (L 0 D 1) \quad(L 0 D 0) \text { with } \phi_{i}\left(u\left(x_{i}\right), u_{i}\left(x_{i}\right)\right)=0 \\
& (L 0 D 2) \quad(L 0 D 1) \text { with } \phi_{i} \text { skew symmetric } \\
& \text { (LOD3) (LOD2) with } \mathrm{H} \text { odd } \\
& \text { (L0D4) } \quad(L 0 D 0) \text { with } H \text { nondecreasing } \\
& (L 0 D 5) \quad(L 0 D 0) \text { with } H \text { increasing } \\
& \text { (L0D6) } \quad(L 0 D 1) \text { with } H \text { nondecreasing } \\
& (L 0 D 7) \quad(L 0 D 1) \text { with } H \text { increasing } \\
& \text { (L0D8) } \quad(L 0 D 2) \text { with } H \text { nondecreasing } \\
& (L 0 D 9) \quad(L 0 D 2) \text { with } H \text { increasing } \\
& (L 0 D 10) \quad(L 0 D 3) \text { with } H \text { nondecreasing } \\
& \text { (L0D11) } \quad(L 0 D 3) \text { with } H \text { increasing }
\end{aligned}
$$

Models $(L 1 D x)$ correspond to models $(L 0 D x)$ with $\phi_{i}(\nearrow, \searrow)$ Models $(L 2 D x)$ correspond to models $(L 0 D x)$ with $\phi_{i}(\nearrow \nearrow, \searrow \backslash)$

\subsection{Models (L0D1) to (L0D11)}

These 12 models $(L 0 D 0)$ to $(L 0 D 11)$ correspond to models $(D 0)$ to $(D 11)$ involving differences in which the term $p_{i}\left(x_{i}, y_{i}\right)$ is substituted with the term $\phi_{i}\left(u_{i}\left(x_{i}\right), u_{i}\left(y_{i}\right)\right)$ with no monotonicity property required for the functions $\phi_{i}$. These models are easily analyzed using the following elementary observation.

Lemma 6.1 (Numerical representation of a weak order on pairs). Let $\succsim^{A}$ be a weak order on a countable set $A^{2}$. Consider any real valued function 
$f$ on $A^{2}$ representing $\succsim^{A}$, i.e. such that, for all $a, b, c, d \in A$,

$$
(a, b) \succsim^{A}(c, d) \Leftrightarrow f(a, b) \geq f(c, d) .
$$

There is a real-valued function $u$ on $A$ and a real-valued function $g$ on $u(A) \times$ $u(A)$ such that, for all $a, b \in A$,

$$
f(a, b)=g(u(a), u(b)) .
$$

PROOF. Define the binary relation $E$ on $A$ letting, for all $a, b \in A$,

$$
a E b \Leftrightarrow(a, c) \sim^{A}(b, c), \text { and }(c, b) \sim^{A}(c, a) \text {, for all } c \in A,
$$

where $\sim^{A}$ denotes the symmetric part of $\succsim^{A}$. Since $\sim^{A}$ is an equivalence, it is easy to show that $E$ is an equivalence. Therefore, since $A$ is countable, there is a real-valued function $u$ on $A$ such that, for all $a, b \in A$,

$$
a E b \Leftrightarrow u(a)=u(b) .
$$

Take any function $f$ such that (6.1) holds and define $g$ on $u(A) \times u(A)$ letting, for all $a, b \in A, g(u(a), u(b))=f(a, b)$. We have to show that $g$ is well-defined, i.e. that $u(a)=u(c)$ and $u(b)=u(d)$ imply $f(a, b)=f(c, d)$. By construction, we have $a E c$ and $b E d$. This implies $(a, \ell) \sim^{A}(c, \ell)$ and $\left(\ell^{\prime}, b\right) \sim^{A}\left(\ell^{\prime}, d\right)$, for all $\ell, \ell^{\prime} \in A$. Taking $\ell=b$ and $\ell^{\prime}=c$ implies $(a, b) \sim^{A}(c, b)$ and $(c, b) \sim^{A}(c, d)$. Using the transitivity of $\sim^{A}$ we obtain $(a, b) \sim^{A}(c, d)$ so that $f(a, b)=f(c, d)$, as required.

Theorem 6.2 (Models (L0D0) to (L0D11)). Models (L0D0) to (L0D11) are respectively equivalent to models (D0) to (D11).

PROOF. Consider any representation of models (D0) to (D11) and apply lemma 6.1 to the weak order on $X_{i}^{2}$ induced by $p_{i}$.

Models $(L 0 D 0)$ to $(L 0 D 11)$ are therefore nothing more than a different presentation of models $(D 0)$ to $(D 11)$. Clearly, the equivalences between models $(D 0)$ to $(D 11)$ noted in corollary 5.8 carry over to models $(L 0 D 0)$ to $(L 0 D 11)$. The relations between these models is therefore given by figure 4 replacing $D k$ by $L 0 D k$, for $k=0,1, \ldots, 11$.

\subsection{Models (L1D0)-(L1D3), (L2D0)-(L2D3)}

We first take up the case of models $(L 1 D 0)$ to $(L 1 D 3)$ and $(L 2 D 0)$ to $(L 2 D 3)$ in which $H$ has no particular monotonicity properties. Substituting $p_{i}\left(x_{i}, y_{i}\right)$ 
with $\phi_{i}\left(u_{i}\left(x_{i}\right), u_{i}\left(y_{i}\right)\right), \phi_{i}$ being nondecreasing in its first argument and nonincreasing in its second argument is likely to have little impact since the monotonicity properties of $\phi_{i}$ can be "absorbed" by $H$. A similar reasoning applies if $\phi_{i}$ is supposed to be increasing in its first argument and decreasing in its second argument. As already mentioned in remark 3.10, this is indeed the case.

Theorem 6.3 (Models $(L 1 D 0)-(L 1 D 3)$ and $(L 2 D 0)-(L 2 D 3))$.

(1) $(D 0) \Leftrightarrow(L 1 D 0) \Leftrightarrow(L 2 D 0)$,

(2) $(D 1) \Leftrightarrow(L 1 D 1) \Leftrightarrow(L 2 D 1) \Leftrightarrow(D 2) \Leftrightarrow(L 1 D 2) \Leftrightarrow(L 2 D 2)$,

(3) $(D 3) \Leftrightarrow(L 1 D 3) \Leftrightarrow(L 2 D 3)$.

PROOF. Part 1 . By construction, $(L 2 D 0) \Rightarrow(L 1 D 0) \Rightarrow(D 0)$. We show that $(D 0) \Rightarrow(L 2 D 0)$.

Consider any one-to-one correspondence $u_{i}$ between $X_{i}$ and a subset of the set of integers $\geq 2$. Define $\phi_{i}$ letting, for all $x_{i}, y_{i} \in X_{i}$,

$$
\phi_{i}\left(u_{i}\left(x_{i}\right), u_{i}\left(y_{i}\right)\right)=u_{i}\left(x_{i}\right)+\frac{1}{u_{i}\left(y_{i}\right)} .
$$

By construction, $\phi_{i}$ is increasing in its first argument and decreasing in its second argument. Observe that

$$
\phi_{i}\left(u_{i}\left(x_{i}\right), u_{i}\left(y_{i}\right)\right)=\phi_{i}\left(u_{i}\left(z_{i}\right), u_{i}\left(w_{i}\right)\right) \Rightarrow x_{i}=z_{i} \text { and } y_{i}=w_{i}
$$

Define $H$ on $\prod_{i=1}^{n} \phi_{i}\left(u_{i}\left(X_{i}\right), u_{i}\left(X_{i}\right)\right)$ letting:

$$
H\left(\left[\phi_{i}\left(u_{i}\left(x_{i}\right), u_{i}\left(y_{i}\right)\right)\right]\right)=\left\{\begin{array}{l}
+1 \text { if } x \succsim y, \\
-1 \text { otherwise. }
\end{array}\right.
$$

We have to show that $H$ is well-defined. This is obvious in view of (6.6).

Part 2. We know from corollary 5.8 that $(D 2) \Leftrightarrow(D 1)$. By construction $(L 2 D 2)$ $\Rightarrow(L 1 D 2) \Rightarrow(D 2)$ and $(L 2 D 1) \Rightarrow(L 1 D 1) \Rightarrow(D 1)$. We show that $(D 1) \Rightarrow$ $(L 2 D 2)$.

Consider on each $X_{i}$ any function $\phi_{i}$ satisfying (6.5) and define $\varphi_{i}$ letting, for all $x_{i}, y_{i} \in X_{i}$,

$$
\varphi_{i}\left(u_{i}\left(x_{i}\right), u_{i}\left(y_{i}\right)\right)=\phi_{i}\left(u_{i}\left(x_{i}\right), u_{i}\left(y_{i}\right)\right)-\phi_{i}\left(u_{i}\left(y_{i}\right), u_{i}\left(x_{i}\right)\right) .
$$

By construction, $\varphi_{i}$ is skew symmetric, increasing in its first argument and decreasing in its second argument. We clearly have:

$$
\varphi_{i}\left(u_{i}\left(x_{i}\right), u_{i}\left(y_{i}\right)\right)=\varphi_{i}\left(u_{i}\left(z_{i}\right), u_{i}\left(w_{i}\right)\right) \Rightarrow\left\{\begin{array}{l}
x_{i}=z_{i} \text { and } y_{i}=w_{i} \\
\text { or } \\
x_{i}=y_{i} \text { and } z_{i}=w_{i} .
\end{array}\right.
$$


Define $H$ on $\prod_{i=1}^{n} \varphi_{i}\left(u_{i}\left(X_{i}\right), u_{i}\left(X_{i}\right)\right)$ letting:

$$
H\left(\left[\varphi_{i}\left(u_{i}\left(x_{i}\right), u_{i}\left(y_{i}\right)\right)\right]\right)=\left\{\begin{array}{l}
+1 \text { if } x \succsim y, \\
-1 \text { otherwise }
\end{array}\right.
$$

Since model $(D 1)$ holds, we know that $\succsim$ is independent so that $\left(x_{i}, x_{i}\right) \sim_{i}^{*}$ $\left(y_{i}, y_{i}\right)$. In view of $(6.9)$, it is then clear that $H$ is well-defined.

Part 3. By construction, $(L 2 D 3) \Rightarrow(L 1 D 3) \Rightarrow(D 3)$. We show that $(D 3)$ $\Rightarrow(L 2 D 3)$. Define, on each $i \in N$, a function $\varphi_{i}$ using (6.8). Define $H$ on $\prod_{i=1}^{n} \varphi_{i}\left(u_{i}\left(X_{i}\right), u_{i}\left(X_{i}\right)\right)$ letting:

$$
H\left(\left[\varphi_{i}\left(u_{i}\left(x_{i}\right), u_{i}\left(y_{i}\right)\right)\right]\right)=\left\{\begin{array}{rc}
+1 & \text { if } x \succ y \\
0 & \text { if } x \sim y \\
-1 & \text { otherwise }
\end{array}\right.
$$

Since $\succsim$ is complete, $H$ is odd. Its well-definedness is proved as in part 2 .

\subsection{Models (L1D4) to (L1D11)}

These eight models are the counterparts of models $(D 4)$ to $(D 11)$ in which the term $p_{i}\left(x_{i}, y_{i}\right)$ is substituted with the term $\phi_{i}\left(u_{i}\left(x_{i}\right), u_{i}\left(y_{i}\right)\right), \phi_{i}$ being nondecreasing in its first argument and nonincreasing in its second argument. In all models $(D 4)$ to $(D 11)$, the function $G$ is nondecreasing in all its arguments. Therefore, it is clear that imposing that $\phi_{i}$ is nondecreasing in its first argument and nonincreasing in its second argument is no more innocuous. The conditions that were used to analyze models using traces on levels reappear here. The least constrained of the models considered here, (L1D4), already implies all of these conditions.

Lemma 6.4 (Necessity of $A C 1, A C 2$ and $A C 3$ ). If $\succsim$ has a representation in model (L1D4) then it satisfies $A C 1, A C 2$ and $A C 3$.

PROOF. In model ( $L 1 D 4)$, we have:

$$
x \succsim y \Leftrightarrow H\left(\left[\phi_{i}\left(u_{i}\left(x_{i}\right), u_{i}\left(y_{i}\right)\right)\right]\right) \geq 0
$$

with $H$ being nondecreasing in all its arguments and all $\phi_{i}$ being nondecreasing in their first argument and nonincreasing in their second argument. The proof that $A C 1, A C 2$ and $A C 3$ hold easily follows from the numerical representation. We show that $A C 1$ holds. The premise of $A C 1_{i}$ yields in terms of model 
$(L 1 D 4)$ :

$$
\begin{aligned}
& H\left(\phi_{i}\left(u_{i}\left(x_{i}\right), u_{i}\left(y_{i}\right)\right),\left(\phi_{j}\left(u_{j}\left(x_{j}\right), u_{j}\left(y_{j}\right)\right)\right)_{j \neq i}\right) \geq 0 \text { and } \\
& H\left(\phi_{i}\left(u_{i}\left(z_{i}\right), u_{i}\left(w_{i}\right)\right),\left(\phi_{j}\left(u_{j}\left(z_{j}\right), u_{j}\left(w_{j}\right)\right)\right)_{j \neq i}\right) \geq 0 .
\end{aligned}
$$

We have either $u_{i}\left(z_{i}\right) \geq u_{i}\left(x_{i}\right)$ or $u_{i}\left(x_{i}\right) \geq u_{i}\left(z_{i}\right)$. In the first case, the monotonicity of $H$ and $\phi_{i}$, implies:

$$
H\left(\phi_{i}\left(u_{i}\left(z_{i}\right), u_{i}\left(y_{i}\right)\right),\left(\phi_{j}\left(u_{j}\left(x_{j}\right), u_{j}\left(y_{j}\right)\right)\right)_{j \neq i}\right) \geq 0 .
$$

In the second case, we have:

$$
H\left(\phi_{i}\left(u_{i}\left(x_{i}\right), u_{i}\left(w_{i}\right)\right),\left(\phi_{j}\left(u_{j}\left(z_{j}\right), u_{j}\left(w_{j}\right)\right)\right)_{j \neq i}\right) \geq 0 .
$$

Hence, $A C 1_{i}$ holds. The proof for $A C 2_{i}$ and $A C 3_{i}$ is similar.

As was the case in section 6.1, a simple lemma on the numerical representation of a weak order on ordered pairs will allow us to analyze all our models. This will require some new vocabulary however.

Definition 6.5 (Strong linearity). Let $\succsim^{A}$ be a binary relation on a set $A^{2}$. We say that:

(1) $\succsim^{A}$ is right-linear iff $\left[N o t\left[(b, c) \succsim^{A}(a, c)\right] \Rightarrow(a, d) \succsim^{A}(b, d)\right]$,

(2) $\succsim^{A}$ is left-linear iff $\left[N o t\left[(c, a) \succsim^{A}(c, b)\right] \Rightarrow(d, b) \succsim^{A}(d, a)\right]$,

(3) $\succsim^{A}$ is strongly linear iff $\left[\operatorname{Not}\left[(b, c) \succsim^{A}(a, c)\right]\right.$ or $\left.\operatorname{Not}\left[(c, a) \succsim^{A}(c, b)\right]\right] \Rightarrow$ $\left[(a, d) \succsim^{A}(b, d)\right.$ and $\left.(d, b) \succsim^{A}(d, a)\right]$,

for all $a, b, c, d \in A$

Lemma 6.6 (Numerical representation of a weak order on pairs). Let $\succsim^{A}$ be a weak order on a countable set $A^{2}$. Let $f$ be any real-valued function on $A^{2}$ such that, for all $a, b, c, d \in A$,

$$
(a, b) \succsim^{A}(c, d) \Leftrightarrow f(a, b) \geq f(c, d) .
$$

There is a real-valued function $u$ on $A$ and a real-valued function $g$ on $u(A) \times$ $u(A)$ nondecreasing in its first argument, nonincreasing in its second argument, such that for all $a, b, c, d \in A$,

$$
f(a, b)=g(u(a), u(b))
$$

iff $\succsim^{A}$ is strongly linear.

PROOF. Necessity of strong linearity is easily shown. Suppose that $\operatorname{Not}\left[(b, c) \succsim^{A}\right.$ $(a, c)]$ or $\operatorname{Not}\left[(c, a) \succsim^{A}(c, b)\right]$. This implies $g(u(b), u(c))<g(u(a), u(c))$ or 
$g(u(c), u(a))<g(u(c), u(b))$. In either case, the monotonicity properties of $g$ imply $u(a)>u(b)$. Starting now from $g(u(b), u(d))$ and using the nondecreasingness of $g$ in its first argument, we obtain $g(u(a), u(d)) \geq g(u(b), u(d))$ and, hence, $(a, d) \succsim^{A}(b, d)$. The proof that $(d, b) \succsim^{A}(d, a)$ holds is similar.

Sufficiency. Define the binary relation $T$ on $A$ letting:

$$
a T b \Leftrightarrow[(a, c) \succsim(b, c) \text { and }(c, b) \succsim(c, a) \text {, for all } c \in A] \text {. }
$$

It is clear that $T$ is reflexive and transitive. An easy proof shows that it is complete if and only if $\succsim$ is strongly linear.

Since $A$ is countable and $T$ is a weak order, there is a real-valued function $u$ on $A$ such that, for all $a, b \in A$,

$$
a T b \Leftrightarrow u(a) \geq u(b) .
$$

Let $f$ be any real-valued function on $A^{2}$ such that (6.12) holds. Define the real-valued function $g$ on $u(A)^{2}$ letting, for all $a, b, c, d \in A$,

$$
g(u(a), u(b))=f(a, b) .
$$

Using the definition of $T$, it is routine to show that $g$ is well-defined, nondecreasing in its first argument and nonincreasing in its second argument.

The following lemma interprets conditions $A C 1, A C 2$ and $A C 3$ in terms of linearity properties of $\succsim_{i}^{*}$.

Lemma $6.7\left(A C 1_{i}, A C 2_{i}, A C 3_{i}\right.$ and strong linearity). Let $\succsim$ be a binary relation on $X=\prod_{i=1}^{n} X_{i}$.

(1) $A C 1_{i}$ holds iff $\succsim_{i}^{*}$ is right linear,

(2) $A C 2_{i}$ holds iff $\succsim_{i}^{*}$ is left linear,

(3) $A C 3_{i}$ holds iff $\left[N o t\left[\left(x_{i}, z_{i}\right) \succsim_{i}^{*}\left(y_{i}, z_{i}\right)\right]\right.$, for some $\left.z_{i} \in X_{i}\right] \Rightarrow\left(w_{i}, x_{i}\right) \succsim_{i}^{*}$ $\left(w_{i}, y_{i}\right)$, for all $w_{i} \in X_{i}$,

(4) $A C 1_{i}, A C 2_{i}$ and $A C 3_{i}$ hold iff $\succsim_{i}^{*}$ is strongly linear iff $\succsim_{i}^{* *}$ is strongly linear.

PROOF. Part 1. We show equivalently that $\operatorname{Not}\left[A C 1_{i}\right]$ iff for some $x_{i}, y_{i}, z_{i}, w_{i} \in$ $X_{i}, \operatorname{Not}\left[\left(z_{i}, y_{i}\right) \succsim_{i}^{*}\left(x_{i}, y_{i}\right)\right]$ and $\operatorname{Not}\left[\left(x_{i}, w_{i}\right) \succsim_{i}^{*}\left(z_{i}, w_{i}\right)\right]$. This last statement means, by definition of $\succsim_{i}^{*}$, that for some $a_{-i}, b_{-i}, c_{-i}, d_{-i} \in X_{-i}$, we have

$$
\begin{aligned}
& {\left[\left(x_{i}, a_{-i}\right) \succsim\left(y_{i}, b_{-i}\right)\right] \text { and } \operatorname{Not}\left[\left(z_{i}, a_{-i}\right) \succsim\left(y_{i}, b_{-i}\right)\right], \text { and }} \\
& {\left[\left(z_{i}, c_{-i}\right) \succsim\left(w_{i}, d_{-i}\right)\right] \text { and } \operatorname{Not}\left[\left(x_{i}, c_{-i}\right) \succsim\left(w_{i}, d_{-i}\right)\right],}
\end{aligned}
$$


which is exactly $\operatorname{Not}\left[A C 1_{i}\right]$. The proof of part 2 is similar.

Part 3. We show that $\operatorname{Not}\left[A C 3_{i}\right] \Leftrightarrow\left[\operatorname{Not}\left[\left(x_{i}, z_{i}\right) \succsim_{i}^{*}\left(y_{i}, z_{i}\right)\right]\right.$ and $\operatorname{Not}\left[\left(w_{i}, x_{i}\right) \succsim_{i}^{*}\right.$ $\left.\left.\left(w_{i}, y_{i}\right)\right]\right]$, for some $z_{i}, w_{i} \in X_{i}$. The last expression means that, for some $a_{-i}, b_{-i}, c_{-i}, d_{-i} \in X_{-i}$, we have

$$
\begin{gathered}
{\left[\left(y_{i}, a_{-i}\right) \succsim\left(z_{i}, b_{-i}\right)\right] \text { and } \operatorname{Not}\left[\left(x_{i}, a_{-i}\right) \succsim\left(z_{i}, b_{-i}\right)\right],} \\
{\left[\left(w_{i}, c_{-i}\right) \succsim\left(y_{i}, d_{-i}\right)\right] \text { and } \operatorname{Not}\left[\left(w_{i}, c_{-i}\right) \succsim\left(x_{i}, d_{-i}\right)\right],}
\end{gathered}
$$

which is exactly $\operatorname{Not}\left[A C 3_{i}\right]$.

Part 4. Combining the equivalences in parts 1, 2 and 3 leads to the equivalence between $A C 1_{i}, A C 2_{i}$ and $A C 3_{i}$ with the strong linearity of $\succsim_{i}^{*}$. By construction this also shows that $\succsim_{i}^{* *}$ is strongly linear.

We are now in position to characterize models $(L 1 D 4)$ to $(L 1 D 11)$

Theorem 6.8. Let $\succsim$ be a binary relation on a countable set $X=\prod_{i=1}^{n} X_{i}$.

(1) Model (L1D8) holds iff $\succsim$ satisfies RC1, AC1, AC2 and $A C 3$.

(2) Model (L1D9) holds iff $\succsim$ is independent and satisfies RC1, AC1, AC2 and $A C 3$.

(3) Model (L1D10) holds iff $\succsim$ satisfies RC1, RC2, AC1, AC2 and AC3.

(4) Model (L1D7) holds iff $\succsim$ is complete and satisfies $R C 1, R C 2, A C 1$, $A C 2$ and $A C 3$.

(5) Model (L1D11) holds iff $\succsim$ is complete and satisfies TC, AC1, AC2 and $A C 3$.

PROOF. The necessity of all parts follows from theorem 5.7 and lemma 6.4.

Observe that in the proof of parts 3 to 7 of theorem 5.7, we have exhibited a regular representation, i.e. a representation in which $p_{i}$ represents $\succsim_{i}^{*}$ in models $(D 8)$ and $(D 9)$ and $\succsim_{i}^{* *}$ in models $(D 10),(D 7)$ and $(D 11)$. The sufficiency of each part therefore follows applying lemma 6.6 to the weak order induced on $X_{i}^{2}$ by the function $p_{i}$ used in the proof of theorem 5.7 .

\section{Corollary 6.9 (Links between models).}

(1) Model (L1D4) and (L1D8) are equivalent.

(2) Model (L1D5) and (L1D9) are equivalent.

(3) Models (L1D6) and (L1D10) are equivalent.

(4) Model (L1D7) $\Rightarrow$ Model (L1D10). 
Remark 6.10 (Independence of axioms). We refer to Bouyssou and Pirlot (2002a) for a complete analysis of the independence of the axioms used in theorem 6.8. It turns out that conditions implying the completeness of marginal traces on levels ( $A C 1, A C 2$ and $A C 3$ ) are completely independent from the conditions implying that marginal traces on differences are complete $(R C 1$ and $R C 2$ ). In order not to multiply examples, we simply show in appendix A.3 that, in the class of complete relations, conditions $T C, A C 1, A C 2$ and $A C 3$ are independent.

\subsection{Models (L2D4) to (L2D11)}

Except for the most constrained model ( $L 2 D 11)$, it turns out that no additional constraint is brought into the picture supposing that $\phi_{i}$ increases in its first arguments and decreases in its second argument. We summarize our results below.

Theorem 6.11. Let $\succsim$ be a binary relation on a countable set $X=\prod_{i=1}^{n} X_{i}$.

(1) Models (L2D8) and (L1D8) are equivalent.

(2) Models (L2D9) and (L1D9) are equivalent.

(3) Models (L2D10) and (L1D10) are equivalent.

(4) Models (L2D7) and (L1D7) are equivalent.

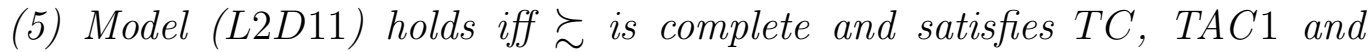
$T A C 2$.

In the proof of theorem 6.8 the strategy was to consider the underlying models involving differences and to factorize the function $p_{i}$ using lemma 6.6 using the fact that it is always possible to build a regular representation. Hence in all the representations used in theorem 6.8 the functions $\phi_{i}$ represent $\succsim_{i}^{*}$ or $\succsim_{i}^{* *}$ depending on the model. Our strategy here is rather different and amounts to exploiting the fact, already stressed, that there is no need for the functions $p_{i}$ in models involving differences to represent $\succsim_{i}^{*}$ or $\succsim_{i}^{* *}$. We use this degree of freedom to modify nondecreasing/nonincreasing functions $\phi_{i}$ into increasing and decreasing functions $\varphi_{i}$.

The following lemma states the conditions under which a function $f$ that is nondecreasing in its first argument and nonincreasing in its second argument, can be appropriately transformed into a strictly monotonic function $g$.

Consider a function $f: U \times U \rightarrow \mathbb{R}$, with $U$ a countable subset of $\mathbb{R}$ and suppose that $f$ is nondecreasing in its first argument and nonincreasing in its second argument. There are two types of situations that may cause the lack of strict monotonicity of $f$. We denote by $S$, the set of values $r$ of $f$ for which 
either there are $a, b, c \in U$ such that:

$$
f(a, c)=f(b, c)=r \text { with } a>b,
$$

or there are $a, c, d \in U$ such that:

$$
f(a, c)=f(a, d)=r \text { with } c>d .
$$

Clearly, $f$ is strictly monotonic iff $S$ is empty.

Lemma 6.12 (Modifying a nondecreasing function). Let $U$ be a countable subset of the $(0,1)$ interval and $f: U \times U \rightarrow \mathbb{R}$ a function that is nondecreasing in its first argument and nonincreasing in its second argument.

There exists a function $g: U \times U \rightarrow \mathbb{R}$ that is increasing in its first argument and decreasing in its second argument and such that, for all $u, v, u^{\prime}, v^{\prime} \in U$,

$$
\begin{aligned}
& \left.\left[f(u, v)>f\left(u^{\prime}, v^{\prime}\right)\right] \Rightarrow[g(u, v))>g\left(u^{\prime}, v^{\prime}\right)\right], \text { and } \\
& \left.\left[f(u, v)=f\left(u^{\prime}, v^{\prime}\right)\right] \Rightarrow[g(u, v))=g\left(u^{\prime}, v^{\prime}\right)\right] \text { iff } f(u, v) \notin S .
\end{aligned}
$$

If, in addition, $f$ vanishes on the diagonal $(f(u, u)=0$, for all $u \in U)$ (resp. is skew symmetric) there exists a function g satisfying (6.17) and (6.18) that vanishes on the diagonal (resp. is skew symmetric).

The proof of this lemma, being rather uninformative, is relegated in appendix B.

Proof of Theorem 6.11 Parts 1 to 4 . We show that model ( $L 2 D 8)$ holds if and only if $R C 1$ and $A C 123$ hold.

The necessity of $R C 1$ and $A C 123$ for model ( $L 2 D 8$ ) is clear, using theorem 6.8. We show sufficiency. Consider a representation of $\succsim$ in model $(L 1 D 8)$. From the proof of theorem 6.8 , we have:

$$
\left(z_{i}, w_{i}\right) \succsim_{i}^{*}\left(x_{i}, y_{i}\right) \Leftrightarrow \phi_{i}\left(u_{i}\left(z_{i}\right), u_{i}\left(w_{i}\right)\right) \geq \phi_{i}\left(u_{i}\left(x_{i}\right), u_{i}\left(y_{i}\right)\right) .
$$

Without loss of generality, we may suppose that $U=u_{i}\left(X_{i}\right)$ is included in $(0,1)$. We may then apply lemma 6.12 to obtain a function $\varphi_{i}$ that is increasing in its first argument and decreasing in its second argument. According to (6.17), we have:

$$
\left(z_{i}, w_{i}\right) \succ_{i}^{*}\left(x_{i}, y_{i}\right) \Rightarrow \varphi_{i}\left(u_{i}\left(z_{i}\right), u_{i}\left(w_{i}\right)\right)>\varphi_{i}\left(u_{i}\left(x_{i}\right), u_{i}\left(y_{i}\right)\right) .
$$

Hence, this function can be used as a basis of the definition of $H$ in view of lemma 5.15. This shows sufficiency. 
Combining theorem 6.8 and lemmas 5.15 and 6.12, the proof for models (L2D9), (L2D10) and (L2D7) is similar.

Part 5. Necessity. In view of theorem 6.8 we only have to show that TAC 1 and TAC2 are necessary. Suppose that $\succsim$ has a representation in model (L2D11). The premise of $T A C 1_{i}$, interpreted in terms of the model, yields three inequalities:

$$
\begin{aligned}
H\left(\phi_{i}\left(u_{i}\left(x_{i}\right), u_{i}\left(y_{i}\right)\right),\left(\phi_{j}\left(u_{j}\left(a_{j}\right), u_{j}\left(y_{j}\right)\right)_{j \neq i}\right)\right. & \geq 0 \\
H\left(\phi_{i}\left(u_{i}\left(y_{i}\right), u_{i}\left(z_{i}\right)\right),\left(\phi_{j}\left(u_{j}\left(y_{j}\right), u_{j}\left(a_{j}\right)\right)_{j \neq i}\right)\right. & \geq 0 \\
H\left(\phi_{i}\left(u_{i}\left(z_{i}\right), u_{i}\left(w_{i}\right)\right),\left(\phi_{j}\left(u_{j}\left(b_{j}\right), u_{j}\left(w_{j}\right)\right)_{j \neq i}\right)\right. & \geq 0
\end{aligned}
$$

Due to skew symmetry of $\phi_{i}$ and oddness of $H$, (6.22) may be rewritten as:

$$
H\left(\phi_{i}\left(u_{i}\left(z_{i}\right), u_{i}\left(y_{i}\right)\right),\left(\phi_{j}\left(u_{j}\left(a_{j}\right), u_{j}\left(y_{j}\right)\right)_{j \neq i}\right) \leq 0\right.
$$

Using the increasingness of $H$ (resp. $\phi_{i}$ ) in its $i$ th (resp. first) argument, (6.21) and (6.24) imply $u_{i}\left(x_{i}\right) \geq u_{i}\left(z_{i}\right)$. Substituting $u_{i}\left(z_{i}\right)$ with $u_{i}\left(x_{i}\right)$ in equation (6.23) yields:

$$
H\left(\phi_{i}\left(u_{i}\left(x_{i}\right), u_{i}\left(w_{i}\right)\right),\left(\phi_{j}\left(u_{j}\left(b_{j}\right) u_{j}\left(w_{j}\right)\right)_{j \neq i}\right) \geq 0\right.
$$

which establishes $T A C 1_{i}$. The proof for $T A C 2_{i}$ is similar.

Sufficiency. From the proof of theorem 6.8 , we know that $\succsim$ has a representation in model ( $L 1 D 11)$ such that

$$
\begin{aligned}
\left(x_{i}, y_{i}\right) \succsim_{i}^{* *}\left(z_{i}, w_{i}\right) & \Leftrightarrow \phi_{i}\left(u_{i}\left(x_{i}\right), u_{i}\left(y_{i}\right)\right) \geq \phi_{i}\left(u_{i}\left(z_{i}\right), u_{i}\left(w_{i}\right)\right) \text { and } \\
x_{i} \succsim_{i}^{ \pm} y_{i} & \Leftrightarrow u_{i}\left(x_{i}\right) \geq u_{i}\left(y_{i}\right)
\end{aligned}
$$

In view of lemmas 5.15 and 6.12 , the proof will be complete if we show that after transforming the functions $\phi_{i}$ into functions $\varphi_{i}$ according to lemma 6.12 , it is still true that (5.18) holds, i.e. that:

$$
\begin{gathered}
\left.\begin{array}{c}
\left(z_{i}, w_{i}\right) \sim_{i}^{* *}\left(x_{i}, y_{i}\right) \text { and } \\
\exists a_{-i}, b_{-i} \in X_{-i} \text { s.t. }\left(x_{i}, a_{-i}\right) \sim\left(y_{i}, b_{-i}\right)
\end{array}\right\} \Rightarrow \\
\varphi_{i}\left(u_{i}\left(z_{i}\right), u_{i}\left(w_{i}\right)\right)=\varphi_{i}\left(u_{i}\left(x_{i}\right), u_{i}\left(y_{i}\right)\right) .
\end{gathered}
$$

In view of (6.18), this will be true if, as soon as $\left(z_{i}, w_{i}\right) \sim_{i}^{* *}\left(x_{i}, y_{i}\right)$ and $\exists a_{-i}, b_{-i} \in X_{-i}$ s.t. $\left(x_{i}, a_{-i}\right) \sim\left(y_{i}, b_{-i}\right)$, it is not true that $\phi_{i}\left(u_{i}\left(x_{i}\right), u_{i}\left(y_{i}\right)\right) \in$ $S$. This results from (4.2) and (4.3).

Remark 6.13 (Regular representations). For models (L1D8), (L1D7), (L1D9), (L1D10), (L1D7) and (L1D11), theorem 6.8 shows that it is always possible to build a representation of these models in which:

- $u_{i}$ is a numerical representation of the weak order $\succsim_{i}^{ \pm}$and 
- $\phi_{i}$ is a numerical representation of the weak order $\succsim_{i}^{*}$, in model (L1D8) and of the weak order $\succsim_{i}^{* *}$ in the more constrained models.

We call regular a representation in which this is the case (see Roberts (1979, ch. 2) about regularization of a scale of measurement).

In theorem 6.11, the representations that we build start from the regular representations provided by theorem 6.8 and we modify them, in accordance with the restrictions of lemma 5.15, so as to make $\phi_{i}$ increasing in its first argument and decreasing in its second argument. This modification is done, on each $i \in N$, on a set $S_{i}$ containing all the values $s$ such that:

$$
\begin{gathered}
s=\phi_{i}\left(u_{i}\left(x_{i}\right), u_{i}\left(z_{i}\right)\right)=\phi_{i}\left(u_{i}\left(y_{i}\right), u_{i}\left(z_{i}\right)\right), \text { or } \\
s=\phi_{i}\left(u_{i}\left(z_{i}\right), u_{i}\left(x_{i}\right)\right)=\phi_{i}\left(u_{i}\left(z_{i}\right), u_{i}\left(y_{i}\right)\right)
\end{gathered}
$$

for some $x_{i}, y_{i}, z_{i} \in X_{i}$ such that $x_{i} \succ_{i}^{ \pm} y_{i}$. It is not difficult to see that the emptiness of the sets $S_{i}$ is a necessary and sufficient condition to obtain a regular representation in the models envisaged in theorem 6.11 (see Bouyssou and Pirlot, 2002a, for details).

Remark 6.14 (Extension to the general case). Because we have deliberately used above representations that are not regular (i.e. $\phi_{i}$ does not necessarily represent $\succsim_{i}^{*}$ or $\succsim_{i}^{* *}$ ), the extension of the results in this section to the general uncountable case is slightly more involved than for other models (see Bouyssou and Pirlot, 2002a). This does not raise major difficulties however.

The main results in this section are summarized in tables 8 and 9 and figure 5 . 
Table 8

Models $(L 1 D 0)$ to $(L 1 D 11)$

\begin{tabular}{|c|c|c|}
\hline Models & Definition & Conditions \\
\hline$(L 1 D 0)$ & $\begin{array}{c}x \succsim y \Leftrightarrow H\left(\left[\phi_{i}\left(u_{i}\left(x_{i}\right), u_{i}\left(y_{i}\right)\right)\right]\right) \geq 0 \\
\phi_{i}(\nearrow, \searrow)\end{array}$ & $\varnothing$ \\
\hline$(L 1 D 1)$ & $(L 1 D 0)$ with $\phi_{i}\left(u\left(x_{i}\right), u_{i}\left(x_{i}\right)\right)=0$ & \\
\hline $\begin{array}{c}\Uparrow \\
(L 1 D 2)\end{array}$ & $(L 1 D 1)$ with $\phi_{i}$ skew symmetric & ind. \\
\hline$(L 1 D 3)$ & $(L 1 D 2)$ with $H$ odd & cpl., ind. \\
\hline$(L 1 D 4)$ & $\begin{array}{r}(L 1 D 0) \text { with } H(\nearrow)\end{array}$ & \\
\hline $\begin{array}{c}\mathbb{1} \\
(L 1 D 8)\end{array}$ & $(L 1 D 0)$ with $H(\nearrow \nearrow)$ & $R C 1, A C 123$ \\
\hline$(L 1 D 5)$ & $(L 1 D 1)$ with $H(\nearrow)$ & \\
\hline $\begin{array}{c}\mathbb{1} \\
(L 1 D 9)\end{array}$ & $(L 1 D 1)$ with $H(\nearrow \nearrow)$ & $R C 1$, ind., $A C 123$ \\
\hline$(L 1 D 6)$ & $(L 1 D 2)$ with $H(\nearrow)$ & \\
\hline $\begin{array}{c}\mathbb{1} \\
(L 1 D 10)\end{array}$ & $(L 1 D 2)$ with $H(\nearrow \nearrow)$ & $R C 12, A C 123$ \\
\hline$(L 1 D 7)$ & $(L 1 D 3)$ with $H(\nearrow)$ & cpl., $R C 12, A C 123$ \\
\hline$(L 1 D 11)$ & $(L 1 D 3)$ with $H(\nearrow / \nearrow)$ & cpl., $T C, A C 123$ \\
\hline
\end{tabular}

means increasing, $\nearrow$ means nondecreasing, \ means nonincreasing cpl. means completeness, ind. means independence

Conditions for the first four rows are identical to those of table 6 
Table 9

Models $(L 2 D 0)$ to $(L 2 D 11)$

\begin{tabular}{|c|c|c|}
\hline Models & Definition & Conditions \\
\hline$(L 2 D 0)$ & $\begin{array}{c}x \succsim y \Leftrightarrow H\left(\left[\phi_{i}\left(u_{i}\left(x_{i}\right), u_{i}\left(y_{i}\right)\right)\right]\right) \geq 0 \\
\phi_{i}(\nearrow \nearrow, \searrow \searrow)\end{array}$ & $\varnothing$ \\
\hline$(L 2 D 1)$ & $(L 1 D 0)$ with $\phi_{i}\left(u\left(x_{i}\right), u_{i}\left(x_{i}\right)\right)=0$ & \\
\hline $\begin{array}{c}\mathbb{1} \\
(L 2 D 2)\end{array}$ & $(L 2 D 1)$ with $\phi_{i}$ skew symmetric & ind. \\
\hline$(L 2 D 3)$ & $(L 2 D 2)$ with $H$ odd & cpl., ind. \\
\hline$(L 2 D 4)$ & $\begin{array}{l}(L 2 D 0) \text { with } H(\nearrow) \\
(I)\end{array}$ & \\
\hline $\begin{array}{c}\mathbb{1} \\
(L 2 D 8)\end{array}$ & $(L 2 D 0)$ with $H(\nearrow \nearrow)$ & $R C 1, A C 123$ \\
\hline$(L 2 D 5)$ & $(L 2 D 1)$ with $H(\nearrow)$ & \\
\hline $\begin{array}{c}\mathbb{1} \\
(L 2 D 9)\end{array}$ & $(L 2 D 1)$ with $H(\nearrow \nearrow)$ & $R C 1$, ind., $A C 123$ \\
\hline$\left(\begin{array}{c}L 2 D 6) \\
\Uparrow\end{array}\right.$ & $(L 2 D 2)$ with $H(\nearrow)$ & DC10 \\
\hline$(L 2 D 10)$ & $(L 2 D 2)$ with $H(\nearrow \nearrow)$ & 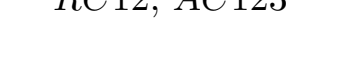 \\
\hline$(L 2 D 7)$ & $(L 2 D 3)$ with $H(\nearrow)$ & cpl., $R C 12, A C 123$ \\
\hline$(L 2 D 11)$ & $(L 2 D 3)$ with $H(\nearrow /)$ & cpl., TC, TAC 12 \\
\hline
\end{tabular}

Conditions are identical to those of table 8 except for the last row 
Figure 5. Implication between models resulting from theorems 6.8 and 6.11

$$
\begin{aligned}
& \text { (L2D11) } \\
& \Downarrow \\
& (L 1 D 11) \\
& (L 2 D 7) \Leftrightarrow(L 1 D 7) \\
& (L 2 D 10) \Leftrightarrow(L 1 D 10) \Leftrightarrow(L 2 D 6) \Leftrightarrow(L 1 D 6) \\
& (L 2 D 9) \Leftrightarrow(L 1 D 9) \Leftrightarrow(L 2 D 5) \Leftrightarrow(L 1 D 5) \\
& (L 2 D 8) \Leftrightarrow(L 1 D 8) \Leftrightarrow(L 2 D 4) \Leftrightarrow(L 1 D 4) \\
& (L 2 D 3) \Leftrightarrow(L 1 D 3) \Leftrightarrow(L 0 D 3) \vdots \\
& (L 2 D 2) \Leftrightarrow(L 1 D 2) \Leftrightarrow(L O D 2) \\
& (L 2 D 1) \Leftrightarrow(L 1 D 1) \Leftrightarrow(L 0 D 1) \\
& (L 2 D 0) \Leftrightarrow(L 1 D 0) \Leftrightarrow(L 0 D 0)
\end{aligned}
$$




\section{Discussion}

This paper has proposed a general approach of conjoint measurement models tolerating intransitivity and/or incompleteness using simple tools based on several kinds of marginal traces on coordinates induced by the binary relation on the product set. We have provided, when $X$ is supposed to be countable, a fairly complete analysis of a large variety of models. Our project was to investigate how far it was possible to go in terms of numerical representations using a limited number of cancellation conditions without imposing any transitivity requirement on the preference relation and any structural assumptions on the set of objects. Rather surprisingly, as we saw, such a poor framework allows us to go rather far. Furthermore the cancellation conditions that we used ( $R C 1, R C 2$, Independence, $T C, A C 1, A C 2, A C 3, T A C 1, T A C 2$ ) are reasonably simple and have close relations with the conditions used in the analysis of traditional conjoint measurement models.

Our framework and results have many possible applications. Among them let us mention:

- the characterization of all relations compatible with a dominance relation, using our models based on marginal traces on levels (see Bouyssou and Pirlot, 2002f),

- the characterization of preference relations that can be obtained using an "ordinal aggregation model" using our models based on marginal traces on levels (see Bouyssou and Pirlot, 2002b,c,e). Alternative approaches to this kind of models may be found in Bouyssou and Vansnick (1986); Dubois, Fargier, Perny, and Prad (2003b); Fargier and Perny (2001); Fishburn (1976); Tsoukiàs, Perny, and Vincke (2002)

- the characterization of various functional forms for F, G or $H$ (see Bouyssou, Greco, Matarazzo, P 2002; Greco, Matarazzo, and Słowiński, 2003),

- the particularization of our results to the important case of decision under uncertainty Bouyssou, Perny, and Pirlot (2000); Bouyssou and Pirlot (2003a),

- the characterization of "ordinal" models (see Dubois, Fargier, and Prade, 1997) for decision under uncertainty (see Bouyssou et al., 2000; Bouyssou and Pirlot, 2003b). Alternative approaches were proposed in Dubois, Fargier, and Perny (2003a); Dubois, Fargier, Prade, and Perny (2002).

It is clearly impossible to develop these points here. The patient reader who has followed us till now should be in position to guess the general spirit of these results.

Summarizing the main messages in a few words, we would say that:

- when confronted to a nontransitive/noncomplete relation, it is always prof- 
itable to investigate its traces and/or marginal traces,

- the use of conjoint measurement techniques is not restricted to the study of complete and transitive binary relations,

- if assessment procedures are not looked for, replacing additivity by mere decomposability requirements often allows to grasp in a very simple way the essence of a model,

- replacing additivity by mere decomposability requirements amounts to using models which are intimately related with "rule-based" preference modelling Greco, Matarazzo, and Słowiński (1999, 2001); Greco et al. (2002). This allows to consider the possibility of deriving assessment procedures using the machinery of "rule induction" in Artificial Intelligence.

Our framework and results are also intended to contribute to the general theory of conjoint measurement. They allow to draw the following general picture of conjoint measurement models (see figure 6), where models are classified, when studying the proposition $x \succsim y$, according to whether:

- they use traces on differences, i.e. their functional form can be written so as to be nondecreasing in functions $p_{i}\left(x_{i}, y_{i}\right)$,

- they use traces on levels, i.e. their functional form can be written so as to be nondecreasing in functions $u_{i}\left(x_{i}\right)$ and nonincreasing in functions $u_{i}\left(y_{i}\right)$,

- they are transitive.

These various models are summarized in figure 6 , where $\mathbf{T}$ denotes a transitive model, $\mathbf{L}$ a model involving traces on levels and $\mathbf{D}$ a model involving traces on differences. It is clear that the classical additive utility model (1.1) is transitive and involves traces on levels (via the functions $u_{i}$ ) and on differences (via the differences $\left.u_{i}\left(x_{i}\right)-u_{i}\left(y_{i}\right)\right)$.

In the $\mathbf{L}$ family all relations are weakly separable but may not be weakly independent (and, even less, independent). On the contrary, the D family includes only independent relations, as soon as axiom $R C 2$ is invoked. The marginal preference relations will be rather well-behaved in the $\mathbf{L}$ family being complete and most often semiorders (as soon as $A C 3$ and either $A C 1$ or $A C 2$ are invoked). This will not be the case in the $\mathbf{D}$ family.

It is worth noting that all combinations of $\mathbf{T}, \mathbf{L}$ and $\mathbf{D}$ have been studied in the literature except for the combination $\mathbf{T}, \overline{\mathbf{L}}, \mathbf{D}$. This is no surprise since when $\mathbf{D}$ applies, most models appeal to $R C 2$ and, hence, are independent. When this is combined with transitivity and completeness of $\succsim, \succsim_{i}$ is a weak order and is confounded with $\succsim_{i}^{ \pm}$. Hence, such models also involve traces on levels.

Our hope is that the proposed framework and results will stimulate research in the area. 
Figure 6. Summary of models

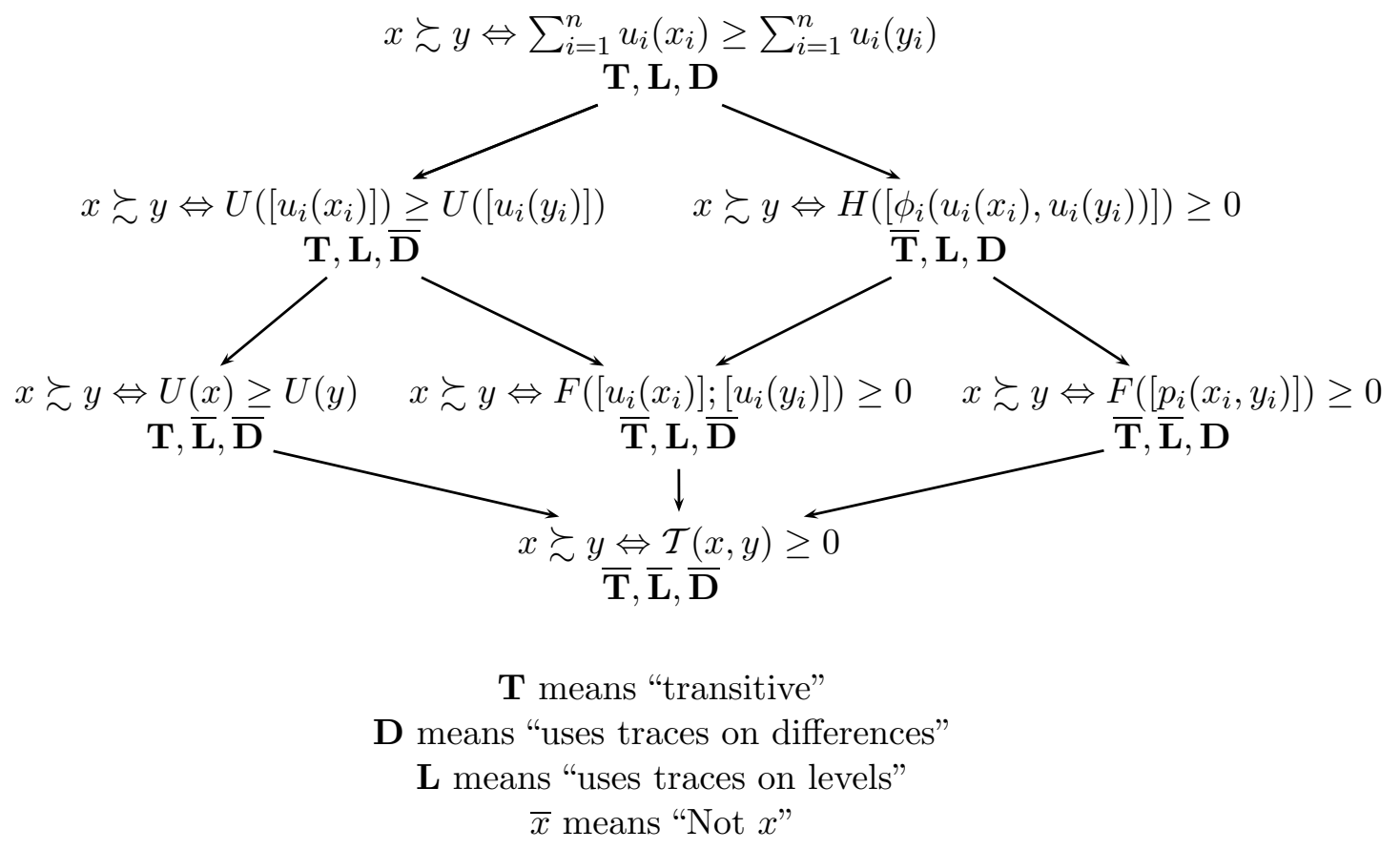

\section{A Examples}

\section{A.1 AC1, AC2, AC3, TAC1 and TAC2}

We first give 3 examples showing that, in the class of complete binary relations on $X, A C 1_{i}, A C 2_{i}$ and $A C 3_{i}$ are independent conditions. We leave to the reader the easy task of finding similar examples in the case of incomplete (e.g., irreflexive) binary relations. This will prove part 5 of lemma 4.2 . It is tedious, but easy, to check that completeness, $A C 1_{i}, A C 2_{i}$ and $A C 3_{i}$ are in fact completely independent conditions.

Examples A.1 to A.3 have a common structure. In all of them $X=X_{1} \times X_{2}$ with $X_{1}=\left\{w_{1}, x_{1}, y_{1}, z_{1}\right\}$ and $X_{2}=\left\{w_{2}, x_{2}, y_{2}, z_{2}\right\}$. We define $\succsim$ on $X$ by:

$$
\left(r_{1}, r_{2}\right) \succsim\left(s_{1}, s_{2}\right) \Leftrightarrow F\left[f\left(r_{1}, s_{1}\right) ; g\left(r_{2}, s_{2}\right)\right] \geq 0
$$

where $f$ (resp. $g$ ) is a real-valued function on $X_{1}^{2}$ (resp. on $X_{2}^{2}$ ) and $F$ is a real-valued function on $\mathbb{R}^{2}$.

It is clear that, using obvious notation:

(1) When $F$ is odd $(F(\mathbf{x})=-F(-\mathbf{x}))$ and $f$ and $g$ are skew symmetric $\left(f\left(r_{1}, s_{1}\right)=-f\left(s_{1}, r_{1}\right)\right), \succsim$ is complete. 
(2) When $F$ is nondecreasing in its first argument, $\left[f\left(r_{1}, t_{1}\right) \geq f\left(s_{1}, t_{1}\right)\right.$ for all $\left.t_{1} \in X_{1}\right] \Rightarrow r_{1} \succsim_{1}^{+} s_{1}$ and $\left[f\left(t_{1}, r_{1}\right) \leq f\left(t_{1}, s_{1}\right)\right.$ for all $\left.t_{1} \in X_{1}\right] \Rightarrow r_{1} \succsim_{1}^{-} s_{1}$. Similar conditions hold for the second component when $F$ is nonincreasing in its second argument.

In all the following examples, $f$ and $g$ will be skew symmetric and $F(\alpha, \beta)=$ $\alpha+\beta$, so that $F$ is odd and increasing in its two arguments.

Example A.1 ( $A C 1, A C 2$, Not $A C 3)$. Define $f$ and $g$ by the following tables (all tables are to be read from row to column):

\begin{tabular}{rrrrr}
$f$ & $w_{1}$ & $x_{1}$ & $y_{1}$ & $z_{1}$ \\
\hline$w_{1}$ & 0 & 0 & 0 & 0 \\
$x_{1}$ & 0 & 0 & 2 & 2 \\
$y_{1}$ & 0 & -2 & 0 & 1 \\
$z_{1}$ & 0 & -2 & -1 & 0
\end{tabular}

\begin{tabular}{rrrrr}
$g$ & $w_{2}$ & $x_{2}$ & $y_{2}$ & $z_{2}$ \\
\hline$w_{2}$ & 0 & 0 & 3 & 4 \\
$x_{2}$ & 0 & 0 & 3 & 4 \\
$y_{2}$ & -3 & -3 & 0 & 4 \\
$z_{2}$ & -4 & -4 & -4 & 0
\end{tabular}

Since the table for $g$ is step-typed, it is clear that $\succsim_{2}^{ \pm}$is complete. Hence, $A C 1_{2}, A C 2_{2}$ and $A C 3_{2}$ hold.

It is easily checked that $A C 1_{1}$ holds with $\succsim_{1}^{+}$as (using obvious simplified notation for weak orders): $\left[x_{1} ; w_{1}\right] \succ_{1}^{+} y_{1} \succ_{1}^{+} z_{1}$. Similarly, $A C 2_{1}$ holds with: $x_{1} \succ_{1}^{-} y_{1} \succ_{1}^{-}\left[w_{1} ; z_{1}\right]$.

Because $w_{1} \succ_{1}^{+} y_{1}$ and $y_{1} \succ_{1}^{-} w_{1}, A C 3_{1}$ is violated.

Hence we have an example of a complete binary relation satisfying $A C 1, A C 2$ and $A C 3_{i}$ on all but one attribute.

Example A.2 (Not $A C 1, A C 2, A C 3$ ). Define $f$ by the following table:

\begin{tabular}{rrrrr}
$f$ & $w_{1}$ & $x_{1}$ & $y_{1}$ & $z_{1}$ \\
\hline$w_{1}$ & 0 & 0 & 0 & 1 \\
$x_{1}$ & 0 & 0 & 2 & 3 \\
$y_{1}$ & 0 & -2 & 0 & 3 \\
$z_{1}$ & -1 & -3 & -3 & 0
\end{tabular}

and use the same table for $g$ as in example A.1. We have: $\operatorname{Not}\left[y_{1} \succsim_{1}^{+} w_{1}\right]$ (because $\left(w_{1}, w_{2}\right) \succsim\left(x_{1}, w_{2}\right)$ and $\left.\operatorname{Not}\left[\left(y_{1}, w_{2}\right) \succsim\left(x_{1}, w_{2}\right)\right]\right)$ and $\operatorname{Not}\left[w_{1} \succsim_{1}^{+}\right.$ $\left.y_{1}\right]$ (because $\left(y_{1}, y_{2}\right) \succsim\left(z_{1}, w_{2}\right)$ and $\left.\operatorname{Not}\left[\left(w_{1}, y_{2}\right) \succsim\left(z_{1}, w_{2}\right)\right]\right)$. Hence $A C 1_{1}$ is violated. In fact it is easy to check that $\succsim_{1}^{+}$is such that $x_{1} \succ_{1}^{+} y_{1}, x_{1} \succ_{1}^{+} w_{1}$, $x_{1} \succ_{1}^{+} z_{1}, y_{1} \succ_{1}^{+} z_{1}$ and $w_{1} \succ_{1}^{+} z_{1}$.

It is easily checked that $A C 2_{1}$ holds with $x_{1} \succ_{1}^{-}\left[y_{1} ; w_{1}\right] \succ_{1}^{-} z_{1}$. Using part 3 of lemma 4.2, we can check that $A C 3_{1}$ holds. 
Hence we have an example of a complete binary relation satisfying $A C 2, A C 3$ and $A C 1_{i}$ on all but one attribute.

Example A.3 ( $A C 1$, Not $A C 2, A C 3)$. Transposing the tables defining $f$ and $g$ in example A. 2 gives an example of a complete binary relation satisfying $A C 1, A C 3$ and $A C 2_{i}$ on all but one attribute.

The next two examples are related to lemma 4.5. We first show that there are weakly independent semiorders satisfying $T A C 12$ that are not weak orders.

Example A.4 (Nontransitive semiorder satisfying TAC12). Let $X=$ $X_{1} \times X_{2}$ with $X_{1}=\left\{x_{1}, y_{1}, z_{1}\right\}$ and $X_{2}=\left\{x_{2}, y_{2}, z_{2}\right\}$. Consider the binary relation $\succsim$ identical to the weak order: $\left(x_{1}, x_{2}\right)>\left(x_{1}, y_{2}\right)>\left(y_{1}, x_{2}\right)>\left(x_{1}, z_{2}\right)>$ $\left(y_{1}, y_{2}\right)>\left(z_{1}, x_{2}\right)>\left(y_{1}, z_{2}\right)>\left(z_{1}, y_{2}\right)>\left(z_{1}, z_{2}\right)$, except that $\left(y_{1}, y_{2}\right) \sim\left(x_{1}, z_{2}\right)$ and $\left(z_{1}, x_{2}\right) \sim\left(y_{1}, y_{2}\right)$

This relation is clearly complete. It is not transitive since $\left(z_{1}, x_{2}\right) \succsim\left(y_{1}, y_{2}\right)$, $\left(y_{1}, y_{2}\right) \succsim\left(x_{1}, z_{2}\right)$ but $\left(x_{1}, z_{2}\right) \succ\left(z_{1}, x_{2}\right)$.

It is easily checked that this relation is a semiorder having the preceding weak order for trace. This semiorder is independent. Its marginal relations are weak orders identical to its marginal traces. We have $x_{1}>y_{1}>z_{1}$ and $x_{2}>y_{2}>z_{2}$.

This relation has only a few pairs of alternatives linked by $\sim$. It is then easy to check that TAC12 holds. For instance, starting with $\left(y_{1}, y_{2}\right) \succsim\left(x_{1}, z_{2}\right)$ we should have $\left(x_{1}, y_{2}\right) \succ\left(x_{1}, z_{2}\right),\left(y_{1}, x_{2}\right) \succ\left(x_{1}, z_{2}\right)$ and $\left(y_{1}, y_{2}\right) \succ\left(y_{1}, z_{2}\right)$, because $x_{1} \succ_{1}^{ \pm} y_{1}$ and $x_{2} \succ_{2}^{ \pm} y_{2}$. This is indeed the case.

Hence we have an example of a nontransitive weakly independent semiorder satisfying $T A C 12$.

The final example shows that for complete relations, TAC2 may hold without $T A C 1$. An example of complete relation verifying TAC1 but not TAC2 is easily built using a similar principle.

Example A.5 (Not $T A C 1, T A C 2$ ). Let $X=X_{1} \times X_{2}$ with $X_{1}=\mathbb{R}^{2}$ and $X_{2}=\mathbb{R}$.

Define $\succsim$ letting:

$$
\left(\left(a_{1}, b_{1}\right), x_{2}\right) \succsim\left(\left(c_{1}, d_{1}\right), y_{2}\right) \Leftrightarrow a_{1}+x_{2}>c_{1}+y_{2} \text { or }\left\{\begin{array}{l}
a_{1}+x_{2}=c_{1}+y_{2} \\
\text { and } \\
a_{1}+b_{1} \geq c_{1} .
\end{array}\right.
$$

It is clear that $\succsim$ is complete.

We have $\left(a_{1}, b_{1}\right) \succsim_{1}^{-}\left(c_{1}, d_{1}\right) \Leftrightarrow a_{1} \geq c_{1}$ and $\left(a_{1}, b_{1}\right) \succsim_{1}^{+}\left(c_{1}, d_{1}\right) \Leftrightarrow\left(a_{1}, b_{1}\right) \geq_{L}$ 
$\left(c_{1}, d_{1}\right)$, where $\geq_{L}$ denotes the lexicographic order on $\mathbb{R}^{2}$. On the second attribute, it is clear that $x_{2} \succsim_{2}^{+} y_{2} \Leftrightarrow x_{2} \succsim_{2}^{-} y_{2} \Leftrightarrow x_{2}>y_{2}$.

A simple check shows that $\succsim$ is strictly responsive to $\succsim_{2}^{+}, \succsim_{2}^{-}$and $\succsim_{1}^{-}$. This not so for $\succsim_{1}^{+}$since, for instance, $((10,0), 10) \sim((8,2), 12)$ and $((10,2), 10) \sim$ $((8,2), 12)$, while $(10,2) \succ_{1}^{+}(10,0)$ (because $((10,2), 10) \succsim((11,0), 9)$ and $\operatorname{Not}[((10,0), 10) \succsim((11,0), 9)])$.

Hence we have an example of a complete relation satisfying $T A C 2$ and $T A C 1_{2}$ but violating $T A C 1_{1}$.

\section{A.2 $R C 1$ and $R C 2$}

We first give six examples complete the proof of part 3 of lemma 5.3 showing that independence, reflexivity and $R C 1_{i}$ are completely independent conditions.

Example A.6 (reflexive, $R C 1$, not independent). Let $X=\{a, b\} \times\{z, w\}$ and consider $\succsim$ on $X$ defined by: for all $(\alpha, \beta),(\gamma, \delta) \in X,(\alpha, \beta) \succsim(\gamma, \delta) \Leftrightarrow$ $f(\alpha, \gamma)+g(\beta, \delta) \geq 0$, where $f$ and $g$ are such that: $f(a, a)=-1, f(a, b)=0.5$, $f(b, a)=-0.5, f(b, b)=1, g(z, z)=g(w, w)=g(w, z)=1, g(z, w)=0$

It is easy to see that $\succsim$ is reflexive and satisfies $R C 1$. It is not independent since $(b, z) \succsim(b, w)$ and $\operatorname{Not}[(a, z) \succsim(a, w)]$.

Example A.7 ( $R C 1$, not reflexive, not independent). In example A.6, taking $f(a, a)=-2$ leads to a relation $\succsim$ that verifies $R C 1$ but is neither independent nor reflexive (since $\operatorname{Not}[(a, z) \succsim(a, z)])$.

Example A.8 ( $R C 1$, not reflexive, independent). Let $X=\{a, b\} \times\{z, w\}$ and consider $\succsim$ on $X$ defined by: for all $(\alpha, \beta),(\gamma, \delta) \in X,(\alpha, \beta) \succsim(\gamma, \delta) \Leftrightarrow$ $f(\alpha, \gamma)+g(\beta, \delta) \geq 0$, where $f$ and $g$ are such that: $f(a, a)=f(b, b)=f(b, a)=$ $-1, f(a, b)=1, g(z, z)=g(w, w)=0, g(z, w)=1, g(w, z)=-1$

It is easy to see that $\succsim$ is not reflexive (it is in fact irreflexive). It is easily seen to satisfy $R C 1$. Since $f(a, a)=f(b, b)$ and $g(z, z)=g(w, w), \succsim$ is clearly independent.

Example A.9 (not $R C 1$, reflexive, independent). Let $X=\{a, b, c\} \times$ $\{z, w\}$ and consider $\succsim$ on $X$ that is a clique (with all loops) except that $\operatorname{Not}[(a, z) \succsim(c, w)]$ and $\operatorname{Not}[(a, w) \succsim(b, z)]$.

It is clear that $\succsim$ is reflexive. It can easily be checked that $\succsim$ is independent. It does not satisfy $R C 1$ since: $(a, z) \succsim(b, w),(a, w) \succsim(c, z), \operatorname{Not}[(a, z) \succsim(c, w)]$ 
and $\operatorname{Not}[(a, w) \succsim(b, z)]$.

Example A.10 (not $R C 1$, not reflexive, independent). Modifying example A.9 in order to have $\succsim$ irreflexive gives an example of a relation that is independent but violates $R C 1$ and reflexivity.

Example A.11 (not $R C 1$, reflexive, not independent). Modifying example A.9 in order to have $\operatorname{Not}[(b, z) \succsim(b, w)]$ leads to a relation $\succsim$ that is reflexive but violates independence and $R C 1$.

We now give three examples that complete the proof of part 4 of lemma 5.3 showing that $R C 1_{i}$ and $R C 2_{i}$ are completely independent conditions in the class of complete relations.

Example A.12 (not $R C 1$, not $R C 2$ ). Let $X=\{a, b, c\} \times\{z, w, k\}$ and consider $\succsim$ on $X$ that is a clique (with all loops) except that $\operatorname{Not}[(a, z) \succsim$ $(c, w)], \operatorname{Not}[(a, k) \succsim(b, z)]$ and $\operatorname{Not}[(c, z) \succsim(a, w)]$. It is clear that $\succsim$ is complete. Since $(a, z) \succsim(b, w),(c, k) \succsim(a, z), \operatorname{Not}[(a, k) \succsim(b, z)]$ and $\operatorname{Not}[(c, z) \succsim(a, w)], \succsim$ violates $R C 1$. Since $(a, z) \succsim(b, w),(b, z) \succsim(a, w)$, $\operatorname{Not}[(a, z) \succsim(c, w)]$ and $\operatorname{Not}[(c, z) \succsim(a, w)], \succsim$ violates $R C 2$.

Example A.13 (not $R C 1, R C 2$ ). Modify example A.12 adding the relation $(a, z) \succsim(c, w)$. It is clear that $\succsim$ is complete and violates $R C 1$. Using lemma 5.2 , it is not difficult to see that it satisfies $R C 2$.

Example A.14 ( $R C 1$, not $R C 2)$. Let $X=\{a, b\} \times\{z, w\}$ and consider $\succsim$ on $X$ defined by: for all $(\alpha, \beta),(\gamma, \delta) \in X,(\alpha, \beta) \succsim(\gamma, \delta) \Leftrightarrow f(\alpha, \gamma)+$ $g(\beta, \delta) \geq 0$, where $f$ and $g$ are such that: $f(a, a)=-1, f(a, b)=f(b, a)=$ $f(b, b)=1, g(z, w)=0, g(z, z)=g(w, w)=g(w, z)=1$.

It is easy to see that $\succsim$ is complete and satisfies $R C 1$. It is not independent since $(b, z) \succsim(b, w)$ and $\operatorname{Not}[(a, z) \succsim(a, w)]$. In view of part 2 of lemma 5.3, this shows that $R C 2$ is violated.

\section{A.3 TC, $A C 1, A C 2, A C 3$}

We show below that, in the class of complete binary relations conditions $T C_{i}$, $A C 1_{i}, A C 2_{i}$ and $A C 3_{i}$ are independent.

Example A.15 (AC1,AC2, $A C 3$, Not $T C)$. Let $X=\{a, b, c\} \times\{d, e, f\}$; $x \succsim y$ iff $G\left(p_{1}\left(x_{1}, y_{1}\right), p_{2}\left(x_{2}, y_{2}\right) \geq 0\right.$ with

$$
G(\alpha, \beta)=\left\{\begin{array}{cl}
\alpha+\beta & \text { if }|\alpha+\beta|>2 \\
0 & \text { otherwise }
\end{array}\right.
$$


and $p_{1}$ and $p_{2}$ given in the following tables:

\begin{tabular}{r|rrr}
$p_{1}$ & $a$ & $b$ & $c$ \\
\hline$a$ & 0 & -2 & -1 \\
$b$ & 2 & 0 & 1 \\
$c$ & 1 & -1 & 0
\end{tabular}

\begin{tabular}{r|rrr}
$p_{2}$ & $d$ & $e$ & $f$ \\
\hline$d$ & 0 & 0 & -2 \\
$e$ & 0 & 0 & -2 \\
$f$ & 2 & 2 & 0
\end{tabular}

$G$ is odd and nondecreasing and $p_{1}, p_{2}$ are skew symmetric; hence $\succsim$ is complete and satisfies $R C 1, R C 2$. Condition $T C$ is violated since $(c, d) \succsim(a, f),(a, e) \succsim$ $(c, d),(a, d) \succsim(b, e)$ but $N o t[(a, d) \succsim(b, f)]$.

It is easily checked that $A C 1, A C 2$ and $A C 3$ hold with $b \succ_{1}^{ \pm} c \succ_{1}^{ \pm} a$ and $f \succ_{2}^{ \pm}\left[d \sim_{2}^{ \pm} e\right]$.

Example A.16 (Not $A C 1, A C 2, A C 3, T C$ ). Let $X=\{a, b, c\} \times\{d, e, f\}$. Define $\succsim$ letting $x \succsim y$ iff $G\left(p_{1}\left(x_{1}, y_{1}\right), p_{2}\left(x_{2}, y_{2}\right) \geq 0\right.$, with $p_{1}$ and $p_{2}$ given in the following tables:

\begin{tabular}{c|rrr}
$p_{1}$ & $a$ & $b$ & $c$ \\
\hline$a$ & 0 & 2 & -1 \\
$b$ & -2 & 0 & -1 \\
$c$ & 1 & 1 & 0
\end{tabular}

\begin{tabular}{c|rrr}
$p_{2}$ & $d$ & $e$ & $f$ \\
\hline$d$ & 0 & 2 & 2 \\
$e$ & -2 & 0 & 2 \\
$f$ & -2 & -2 & 0
\end{tabular}

and $G$ such that:

\begin{tabular}{r|rrrrr}
$G$ & -2 & -1 & 0 & 1 & 2 \\
\hline-2 & -41 & -30 & -21 & -10 & 0 \\
-1 & -31 & -20 & -9 & 0 & 10 \\
0 & -19 & -11 & 0 & 11 & 19 \\
1 & -10 & 0 & 9 & 20 & 31 \\
2 & 0 & 10 & 21 & 30 & 41
\end{tabular}

$G$ is odd and increasing in its two arguments and $p_{1}, p_{2}$ are skew symmetric implying that $\succsim$ is complete and satisfies $T C$.

It is easy to check that we have: $c \succ_{1}^{-} a \succ_{1}^{-} b, c \succ_{1}^{+} b, a \succ_{1}^{+} b, \operatorname{Not}\left[c \succsim_{1}^{ \pm} a\right]$, $N o t\left[a \succsim_{1}^{ \pm} c\right], d \succ_{2}^{ \pm} e \succ_{2}^{ \pm} f$.

Hence $A C 2$ and $A C 3$ hold but $A C 1_{1}$ is violated (while $A C 1_{2}$ holds). One verifies indeed that we have $(c, f) \succsim(c, f)$ and $(a, f) \succsim(b, e)$ but neither $(a, f) \succsim(c, f)$ nor $(c, f) \succsim(b, e)$.

Example A.17 ( $A C 1$, Not $A C 2, A C 3, T C)$. This example is the same as example A.16 except that $p_{1}$ becomes $-p_{1}$. The effect of this modification is to interchange the rôles of $A C 1_{1}$ and $A C 2_{1}$ since the value associated to the pair $\left(y_{1}, x_{1}\right)$ is the value that was formerly associated to $\left(x_{1}, y_{1}\right)$ in example A.16. Therefore $\succsim$ is complete and verifies $T C$. 
We have: $b \succ_{1}^{+} a \succ_{1}^{+} c, b \succ_{1}^{-} c, b \succ_{1}^{-} a, \operatorname{Not}\left[a \succsim_{1}^{-} c\right], N o t\left[c \succsim_{1}^{-} a\right], d \succ_{2}^{ \pm} e \succ_{2}^{ \pm}$ $f$. Hence $A C 1$ and $A C 3$ hold but $A C 2_{1}$ is violated (while $A C 2_{2}$ holds). One verifies indeed that $(c, f) \succsim(c, f)$ and $(b, e) \succsim(a, d)$ but neither $(c, f) \succsim(a, f)$ nor $(b, e) \succsim(c, d)$.

Example A.18 $(A C 1, A C 2$, Not $A C 3, T C)$. Let $X=\{a, b, c, d\} \times\{w, x, y, z\}$. Define $\succsim$ as in example A.16 with the same table for $G$ and $p_{1}, p_{2}$ given in the following tables:

\begin{tabular}{c|rrrr}
$p_{1}$ & $a$ & $b$ & $c$ & $d$ \\
\hline$a$ & 0 & 1 & 2 & 2 \\
$b$ & -1 & 0 & 1 & 0 \\
$c$ & -2 & -1 & 0 & -2 \\
$d$ & -2 & 0 & 2 & 0
\end{tabular}

\begin{tabular}{r|rrrr}
$p_{2}$ & $w$ & $x$ & $y$ & $z$ \\
\hline$w$ & 0 & 2 & 2 & 2 \\
$x$ & -2 & 0 & 2 & 2 \\
$y$ & -2 & -2 & 0 & 2 \\
$z$ & -2 & -2 & -2 & 0
\end{tabular}

Since $G$ is odd and increasing and $p_{1}, p_{2}$ are skew symmetric, we know that $\succsim$ is complete and verifies $T C$.

It can be checked that we have: $w \succ_{2}^{ \pm} x \succ_{2}^{ \pm} y \succ_{2}^{ \pm} z, a \succ_{1}^{+} d \succ_{1}^{+} b \succ_{1}^{+} c$ and $a \succ_{1}^{-} b \succ_{1}^{-} d \succ_{1}^{-} c$. Hence $A C 1$ and $A C 2$ hold but $A C 3$ is violated since neither $d \succsim_{1}^{ \pm} b$ nor $b \succsim_{1}^{ \pm} d$.

\section{B Proof of lemma 6.12}

We prove lemma 6.12 in the most constrained case, i.e. when $f$ is skew symmetric. Since $U$ is countable, the set $S$ of values $r$ for which there are $a, b, c, d \in U$ such that either:

$$
\begin{aligned}
f(a, c)=f(b, c)=r & \text { with } a>b \text { or } \\
f(a, c)=f(a, d)=r & \text { with } c>d,
\end{aligned}
$$

is countable. Consider separately the positive part $S^{+}(r>0)$, the null part $S^{=}(r=0)$ and the negative part $S^{-}(r<0)$ of $S$. We number arbitrarily the elements of $S^{+}$and $S^{-}$

$$
\begin{aligned}
& r_{1}^{+}, r_{2}^{+}, \ldots \text { for the elements of } S^{+} \\
& r_{1}^{-}, r_{2}^{-}, \ldots \text { for the elements of } S^{-} .
\end{aligned}
$$


For each $u, v$ in $U \times U$, we define $g(u, v)$ letting:

$$
\begin{gathered}
\qquad(u, v)= \\
\begin{cases}u-v & \text { if } f(u, v)=0, \\
f(u, v)+1+\sum_{k: r_{k}^{+}<f(u, v)}(1 / 2)^{k} & \text { if } f(u, v)>0 \text { and } f(u, v) \notin S \\
r_{i}^{+}+1+\sum_{k: r_{k}^{+}<r_{i}^{+}}(1 / 2)^{k}+(1 / 2)^{i+1}(1+u-v) & \text { if } f(u, v)=r_{i}^{+} \\
f(u, v)-1-\sum_{k: r_{k}^{-}>f(u, v)}(1 / 2)^{k} & \text { if } f(u, v)<0 \text { and } f(u, v) \notin S \\
r_{i}^{-}-1-\sum_{k: r_{k}^{-}>r_{i}^{-}}(1 / 2)^{k}-(1 / 2)^{i+1}(1-u+v) & \text { if } f(u, v)=r_{i}^{-}\end{cases}
\end{gathered}
$$

The function $g$ is now fully described. It is skew symmetric since $f$ is. It is strictly monotonic on $f^{-1}(r)$, for all $r \in S$. By construction, we have:

$$
\left.\left[f(u, v)=f\left(u^{\prime}, v^{\prime}\right)\right] \Rightarrow[g(u, v))=g\left(u^{\prime}, v^{\prime}\right)\right] \text { iff } f(u, v) \notin S
$$

It is easy to check that $g$ is increasing in its first argument, decreasing in its second argument and such that (6.17) holds.

\section{References}

Aleskerov, F., Monjardet, B., 2002. Utility Maximization, Choice and Preference. Springer Verlag, Heidelberg.

Anscombe, F. J., Aumann, R. J., 1963. A definition of subjective probability. Annals of Mathematical Statistics 34, 199-205.

Atkinson, A. B., 1970. On the measurement of inequality. Journal of Economic Theory 2, 244-263.

Belton, V., Stewart, T., 2001. Multiple Criteria Decision Analysis: An Integrated Approach. Kluwer, Dordrecht.

Ben-Porath, E., Gilboa, I., 1994. Linear measures, the Gini index and the income-equality tradeoff. Journal of Economic Theory 64, 443-467.

Ben-Porath, E., Gilboa, I., Schmeidler, D., 1997. On the measurement of inequality under uncertainty. Journal of Economic Theory 75, 194-204.

Blackorby, C., Primont, D., Russell, R., 1978. Duality, separability, and functional structure: Theory and economic applications. North-Holland, New York.

Bouyssou, D., 1986. Some remarks on the notion of compensation in MCDM. European Journal of Operational Research 26, 150-160.

Bouyssou, D., 1990. Building criteria: A prerequisite for MCDA. In: Bana e Costa, C. A. (Ed.), Readings in multiple criteria decision aid. SpringerVerlag, pp. 58-80.

Bouyssou, D., Greco, S., Matarazzo, B., Pirlot, M., Słowiński, R., July 2002. Characterization of 'max', 'min' and 'order statistics' multicriteria aggregation functions, communication to IFORS'2002, 8-12 July, 2002, Edinburgh, U.K. 
Bouyssou, D., Perny, P., Pirlot, M., 2000. Nontransitive decomposable conjoint measurement as a general framework for MCDM and decision under uncertainty, communication to EURO XVII, Budapest, Hungary, 16-19 July.

Bouyssou, D., Pirlot, M., 1999. Conjoint measurement without additivity and transitivity. In: Meskens, N., Roubens, M. (Eds.), Advances in Decision Analysis. Kluwer, Dordrecht, pp. 13-29.

Bouyssou, D., Pirlot, M., 2002a. 'Additive difference' models without additivity and subtractivity, working Paper, LAMSADE, Université Paris Dauphine, submitted, available at www. lamsade. dauphine.fr/ bouyssou/.

Bouyssou, D., Pirlot, M., 2002b. A characterization of concordance relations, working Paper, LAMSADE, Université Paris-Dauphine, submitted, available at www. lamsade.dauphine.fr/ ־bouyssou/.

Bouyssou, D., Pirlot, M., 2002c. A characterization of strict concordance relations. In: Bouyssou, D., Jacquet-Lagrèze, E., Perny, P., Słowiński, R., Vanderpooten, D., Vincke, Ph. (Eds.), Aiding Decisions with Multiple Criteria: Essays in Honour of Bernard Roy. Kluwer, Dordrecht, pp. 121-145.

Bouyssou, D., Pirlot, M., 2002d. Nontransitive decomposable conjoint measurement. Journal of Mathematical Psychology 46 (6), 677-703.

Bouyssou, D., Pirlot, M., 2002e. Ordinal aggregation and strict preferences for multiattributed alternatives, working Paper, LAMSADE, Université Paris-Dauphine, submitted, available at www. lamsade. dauphine.fr/ bouyssou/.

Bouyssou, D., Pirlot, M., 2002f. Preferences for multiattributed alternatives: Traces, dominance, and numerical representations, working Paper, LAMSADE, Université Paris Dauphine, submitted, available at www. lamsade.dauphine.fr/ ${ }^{\text {bouyssou/. }}$

Bouyssou, D., Pirlot, M., 2003a. A note on Wakker's cardinal coordinate independence, working Paper, LAMSADE, Université Paris-Dauphine, submitted, available at www. Iamsade.dauphine.fr/ bouyssou/.

Bouyssou, D., Pirlot, M., 2003b. On some ordinal models for decision making under uncertainty, working Paper, LAMSADE, Université Paris-Dauphine.

Bouyssou, D., Pirlot, M., Vincke, Ph., 1997. A general model of preference aggregation. In: Karwan, M. H., Spronk, J., Wallenius, J. (Eds.), Essays in Decision Making. Springer Verlag, Berlin, pp. 120-134.

Bouyssou, D., Vansnick, J.-C., 1986. Noncompensatory and generalized noncompensatory preference structures. Theory and Decision 21, 251-266.

Dahlstrand, V., Montgomery, H., 1984. Information search and evaluation processes in decision-making: A computer-based process tracking study. Acta Psychologica 56, 113-123.

Debreu, G., 1959. Theory of value: An axiomatic analysis of economic equilibrium. Wiley, New York.

Debreu, G., 1960. Topological methods in cardinal utility theory. In: Arrow, K. J., Karlin, S., Suppes, P. (Eds.), Mathematical methods in the Social Sciences. Stanford University Press, Stanford, pp. 16-26. 
Doignon, J.-P., Ducamp, A., Falmagne, J.-C., 1984. On realizable biorders and the biorder dimension of a relation. Journal of Mathematical Psychology 28, 73-109.

Doignon, J.-P., Monjardet, B., Roubens, M., Vincke, Ph., 1988. Biorder families, valued relations and preference modelling. Journal of Mathematical Psychology 30, 435-480.

Dubois, D., Fargier, H., Perny, P., 2003a. Qualitative decision theory with preference relations and comparative uncertainty: An axiomatic approach. Artificial Intelligence 148, 219-260.

Dubois, D., Fargier, H., Perny, P., Prade, H., 2003b. A characterization of generalized concordance rules in multicriteria decision-making. International Journal of Intelligent Systems 18 (7), 751-774.

Dubois, D., Fargier, H., Prade, H., 1997. Decision-making under ordinal preferences and uncertainty. In: Geiger, D., Shenoy, P. P. (Eds.), Proceedings of the 13th conference on uncertainty in artificial intelligence. Morgan Kaufmann, Los Altos, pp. 157-164.

Dubois, D., Fargier, H., Prade, H., Perny, P., 2002. Qualitative decision theory: from Savage's axioms to nonmonotonic reasoning. Journal of the ACM 49 (4), 455-495.

Ducamp, A., Falmagne, J.-C., 1969. Composite measurement. Journal of Mathematical Psychology 6, 359-390.

Ebert, U., 1985. Non-transitive representations of transitive orderings. Economic Letters 18, 109-112.

Färe, R., Primont, D., 1981. Separability vs. weak separability: A further result. Journal of Economic Theory 25, 455-460.

Fargier, H., Perny, P., 2001. Modélisation des préférences par une règle de concordance généralisée. In: Colorni, A., Paruccini, M., Roy, B. (Eds.), AMCD-A, Aide Mulcritère à la Décision/Multiple Criteria Decision Aid. European Commission, Joint Research Centre, pp. 99-115.

Fishburn, P. C., 1970a. Intransitive indifference in preference theory: A survey. Operations Research 18 (2), 207-228.

Fishburn, P. C., 1970b. Utility theory for decision-making. Wiley, New York.

Fishburn, P. C., 1973. Interval representations for interval orders and semiorders. Journal of Mathematical Psychology 10, 91-105.

Fishburn, P. C., 1976. Noncompensatory preferences. Synthese 33, 393-403.

Fishburn, P. C., 1985. Interval orders and intervals graphs. Wiley, New York.

Fishburn, P. C., 1988. Normative theories of decision making under risk and under uncertainty. In: Kacprzyk, J., Roubens, M. (Eds.), Nonconventional preference relations in decision making. Springer, Berlin, pp. 1-21.

Fishburn, P. C., 1990a. Additive non-transitive preferences. Economic Letters 34, 317-321.

Fishburn, P. C., 1990b. Continuous nontransitive additive conjoint measurement. Mathematical Social Sciences 20, 165-193.

Fishburn, P. C., 1991a. Nontransitive additive conjoint measurement. Journal of Mathematical Psychology 35, 1-40. 
Fishburn, P. C., 1991b. Nontransitive preferences in decision theory. Journal of Risk and Uncertainty 4, 113-134.

Fishburn, P. C., 1992a. Additive differences and simple preference comparisons. Journal of Mathematical Psychology 36, 21-31.

Fishburn, P. C., 1992b. A general axiomatization of additive measurement with applications. Naval Research Logistics 39 (6), 741-755.

Fishburn, P. C., 1992c. On nonstandard nontransitive additive utility. Journal of Economic Theory 56, 426-433.

French, S., 1993. Decision theory - An introduction to the mathematics of rationality. Ellis Horwood, London.

Furkhen, G., Richter, M. K., 1991. Additive utility. Economic Theory 1, 83105.

Gilboa, I., Lapson, R., 1995. Aggregation of semiorders: Intransitive indifference makes a difference. Economic Theory 5, 109-126.

Goldstein, W. M., 1991. Decomposable threshold models. Journal of Mathematical Psychology 35, 64-79.

Gonzales, Ch., 1996. Additive utilities when some components are solvable and others not. Journal of Mathematical Psychology 40, 141-151.

Gonzales, Ch., 2000. Two factor additive conjoint measurement with one solvable component. Journal of Mathematical Psychology 44, 285-309.

Gonzales, Ch., 2003. Additive utility without restricted solvavibility on every component. Journal of Mathematical Psychology 47, 47-65.

Greco, S., Matarazzo, B., Słowiński, R., 1999. The use of rough sets and fuzzy sets in MCDM. In: Gal, T., Hanne, T., Stewart, T. (Eds.), Multicriteria decision making, Advances in MCDM models, algorithms, theory and applications. Kluwer, Dordrecht, pp. 14.1-14.59.

Greco, S., Matarazzo, B., Słowiński, R., 2001. Rough sets theory for multicriteria decision analysis. European Journal of Operational Research 129 (1), $1-47$.

Greco, S., Matarazzo, B., Słowiński, R., 2002. Preference representation by means of conjoint measurement and decision rule model. In: Bouyssou, D., Jacquet-Lagrèze, E., Perny, P., Słowiński, R., Vanderpooten, D., Vincke, Ph. (Eds.), Aiding Decisions with Multiple Criteria: Essays in Honour of Bernard Roy. Kluwer, Dordrecht, pp. 263-313.

Greco, S., Matarazzo, B., Słowiński, R., 2003. Axiomatic characterization of a general utility function and its particular cases in terms of conjoint measurement and rough-set decision rules. forthcoming in European Journal of Operational Research.

Gul, F., 1992. Savage's theorem with a finite number of states. Journal of Economic Theory 57, 99-110.

Jaffray, J.-Y., 1974. On the extension of additive utilities to infinite sets. Journal of Mathematical Psychology 11, 431-452.

Karni, E., Safra, Z., 1998. The hexagon condition and additive representation for two dimensions: An algebraic approach. Journal of Mathematical Psychology 42, 393-399. 
Keeney, R. L., Raiffa, H., 1976. Decisions with multiple objectives: Preferences and value tradeoffs. Wiley, New York.

Köbberling, V., 2003. Comment on: Edi Karni \& Zvi Safra (1998) The hexagon condition and additive representation for two dimensions: An algebraic approach. Journal of Mathematical Psychology 47 (3), 370.

Koopmans, T. C., 1960. Stationary ordinal utility and impatience. Econometrica 28, 287-309.

Koopmans, T. C., 1972. Representation of preference orderings over time. In: McGuire, C. B., Radner, R. (Eds.), Decision and Organization. NorthHolland, Amsterdam, pp. 57-100.

Krantz, D. H., Luce, R. D., Suppes, P., Tversky, A., 1971. Foundations of measurement, vol. 1: Additive and polynomial representations. Academic Press, New York.

Laslier, J.-F., 1997. Tournament solutions and majority voting. Springer Verlag, Berlin.

Luce, R. D., 1956. Semiorders and a theory of utility discrimination. Econometrica 24, 178-191.

Mak, K.-T., 1984. Notes on separable preferences. Journal of Economic Theory 33, 309-321.

May, K. O., 1954. Intransitivity, utility and the aggregation of preference patterns. Econometrica 22, 1-13.

Monjardet, B., 1978. Axiomatiques et propriétés des quasi-ordres. Mathématiques et Sciences Humaines 63, 51-82.

Montgomery, H., 1977. A study of intransitive preferences using a think aloud procedure. In: Jungerman, H., de Zeeuw, G. (Eds.), Decision-making and Change in Human Affairs. D. Reidel, Dordrecht, pp. 347-362.

Montgomery, H., Svenson, O., 1976. On decision rules and information processing strategies for choice among multiattribute alternatives. Scandinavian Journal of Psychology 17, 283-291.

Nakamura, Y., 2002. Additive utility on densely ordered sets. Journal of Mathematical Psychology 46, 515-530.

Pirlot, M., Vincke, Ph., 1997. Semiorders. Properties, representations, applications. Kluwer, Dordrecht.

Roberts, F. S., 1979. Measurement theory with applications to decision making, utility and the social sciences. Addison-Wesley, Reading.

Roubens, M., Vincke, Ph., 1985. Preference modelling. Springer Verlag, Berlin.

Roy, B., 1996. Multicriteria methodology for decision aiding. Kluwer, Dordrecht, original version in French, Méthodologie multicritère d'aide à la décision, Economica, Paris, 1985.

Roy, B., Bouyssou, D., 1993. Aide multicritère à la décision : Méthodes et cas. Economica, Paris.

Russo, J. E., Dosher, B. A., 1983. Strategies for multiattribute binary choice. Journal of Experimental Psychology: Learning, Memory and Cognition 9, 676-696.

Savage, L. J., 1954. The Foundations of Statistics. Wiley, New York. 
Scott, D., 1964. Measurement structures and linear inequalities. Journal of Mathematical Psychology 1, 233-247.

Scott, D., Suppes, P., 1958. Foundational aspects of theories of measurement. Journal of Symbolic Logic 23, 113-128.

Shapiro, L., 1979. Conditions for expected uility maximization. Annals of Statistics 7, 1288-1302.

Svenson, O., 1979. Process description of decision making. Organizational Behavior and Human Performance 23, 86-112.

Tsoukiàs, A., Perny, P., Vincke, P., 2002. From concordance/discordance to the modelling of positive and negative reasons in decision aiding. In: Aiding Decisions with Multiple Criteria: Essays in Honor of Bernard Roy. Kluwer, Dordrecht, pp. 147-174.

Tversky, A., 1969. Intransitivity of preferences. Psychological Review 76, 3148.

Vind, K., 1991. Independent preferences. Journal of Mathematical Economics 20, 119-135.

von Winterfeldt, D., Edwards, W., 1986. Decision analysis and behavioral research. Cambridge University Press, Cambridge.

Wakker, P. P., 1984. Cardinal coordinate independence for expected utility. Journal of Mathematical Psychology 28 (1), 110-117.

Wakker, P. P., 1988. Derived strength of preference relations on coordinates. Economic Letters 28, 301-306.

Wakker, P. P., 1989. Additive representations of preferences: A new foundation of decision analysis. Kluwer, Dordrecht.

Weymark, J. A., 1981. Generalized Gini inequality indices. Mathematical Social Sciences 1, 409-430. 